TRANSACTIONS OF THE

AMERICAN MATHEMATICAL SOCIETY

Volume 357, Number 1, Pages 309-335

S 0002-9947(04)03478-6

Article electronically published on April 27, 2004

\title{
GLAUBERMAN-WATANABE CORRESPONDING $p$-BLOCKS OF FINITE GROUPS WITH NORMAL DEFECT GROUPS ARE MORITA EQUIVALENT
}

\author{
MORTON E. HARRIS
}

\begin{abstract}
Let $G$ be a finite group and let $A$ be a solvable finite group that acts on $G$ such that the orders of $G$ and $A$ are relatively prime. Let $b$ be a $p$ block of $G$ with normal defect group $D$ such that $A$ stabilizes $b$ and $D \leq C_{G}(A)$. Then there is a Morita equivalence between the block $b$ and its Watanabe correspondent block $W(b)$ of $C_{G}(A)$ given by a bimodule $M$ with vertex $\Delta D$ and trivial source that on the character level induces the Glauberman correspondence (and which is an isotypy by a theorem of Watanabe).
\end{abstract}

\section{INTRODUCTION AND STATEMENTS}

This study was suggested by the work of S. Koshitani and G. Michler in [13].

The Theory of Blocks of Finite Groups was introduced and significantly developed by R. Brauer in [1] and 2]. Clearly Brauer's First Main Theorem (in [1]) underlines the importance of studying a block $B$ of a finite group $G$ with normal defect group D. In [16], W.F. Reynolds, using Clifford Theory, presented a deep analysis of the character theory of such a block $B$. In [15], B. Külshammer, using fundamental Clifford theoretic methods of E.C. Dade, showed that, in the context of a standard " $p$-modular system" $(\mathcal{K}, \mathcal{O}, k=\mathcal{O} / J(\mathcal{O}))$, the block algebra $\mathcal{A}$ over $\mathcal{O}$ of such a block $B$ is $\mathcal{O}$-algebra isomorphic to a full matrix algebra over a twisted group algebra $\mathcal{B}$ over $\mathcal{O}$ of the group $\mathcal{N}$ of [16]. In our main theorem (Theorem 2 ), we use the approach of [8] and [15] to demonstrate that the Morita equivalence between $\mathcal{A}$ and $\mathcal{B}$ is given by a bimodule with a diagonal vertex and trivial source. Moreover we are able to incorporate the Glauberman-Watanabe context into the analysis to demonstrate that there is such a Morita equivalence that gives the Glauberman correspondence on the character level. This analysis also extends the character theoretic analysis of W.F. Reynolds in [16].

Throughout this section, $G$ will denote a finite group and $A$ will denote a solvable finite group that acts on $G$ on the right and such that $(|G|,|A|)=1$. Let $C=C_{G}(A)$ and let $\operatorname{Irr}(G)$ and $\operatorname{Irr}(G)^{A}$ denote the sets of ordinary irreducible and $A$-invariant ordinary irreducible characters of $G$, respectively. In [6], G. Glauberman produced a bijection $\pi(G, A): \operatorname{Irr}(G)^{A} \rightarrow \operatorname{Irr}(C)$ satisfying natural basic properties (cf. [10] Theorem 13.1]).

Let $p$ be a prime and let $\mathcal{O}$ be a complete discrete valuation ring of characteristic zero such that $k=\mathcal{O} / J(\mathcal{O})$ is an algebraically closed field of prime characteristic

Received by the editors October 9, 2002 and, in revised form, July 29, 2003.

2000 Mathematics Subject Classification. Primary 20C20.

(C)2004 American Mathematical Society 
$p$ and such that if $\mathcal{K}$ is the fraction field of $\mathcal{O}$, then $(\mathcal{K}, \mathcal{O}, k)$ is "big enough" for all subgroups of $G \rtimes A$. As is standard, the natural ring epimorphism - : $\mathcal{O} \rightarrow$ $\mathcal{O} / J(\mathcal{O})=k$ induces a natural $\mathcal{O}$-algebra epimorphism $-: \mathcal{A} \rightarrow \overline{\mathcal{A}}=\mathcal{A} / J(\mathcal{O}) \mathcal{A}$ for any $\mathcal{O}$-algebra $\mathcal{A}$.

Let $B \ell(G)$ and $B \ell(G)^{A}$ denote the set of $p$-blocks of $G$ and of $A$-stable $p$-blocks of $G$, resp., in this context. Let $b \in B \ell(G)^{A}$ with defect group $D$ such that $D \leq C=$ $C_{G}(A)$. In [19, Theorems 1 and 2] A. Watanabe proved that then $\operatorname{Irr}(b) \subseteq \operatorname{Irr}(G)^{A}$ and $\{\pi(G, A)(\chi) \mid \chi \in \operatorname{Irr}(b)\}$ is the set of ordinary irreducible characters of a $p$-block of $C$ with defect group $D$ which we shall denote by $W(b)$. Moreover, she also proved that the Glauberman correspondence induces an isotypy between $b$ and $W(b)([19$, Theorem 2]).

Clearly $A$ acts on $N_{G}(D)$ and the Brauer correspondent $p$-block $B r_{D}(b)$ of $b$ is an $A$-stable $p$-block of $N_{G}(D)$ with defect group $D$. Also the Brauer correspondent $B r_{D}(W(b))$ is a $p$-block of $N_{C}(D)$ with defect group $D$ and $W\left(B r_{D}(b)\right)$ is also a $p$-block of $N_{C}(D)$ with defect group $D$.

For the convenience of the reader, we include a reformulation and an alternate proof of a result of S. Koshitani and G. Michler ([14, Theorem 2.12]) that links our main result presented below with the general Glauberman-Watanabe correspondence described above.

Theorem 1. $W\left(B r_{D}(b)\right)=B r_{D}(W(b))$.

For our main result, we also assume that the defect group $D$ of $b$ is normal in $G$ and hence normal in $C$. In the main result (Proposition 3.3) of [14, S. Koshitani and G. Michler demonstrated that, in this case, the $k$-algebras $k G \bar{b}$ and $k C \overline{W(b)}$ are Morita equivalent. The first part of our main result (Theorem 2), just below, stating that there is a Morita equivalence "over $\mathcal{O}$ " (that induces a Morita equivalence "over $k$ ") is essentially in the paper [14. In fact, S. Koshitani observed this fact in [13]. Moreover this "lifting from $k$ to $\mathcal{O}$ " is to be expected in view of the work of L. Puig for blocks with a normal defect group (cf. [18, Section 45 and Proposition 38.8]). Since "coefficients in $\mathcal{O}$ " provides the connection in finite group representation theory between the characteristic $p$ and classical characteristic 0 representation theories, such investigations are very important.

Our main result is:

Theorem 2. In this situation, there is a Morita equivalence between the block algebras $(\mathcal{O} G) b$ and $(\mathcal{O} C) W(b)$ given by an indecomposable $(\mathcal{O} G) b$-mod- $(\mathcal{O} C) W(b)$ bimodule $M$ with the following properties:

(i) when viewed as an $\mathcal{O}(G \times C)$-module, $M$ has $\Delta D=\{(u, u) \mid u \in D\}$ as a vertex and a trivial $\mathcal{O} \Delta D$-source; and

(ii) the bijection between the sets of ordinary irreducible characters $\operatorname{Irr}_{\mathcal{K}}(G, b)$ and $\operatorname{Irr}_{\mathcal{K}}(C, W(b))$ induced by the Morita equivalence given by $M$ is precisely the Glauberman correspondence.

Theorems 1 and 2 immediately yield:

Corollary 3. In the Glauberman-Watanabe context $\left(G, A, b \in B \ell(G)^{A}, C=\right.$ $\left.C_{G}(A), W(b), D \leq C\right)$, the Brauer correspondent blocks $\left(N_{G}(D), B r_{D}(b)\right)$ and $\left(N_{C}(D), B r_{D}(W(b))\right)$ are Morita equivalent with an equivalence given by an indecomposable $\mathcal{O} N_{G}(D) B r_{D}(b)$-mod- $\left(\mathcal{O} N_{C}(D) B r_{D}(W(b))\right.$ bimodule $M$ such that, when viewed as an $\mathcal{O}\left(N_{G}(D) \times N_{C}(D)\right)$-module, $M$ has $\Delta D=\{(u, u) \mid u \in D\}$ 
as a vertex and a trivial $\mathcal{O}(\Delta D)$-source. Moveover the bijection between the sets of ordinary irreducible characters $\operatorname{Irr}_{\mathcal{K}}\left(N_{G}(D), B r_{D}(b)\right)$ and $\operatorname{Irr}_{\mathcal{K}}\left(N_{C}(D), B r_{D}(W(b))\right.$ induced by the Morita equivalence is precisely the Glauberman correspondence.

Clearly the main result (Proposition 3.3) of [14 is a consequence of Theorem 2.

Our notation is standard and tends to follow the notation of [5, [9] and [10].

In particular, all rings are assumed to have identities.

Section 1 is comprised of a variety of results that are required for our proofs of Theorems 1 and 2 which are presented in Section 2.

The author thanks Everett C. Dade for his wise and helpful comments.

\section{Preliminary Results}

Let $m$ be a positive integer and let $Q$ be an abelian group. By definition, $Q$ is said to be $m$-divisible if for each $x \in Q$ there is a $y \in Q$ such that $y^{m}=x$, in which case $Q$ is also $n$-divisible whenever $n \mid m$.

The proof of [10, Lemma 11.14] is readily adapted to prove:

Lemma 1.1. Let $Q$ be a subgroup of an abelian group $M$ such that $|M / Q|$ is finite. Assume also that $Q$ is $|M / Q|$-divisible. Then $Q$ is complemented in $M$.

Let $M$ be an abelian group on which a finite group $G$ of order $m$ acts trivially.

The following results are well known and easy to verify.

Lemma 1.2. Let $c \in Z^{2}(G, M)$. Then

(a) $c(g, 1)=c(1,1)=c(1, g)$ for all $g \in G$;

(b) $c\left(g, g^{-1}\right)=c\left(g^{-1}, g\right)$ for all $g \in G$;

(c) if $c^{\prime}: G \times G \rightarrow M$ is defined by $c^{\prime}(g, h)=c(g, h) c(1,1)^{-1}$ for all $(g, h) \in G \times G$, then $c^{\prime} \in Z^{2}(G, M)$ and $c^{\prime}(g, 1)=c^{\prime}(1, g)=1$ for all $g \in G$;

(d) assume that $c(g, 1)=1=c(1, g)$ for all $g \in G$ and let $Z=\langle c(g, h)|(g, h) \in$ $G \times G\rangle$, so that $Z \leq M$. Let $\hat{G}=Z \times G$ and define a multiplication on $\hat{G}$ by

$$
\left(z_{1}, g_{1}\right)\left(z_{2}, g_{2}\right)=\left(z_{1} z_{2} c\left(g_{1}, g_{2}\right), g_{1} g_{2}\right)
$$

for all $z_{1}, z_{2} \in Z$ and all $g_{1}, g_{2} \in G$. Then $\hat{G}$ is a group with identity $(1,1)$ and $(z, g)^{-1}=\left(z^{-1} c\left(g, g^{-1}\right)^{-1}, g^{-1}\right)$ for all $z \in Z$ and $g \in G$. Also

$$
1 \rightarrow Z \stackrel{i}{\rightarrow} \hat{G} \stackrel{\pi_{1}}{\rightarrow} G \rightarrow 1
$$

is a short exact sequence of groups where $\pi_{1}$ is the first component projection and $i: Z \rightarrow G$ is defined by $z \mapsto(z, 1)$ for all $z \in Z$, so that $i(Z) \leq Z(\hat{G})$. Suppose also that the group $E$ acts on $G$ on the right and $c\left(g^{e}, h^{e}\right)=c(g, h)$ for all $g, h \in G$ and all $e \in E$. Let $E$ act trivially on the right on $Z$ and diagonally on $Z \times G$. Then $E$ acts on the group $\hat{G}=Z \times G$ and (1.1) is a short exact sequence of E-groups.

Remark 1.3. Suppose that $(\mathcal{K}, \mathcal{O}, k)$ is a $p$-modular system for the finite group $G$, that $G$ acts trivially on $\mathcal{O}^{\times}$, and that $c \in Z^{2}\left(G, \mathcal{O}^{\times}\right)$with $c(g, 1)=1=c(1, g)$ for all $g \in G$. Let $\mathcal{A}=\oplus_{g \in G} \mathcal{O} \tau_{g}$ be an associated twisted group $\mathcal{O}$-algebra over $G$, where $\tau_{g} \tau_{h}=c(g, h) \tau_{g h}$ for all $g, h \in G$. Then $1_{\mathcal{A}}=\tau_{1}$ and $\left(\tau_{g}\right)^{-1}=c\left(g, g^{-1}\right)^{-1} t_{g^{-1}}$ for all $g \in G$.

Suppose also that $Z=\langle c(g, h) \mid g, h \in G\rangle$ is a finite (and hence cyclic) subgroup of $\mathcal{O}^{\times}$of order $n$ with $(n, p)=1$. Let $\hat{G}=Z \times G$ be as above and let $\hat{e}=$ $\frac{1}{n} \sum_{z \in Z} z^{-1}(z, 1) \in \mathcal{O} i(Z) \leq Z(\mathcal{O} \hat{G})$. Then $\hat{e}$ is a block idempotent of $\mathcal{O} i(Z)$ and is central in $\mathcal{O} \hat{G}$. Also $\mathcal{O} \hat{G}$ is a $G$-crossed product $\mathcal{O}$-algebra with $(\mathcal{O} \hat{G})_{g}=$ 
$(\mathcal{O} i(Z))(1, g)$ for all $g \in G$ and $(z, g) \hat{e}=z(1, g) \hat{e}$ for all $z \in Z$ and all $g \in G$. Hence $(\mathcal{O} \hat{G}) \hat{e}=\oplus_{g \in G} \mathcal{O}(1, g) \hat{e}$ in $\mathcal{O}$-mod and the $\mathcal{O}$ linear map $\alpha:(\mathcal{O} \hat{G}) \hat{e} \rightarrow \mathcal{A}$ such that $(1, g) \hat{e} \mapsto \tau_{g}$ for all $g \in G$ is an $\mathcal{O}$-algebra isomorphism. Here $(\mathcal{O} \hat{G}) \hat{e}$ is the direct sum of the block algebras of $\hat{G}$ that cover the block $\hat{e}$ of $\mathcal{O} i(Z)$.

As above, let the finite group $G$ of order $m$ act trivially on the abelian group $M$. Assume also that $M$ is $m$-divisible and that $\Omega_{m}(M)=\left\{x \in M \mid x^{m}=1\right\}$ is finite.

Let $U=\left\{c \in Z^{2}(G, M) \mid c^{m}=1\right\}$, so that $U$ is a finite subgroup of $Z^{2}(G, M)$. Note that $H^{2}(G, M)$ has exponent dividing $m=|G|$ (9, I, Satz 16.19]) and that $B^{2}(G, M)$ is $m$-divisible.

The proof of [10, Theorem 11.15] yields:

Lemma 1.4. Under these conditions, there is a subgroup $W \leq U$ such that $Z^{2}(G, M)$ $=B^{2}(G, M) \times W$ and hence $H^{2}(G, M) \cong W$.

Let $R$ be a commutative ring.

Lemma 1.5. Let $\mathcal{A}$ be an $R$-algebra and let $\mathcal{S}$ be an $R$-subalgebra of $\mathcal{A}$ such that $\mathcal{S} \cong M_{r}(R)$ as $R$-algebras, where $M_{r}(R)$ is the $R$-algebra of all $r \times r$ matrices over $R$ for some positive integer $r$. Then:

(a) $C_{\mathcal{A}}\left(\mathcal{S}^{\times}\right)=C_{\mathcal{A}}(\mathcal{S})$; and

(b) $N_{\mathcal{A}^{\times}}\left(\mathcal{S}^{\times}\right)=N_{\mathcal{A}^{\times}}(\mathcal{S})$.

Proof. Clearly $C_{\mathcal{A}}(\mathcal{S}) \subseteq C_{\mathcal{A}}\left(\mathcal{S}^{\times}\right)$and $N_{\mathcal{A}^{\times}}(\mathcal{S}) \subseteq N_{\mathcal{A}^{\times}}\left(\mathcal{S}^{\times}\right)$since $1_{\mathcal{S}}^{\alpha}=1_{\mathcal{S}} \in \mathcal{S}^{\times}$for all $\alpha \in N_{\mathcal{A} \times}(\mathcal{S})$. If $r=1$, then $\mathcal{S}=R 1_{\mathcal{S}}$. Hence $C_{\mathcal{A}}\left(\mathcal{S}^{\times}\right) \subseteq C_{\mathcal{A}}(\mathcal{S}), N_{\mathcal{A} \times}\left(\mathcal{S}^{\times}\right) \subseteq$ $N_{\mathcal{A}^{\times}}(\mathcal{S})$ and we are done. Assume that $r \geq 2$. Clearly if $x \in \mathcal{S}$, then $x \in \mathcal{S}^{\times}$if and only if $\operatorname{det}(x) \in R^{\times}$. Also $\mathcal{S}$ has an $R$-basis $\left\{E_{i j} \mid 1 \leq i, j \leq r\right\}$ such that

$$
E_{i j} E_{m n}= \begin{cases}0 & \text { if } j \neq m, \\ E_{i n} & \text { if } j=m .\end{cases}
$$

Let $\alpha \in C_{\mathcal{A}}\left(\mathcal{S}^{\times}\right)$. Then $\alpha 1_{\mathcal{S}}=1_{\mathcal{S}} \alpha$ and $\alpha\left(1_{\mathcal{S}}+E_{i j}\right)=\left(1_{\mathcal{S}}+E_{i j}\right) \alpha$ for all $1 \leq i, j \leq r$ with $i \neq j$. Thus $\alpha E_{i j}=E_{i j} \alpha$ and $\alpha E_{i i}=\alpha E_{i j} E_{j i}=E_{i j} E_{j i} \alpha=E_{i i} \alpha$ for all $1 \leq i, j \leq r$ with $i \neq j$. Consequently (a) holds. A similar argument demonstrates that if $\alpha \in N_{\mathcal{A}^{\times}}\left(\mathcal{S}^{\times}\right)$, then $1_{\mathcal{S}}^{\alpha}=1_{\mathcal{S}},\left(1_{\mathcal{S}}+E_{i j}\right)^{\alpha}=1_{\mathcal{S}}+E_{i j}^{\alpha}$ and $E_{i j}^{\alpha} \in \mathcal{S}$ for all $1 \leq i, j \leq r$ with $i \neq j$. Thus $E_{i i}^{\alpha}=\left(E_{i j} E_{j i}\right)^{\alpha}=E_{i j}^{\alpha} E_{j i}^{\alpha} \in \mathcal{S}$ for all $1 \leq i, j \leq r$ with $i \neq j, \mathcal{S}^{\alpha} \subseteq \mathcal{S}$ and $\mathcal{S}^{\alpha^{-1}} \subseteq \mathcal{S}$ and hence $\mathcal{S}^{\alpha}=\mathcal{S}$ and (b) follows.

Let $\mathcal{A}=\oplus_{g \in G} \mathcal{A}_{g}$ be a $G$-graded $R$-algebra (cf. [4]). For each $X \subseteq G$, let $\mathcal{A}_{X}=\oplus_{x \in X} \mathcal{A}_{x}$, so that if $H \leq G$, then $\mathcal{A}_{H}$ is an $H$-graded $R$-subalgebra of $\mathcal{A}$ since $1_{\mathcal{A}} \in \mathcal{A}_{1}$.

Lemma 1.6. Let $H \leq G$, let $b$ be an idempotent of $\mathcal{A}_{H}$ and let $\alpha \in b \mathcal{A}_{H} b$. Suppose that there is an element $\beta \in \mathcal{A}$ such that $\alpha \beta=b$ and $\beta \alpha=b$ (so that $\alpha(b \beta b)=b$ and $(b \beta b) \alpha=b)$. Then $b \beta b \in b \mathcal{A}_{H} b$.

Proof. First suppose that $b=1_{\mathcal{A}}$ and let $\mathcal{T}$ be a right transversal of $H$ in $G$ with $1 \in \mathcal{T}$ so that $G=\bigcup_{t \in \mathcal{T}} H t$, where the union is disjoint. Let $\beta=\sum_{\substack{h \in H \\ t \in \mathcal{T}}} b_{h t}$, where $b_{h t} \in \mathcal{A}_{h t}$ for all $h \in H$ and $t \in \mathcal{T}$. Then $1_{\mathcal{A}}=\sum_{t \in \mathcal{T}}\left(\alpha\left(\sum_{h \in H} b_{h t}\right)\right) \in \mathcal{A}_{1}$, where $\alpha\left(\sum_{h \in H} b_{h t}\right) \in \mathcal{A}_{H t}$ for all $t \in \mathcal{T}$. Thus if $t \neq 1$, then $\alpha\left(\sum_{h \in H} b_{h t}\right)=0$ and so $\left(\sum_{h \in H} b_{h t}\right)=0$ since $\alpha$ is a unit. Consequently $\beta \in \mathcal{A}_{H}$. For the general case, set $\alpha^{*}=\alpha+\left(1_{\mathcal{A}}-b\right)$ and $\beta^{*}=b \beta b+\left(1_{\mathcal{A}}-b\right)$. Then $\alpha^{*} \beta^{*}=1_{\mathcal{A}}=\beta^{*} \alpha^{*}$, where $\alpha^{*} \in \mathcal{A}_{H}$. By the case above, we conclude that $\beta^{*}=b \beta b+\left(1_{\mathcal{A}}-b\right) \in \mathcal{A}_{H}$ and hence $b \beta b \in b \mathcal{A}_{H} b$ and we are done. 
Let $\mathcal{A}=\oplus_{g \in G} \mathcal{A}_{g}$ be a $G$-graded crossed-product $R$-algebra (with an identity $\left.1_{\mathcal{A}} \in \mathcal{A}_{1}\right)$ and assume that $\mathcal{A}_{1}$ is commutative. Thus $\mathcal{A}_{g} \cap \mathcal{A}^{\times}$is nonempty for each $g \in G$. Here we have the short exact sequence of groups

$$
1 \rightarrow \mathcal{A}_{1}^{\times} \rightarrow \mathcal{G} r\left(\mathcal{A}^{\times}\right) \stackrel{\operatorname{deg}}{\rightarrow} G \rightarrow 1
$$

and $G$ acts on $\mathcal{A}_{1}$ (on the right) as defined by

$$
\alpha^{g}=\alpha^{u_{g}}=u_{g}^{-1} \alpha u_{g} \text { for all } \alpha \in \mathcal{A}_{1}
$$

for some (and hence every) $u_{g} \in \mathcal{A}_{g} \cap \mathcal{A}^{\times}$for each $g \in G$.

For each $g \in G$, choose $u_{g} \in \mathcal{A}_{g} \cap \mathcal{A}^{\times}$. Here $\mathcal{A}_{g}=\mathcal{A}_{1} u_{g}=u_{g} \mathcal{A}_{1}$ and $u_{g} u_{h}=$ $u_{g h} c(g, h)$ for a unique $c(g, h) \in \mathcal{A}_{1}^{\times}$for all $g, h \in G$ and $c \in Z^{2}\left(G, \mathcal{A}_{1}^{\times}\right)$. Clearly multiplication in $\mathcal{A}$ is determined by $\mathcal{A}_{1}$, the action of $G$ on $\mathcal{A}_{1}$ and the element $c \in Z^{2}\left(G, \mathcal{A}_{1}^{\times}\right)$. Also, the element $c B^{2}\left(G, \mathcal{A}_{1}^{\times}\right)$is independent of the choices of the elements $u_{g} \in \mathcal{A}_{g} \cap \mathcal{A}^{\times}$for all $g \in G$.

Let $\mathcal{B}=\oplus_{g \in G} \mathcal{B}_{g}$ also be a $G$-graded crossed product $R$-algebra (with an identity $1_{\mathcal{B}} \in \mathcal{B}_{1}$ ) and let $\sigma: \mathcal{A}_{1} \rightarrow \mathcal{B}_{1}$ be an $R$-algebra isomorphism (so that $\mathcal{B}_{1}$ is commutative) such that $\sigma$ commutes with the action of $G$ on $\mathcal{A}_{1}$ and on $\mathcal{B}_{1}$ (i.e., $\sigma\left(\alpha^{g}\right)=\sigma(\alpha)^{g}$ for all $\alpha \in \mathcal{A}_{1}$ and all $\left.g \in G\right)$. Let $s: H^{2}\left(G, \mathcal{A}_{1}^{\times}\right) \rightarrow H^{2}\left(G, \mathcal{B}_{1}^{\times}\right)$be the group isomorphism induced by $\sigma$.

The following result is easy to prove and well known:

Proposition 1.7. There is a $G$-graded $R$-algebra isomorphism $\tilde{\sigma}: \mathcal{A} \rightarrow \mathcal{B}$ that extends $\sigma$ if and only if $s\left(c B^{2}\left(G, \mathcal{A}_{1}^{\times}\right)\right)$is the element of $H^{2}\left(G, \mathcal{B}_{1}^{\times}\right)$determined by the action of $G$ on $\mathcal{B}_{1}$ via $\mathcal{B}$.

As in the Introduction, we let $\mathcal{O}$ be a complete discrete valuation ring of characteristic zero such that $k=\mathcal{O} / J(\mathcal{O})$ is an algebraically closed field of prime characteristic $p$. Let $\mathcal{K}$ denote the field of fractions of $\mathcal{O}$. Such rings exist (cf. [17, II, Theorem 3]) and satisfy [18, assumption (2.1)]. Moreover by [17] II, Proposition 8]:

(1.2) there is a multiplicative injection $f: k \rightarrow \mathcal{O}$ such that

(a) $f(y)+J(\mathcal{O})=y$ for all $y \in k=\mathcal{O} / J(\mathcal{O})$; and

(b) if $x \in \mathcal{O}$, then $x \in f(k)$ if and only if $x$ is a $p^{n}$-power in $\mathcal{O}$ for every integer $n \geq 0$.

Thus $f\left(1_{k}\right)=1_{\mathcal{O}}, f\left(k^{\times}\right) \leq \mathcal{O}^{\times}, \mathcal{O}^{\times}=f\left(k^{\times}\right) \times(1+J(\mathcal{O}))$, any element of $\mathcal{O}^{\times}$ of finite order prime to $p$ lies in $f\left(k^{\times}\right)$(cf. [18, Lemma 2.3]) and $f: k^{\times} \rightarrow f\left(k^{\times}\right)$ is a group isomorphism.

For the remainder of this section, we shall also assume that every $\mathcal{O}$-algebra $\mathcal{A}$ is finitely generated in $\mathcal{O}$-mod. Consequently $\mathcal{A} / J(\mathcal{A})$ is a finite-dimensional split semi-simple $k$-algebra. We denote the unity element of $\mathcal{A}$ by $1_{\mathcal{A}}$ (or sometimes simply by 1 ). Also we shall assume that every $\mathcal{A}$-module is a finitely generated and unitary $\mathcal{A}$-module. Note that every free $\mathcal{O}$-module is a torsion free $\mathcal{O}$-module.

If $r$ is a positive integer, then $M_{r}(k)$ and $M_{r}(\mathcal{O})$ will denote the $\mathcal{O}$-algebras of all $r \times r$ matrices over $k$ and $\mathcal{O}$, respectively. As in [18, Section 7], an $\mathcal{O}$-algebra $\mathcal{S}$ is called $\mathcal{O}$-simple if $\mathcal{S}$ is $\mathcal{O}$-algebra isomorphic to some $M_{r}(\mathcal{O})$ and $\mathcal{S}$ is said to be $\mathcal{O}$-semi-simple if $\mathcal{S}$ is $\mathcal{O}$-algebra isomorphic to a direct sum of $\mathcal{O}$-simple $\mathcal{O}$-algebras.

Let $\mathcal{A}$ be an $\mathcal{O}$-algebra and let $m$ be a positive integer with $(m, p)=1$. Let $\mu: \mathcal{A} \rightarrow \mathcal{A}$ be the function such that $x \mapsto x^{m}$ for all $x \in \mathcal{A}$.

Proposition 1.8. $\mu$ induces a bijection of $1_{\mathcal{A}}+J(\mathcal{A})$ onto itself. 
To prove this, we set $J=J(\mathcal{A})$ and note the following two trivialities.

Lemma 1.9. Let $\mathcal{G}$ be a not necessarily finite group and let $x, y \in \mathcal{G}$ be such that $x^{m}=y$ and $|x| \mid p^{k}$ for some positive integer $k \geq 1$. Then $x=y^{a}$ for any $a, b \in Z$ such that $1=m a+p^{k} b$. Hence if $x_{1}, x_{2}$ are $p$-elements of $\mathcal{G}$ such that $x_{1}^{m}=y=x_{2}^{m}$, then $x_{1}=x_{2}$.

Lemma 1.10. Let $n, k$ be positive integers. Then:

(a) $\left(J^{n} / J^{n+1},+\right)$ is an abelian group of exponent $p$;

(b) the map $J^{n} \mapsto 1_{\mathcal{A}}+J^{n}$ such that $a \mapsto 1_{\mathcal{A}}+a$ for all $a \in J^{n}$ induces a group isomorphism of $\left(J^{n} / J^{n+1},+\right)$ onto the multiplicative group $\left(1_{\mathcal{A}}+J^{n}\right) /\left(1_{\mathcal{A}}+J^{n+1}\right)$; and

(c) the multiplicative group $\left(1_{\mathcal{A}}+J^{n}\right) /\left(1_{\mathcal{A}}+J^{n+k}\right)$ has exponent dividing $p^{k}$.

Proof of Proposition 1.8. By [18, Lemma 45.5], we have

$$
1_{\mathcal{A}}+J=\lim _{\longleftarrow}\left(\left(1_{\mathcal{A}}+J\right) /\left(1_{\mathcal{A}}+J^{n}\right)\right) .
$$

For each integer $k \geq 1$, choose $a_{k}, b_{k} \in Z$ such that $m a_{k}+p^{k} b_{k}=1$. Assume that $a_{0}=0$ and $b_{0}=1$. Let $y \in 1_{\mathcal{A}}+J$. If $k \geq 1$, then $y^{a_{k}}\left(1+J^{k+1}\right)$ is the unique element $w$ of $\left(1_{\mathcal{A}}+J\right) /\left(1_{\mathcal{A}}+J^{k+1}\right)$ such that $w^{m}=y\left(1_{\mathcal{A}}+J^{k+1}\right)$ by Lemmas 1.9 and 1.10 (c). Moreover, $\left(y^{a_{k}}\left(1_{\mathcal{A}}+J^{k}\right)\right)^{m}=y\left(1_{\mathcal{A}}+J^{k}\right)$ so that $y^{a_{k}}\left(1_{\mathcal{A}}+J^{k}\right)=$ $y^{a_{k-1}}\left(1_{\mathcal{A}}+J^{k}\right)$. Thus $u=\left(y\left(1_{\mathcal{A}}+J\right), y^{a_{1}}\left(1_{\mathcal{A}}+J^{2}\right), y^{a_{2}}\left(1_{\mathcal{A}}+J^{3}\right), \ldots\right)$ is an element of $\lim _{\longleftarrow}\left(\left(1_{\mathcal{A}}+J\right) /\left(1_{\mathcal{A}}+J^{n}\right)\right)$ such that

$$
u^{m}=\left(y\left(1_{\mathcal{A}}+J\right), y\left(1_{\mathcal{A}}+J^{2}\right), y\left(1_{\mathcal{A}}+J^{3}\right), \ldots\right)=y .
$$

Assume that $z=\left(z_{0}\left(1_{\mathcal{A}}+J\right), z_{1}\left(1_{\mathcal{A}}+J^{2}\right), z_{2}\left(1_{\mathcal{A}}+J^{3}\right), \ldots\right)$ is an element of

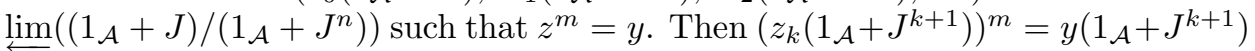
and Lemmas 1.9 and 1.10(c) imply that

$$
z_{k}\left(1_{\mathcal{A}}+J^{k+1}\right)=y^{a_{k}}\left(1_{\mathcal{A}}+J^{k+1}\right)
$$

for all $k \geq 0$. Thus $z=u$ and we are done.

We continue with this situation. We shall require the following extension of [18, Lemma 45.6].

Let $X$ be a group containing $1_{\mathcal{A}}+J(\mathcal{A})$ as a normal subgroup. Assume also that the subgroup $1_{\mathcal{A}}+\left(J(\mathcal{A})^{n}\right)$ is normal in $X$ for every integer $n \geq 1$ and that $E$ is a finite subgroup of $X$ with $|E|=m$ relatively prime to $p$ such that $X=$ $\left(1_{\mathcal{A}}+J(\mathcal{A})\right) E$. Thus $E \cap\left(1_{\mathcal{A}}+J(\mathcal{A})\right)=1_{\mathcal{A}}$ by Proposition 1.8 .

Lemma 1.11. Let $e \in E$ and let $x \in\left(1_{\mathcal{A}}+J(\mathcal{A})\right)$ e be of order prime to $p$. Then there is an element $j \in J(\mathcal{A})$ such that $x^{1_{\mathcal{A}}+j}=e$.

Proof. Clearly we may assume that $X=\left(1_{\mathcal{A}}+J(\mathcal{A})\right)\langle e\rangle$. Let $\alpha=|x|$ and $\beta=|e|$. Then $x^{\alpha} \in 1_{\mathcal{A}}+J(\mathcal{A})$ and hence $\alpha=\beta s$ for some positive integer $s$. Here $x^{\beta} \in 1_{\mathcal{A}}+$ $J(\mathcal{A})$ and $\left(x^{\beta}\right)^{s}=1_{\mathcal{A}}$. Since $(\alpha, p)=1$, we conclude that $(s, p)=1$. Thus $x^{\beta}=1_{\mathcal{A}}$ because of Proposition 1.8. Consequently $\alpha=\beta$. Since $X=\left(1_{\mathcal{A}}+J(\mathcal{A})\right)\langle x\rangle$ and $\langle x\rangle \cap\left(1_{\mathcal{A}}+J(\mathcal{A})\right)=1_{\mathcal{A}},[18$ Lemma 45.6] yields an element $j \in J(\mathcal{A})$ such that $\langle x\rangle^{1_{\mathcal{A}}+j}=\langle e\rangle$. Then $x^{1_{\mathcal{A}}+j} \in\langle e\rangle \cap((1+J(\mathcal{A})) e)=\{e\}$ and we are done.

Let $\mathcal{A}$ be an $\mathcal{O}$-free $\mathcal{O}$-algebra. Then, as in [18, Theorem 7.3], there is an $\mathcal{O}$ semi-simple $\mathcal{O}$-subalgebra $\mathcal{S}$ such that $\mathcal{A}=\mathcal{S}+J(\mathcal{A})$ and, in fact, $\mathcal{S}$ is a maximal $\mathcal{O}$-semi-simple $\mathcal{O}$-subalgebra of $\mathcal{A}$. We require the following observations in this context. 
Proposition 1.12. (a) $J(\mathcal{A}) \cap \mathcal{S}=J(\mathcal{O}) \mathcal{S}=J(\mathcal{S})$ and $\mathcal{S} /(J(\mathcal{O}) \mathcal{S})$ is a k-semisimple $k$-algebra;

(b) $1_{\mathcal{A}}=1_{\mathcal{S}}$ and $\mathcal{S} \cap\left(1_{\mathcal{A}}+J(\mathcal{A})\right)=\mathcal{S}^{\times} \cap\left(1_{\mathcal{A}}+J(\mathcal{A})\right)=1_{\mathcal{A}}+J(\mathcal{S})$;

(c) $\mathcal{A}^{\times}=\left(1_{\mathcal{A}}+J(\mathcal{A})\right) \mathcal{S}^{\times}=\mathcal{S}^{\times}\left(1_{\mathcal{A}}+J(\mathcal{A})\right)$; and

(d) any two maximal $\mathcal{O}$-semi-simple $\mathcal{O}$-subalgebras of $\mathcal{A}$ are conjugate by an element of $1_{\mathcal{A}}+J(\mathcal{A})$.

(e) Assume also that $\mathcal{S}$ is $\mathcal{O}$-simple. Then $C_{\mathcal{A}}(\mathcal{S})=C_{\mathcal{A}}\left(\mathcal{S}^{\times}\right), N_{\mathcal{A}^{\times}}\left(\mathcal{S}^{\times}\right)=$ $N_{\mathcal{A} \times}(\mathcal{S})=\mathcal{S}^{\times} N_{1_{\mathcal{A}}+J(\mathcal{A})}(\mathcal{S})=N_{1_{\mathcal{A}}+J(\mathcal{A})}(\mathcal{S}) \mathcal{S}^{\times}$and $\left[\mathcal{S}^{\times}, N_{1_{\mathcal{A}}+J(\mathcal{A})}\left(\mathcal{S}^{\times}\right)\right] \leq \mathcal{S}^{\times} \cap$ $\left(1_{\mathcal{A}}+J(\mathcal{A})\right)=1_{\mathcal{A}}+J(\mathcal{S})$.

Proof. Clearly (a) holds and, since $1_{\mathcal{A}}-1_{\mathcal{S}}$ is an idempotent in $J(\mathcal{A})$, (b) also follows. Clearly $\left(1_{\mathcal{A}}+J(\mathcal{A})\right) \mathcal{S}^{\times} \leq \mathcal{A}^{\times}$. Let $s \in \mathcal{S}$ and $j \in J(\mathcal{A})$ be such that $s+j \in \mathcal{A}^{\times}$. Then there is an element $t \in \mathcal{S}$ such that $s t-1_{\mathcal{A}} \in J(\mathcal{A}) \cap \mathcal{S}=J(\mathcal{S})$ and $t s-1_{\mathcal{A}} \in J(\mathcal{A}) \cap \mathcal{S}=J(\mathcal{S})$. Thus $s t \in 1_{\mathcal{A}}+J(\mathcal{S})$ and $t s \in 1_{\mathcal{A}}+J(\mathcal{S})$ so that $s$ has an inverse $s^{-1}$ in $\mathcal{S}$. Then $s+j=s\left(1_{\mathcal{A}}+s^{-1} j\right) \in \mathcal{S}^{\times}(1+J(\mathcal{A}))$, (c) holds and (d) follows from [18, Theorem 7.3(c)]. Finally Lemma 1.5, (b) and (c) and the fact that $1+J(\mathcal{A}) \leq \mathcal{A}^{\times}$yield $(\mathrm{e})$.

Note that we always assume that any finite group $G$ acts trivially on $\mathcal{O}$.

Lemma 1.13. Let $\mathcal{A}$ be an $\mathcal{O}$-free $G$-algebra such that $\mathcal{A}=\mathcal{O} 1_{\mathcal{A}}+J(\mathcal{A})$. Then:

(a) $\mathcal{A}^{\times}=f\left(k^{\times}\right) 1_{\mathcal{A}} \times\left(1_{\mathcal{A}}+J(\mathcal{A})\right)$ as a $G$-group (since $G$ acts trivially on $\mathcal{O} 1_{\mathcal{A}}$ by hypothesis); and

(b) if $|G|=m$ is prime to $p$ and $\mathcal{A}$ is also commutative, then $f: k^{\times} \rightarrow \mathcal{O}^{\times}$ of (1.2) induces a natural group isomorphism $f^{*}: k^{\times} \rightarrow f\left(k^{\times}\right) 1_{\mathcal{A}}$ such that $x \mapsto f(x) 1_{\mathcal{A}}$ for all $x \in k^{\times}$and $f^{*}$ induces a group isomorphism $H^{2}\left(G, f^{*}\right)$ : $H^{2}\left(G, k^{\times}\right) \rightarrow H^{2}\left(G, \mathcal{A}^{\times}\right)$.

Proof. Since $\mathcal{O}^{\times} 1_{\mathcal{A}}=f\left(k^{\times}\right) 1_{\mathcal{A}} \times(1+J(\mathcal{O})) 1_{\mathcal{A}}$ and $\mathcal{A}^{\times}=\left(\mathcal{O}^{\times} 1_{\mathcal{A}}\right)\left(1_{\mathcal{A}}+J(\mathcal{A})\right)$, we have $\mathcal{A}^{\times}=\left(f\left(k^{\times}\right) 1_{\mathcal{A}}\right)\left(1_{\mathcal{A}}+J(\mathcal{A})\right)$. Let $x \in\left(f\left(k^{\times}\right) 1_{\mathcal{A}}\right) \cap\left(1_{\mathcal{A}}+J(\mathcal{A})\right)$. Then $x=$ $\alpha 1_{\mathcal{A}}=1_{\mathcal{A}}+j$, where $\alpha \in f\left(k^{\times}\right)$and $j \in J(\mathcal{A})$. Thus $(\alpha-1) 1_{\mathcal{A}} \in J(\mathcal{A}) \cap\left(\mathcal{O} 1_{\mathcal{A}}\right)=$ $J(\mathcal{O}) 1_{\mathcal{A}}, \alpha=1$ and $x=1_{\mathcal{A}}$ which proves (a). In the situation of (b), we have $H^{2}\left(G, \mathcal{A}^{\times}\right)=H^{2}\left(G, f\left(k^{\times}\right) 1_{\mathcal{A}}\right) \times H^{2}\left(G, 1_{\mathcal{A}}+J(\mathcal{A})\right)$ since the direct decomposition of (a) is a $G$-decomposition. As $m=|G|$ is relatively prime to $p$ and the $m$-th power map is an automorphism of $1_{\mathcal{A}}+J(\mathcal{A})$ by Proposition 1.8, we have

$$
H^{2}\left(G, 1_{\mathcal{A}}+J(\mathcal{A})\right)=1
$$

(by [9, I, Satz 16.19(a)]). Our proof is complete.

Let $D$ be a finite $p$-group and set $\mathcal{A}=\mathcal{O} D$. Let $\mathcal{I}(\mathcal{O} D)=\sum_{d \in D^{*}} \mathcal{O}(d-1)$ be the augmentation ideal of $\mathcal{A}$, so that $\mathcal{I}(\mathcal{O} D) \leq J(\mathcal{A}), \mathcal{A}=\mathcal{O} 1_{\mathcal{A}} \oplus \mathcal{I}(\mathcal{O} D)$ in $\mathcal{O}$-mod and $\mathcal{O} / \mathcal{I}(\mathcal{O} D) \tilde{=}$ as $\mathcal{O}$-algebras.

Lemma 1.14. (a)

$$
\mathcal{A}^{\times}=\left(\mathcal{O}^{\times} 1_{\mathcal{A}}\right) \times\left(1_{\mathcal{A}}+\mathcal{I}(\mathcal{O} D)\right)
$$

and

$$
1_{\mathcal{A}}+J(\mathcal{O} D)=\left(1_{\mathcal{A}}+J(\mathcal{O}) 1_{\mathcal{A}}\right) \times\left(1_{\mathcal{A}}+\mathcal{I}(\mathcal{O} D)\right)
$$

as groups and $\left(\mathcal{O}^{\times} 1_{\mathcal{A}}\right) \cap\left(1_{\mathcal{A}}+J(\mathcal{O} D)\right)=1_{\mathcal{A}}+J(\mathcal{O}) 1_{\mathcal{A}}$; and

(b) let $m$ be a positive integer with $(m, p)=1$ and let $\mu: 1_{\mathcal{A}}+J(\mathcal{A}) \rightarrow 1_{\mathcal{A}}+J(\mathcal{A})$ be the $m$-th power bijective map of Proposition 1.8. Then the restriction of $\mu$ to $1_{\mathcal{A}}+\mathcal{I}(\mathcal{O D}) \rightarrow 1_{\mathcal{A}}+\mathcal{I}(\mathcal{O} D)$ is a bijection. 
Proof. Let $x \in \mathcal{A}^{\times}$. Then $x=a 1_{\mathcal{A}}+i$, where $i \in \mathcal{I}(\mathcal{O} D)$ and $a \in \mathcal{O}$. Since $\mathcal{A} / \mathcal{I}(\mathcal{O} D) \tilde{=} \mathcal{O}, a \in \mathcal{O}^{\times}$and $x=\left(a 1_{\mathcal{A}}\right)\left(1_{\mathcal{A}}+a^{-1} i\right) \in\left(\mathcal{O}^{\times} 1_{\mathcal{A}}\right)\left(1_{\mathcal{A}}+\mathcal{I}(\mathcal{O} D)\right)$. Let $b \in \mathcal{O}^{\times}$be such that $b 1_{\mathcal{A}}=1_{\mathcal{A}}+i$, where $i \in \mathcal{I}(\mathcal{O} D)$. Then $(b-1) 1_{\mathcal{A}}=i=0, b=1$ and $\mathcal{A}^{\times}=\left(\mathcal{O}^{\times} 1_{\mathcal{A}}\right) \times\left(1_{\mathcal{A}}+\mathcal{I}(\mathcal{O} D)\right)$ as groups. Since $1_{\mathcal{A}}+\mathcal{I}(\mathcal{O} D) \leq 1_{\mathcal{A}}+J(\mathcal{O} D)$, $1_{\mathcal{A}}+J(\mathcal{O} D)=\left((1+J(\mathcal{O} D)) \cap\left(\mathcal{O}^{\times} 1_{\mathcal{A}}\right)\right) \times\left(1_{\mathcal{A}}+\mathcal{I}(\mathcal{O} D)\right)$. Let $a \in \mathcal{O}^{\times}$be such that $a 1_{\mathcal{A}}=1_{\mathcal{A}}+j$, where $j \in J(\mathcal{O} D)$. Then $(a-1) 1_{\mathcal{A}}=j \in J(\mathcal{O} D) \cap\left(\mathcal{O} 1_{\mathcal{A}}\right)=J(\mathcal{O}) 1_{\mathcal{A}}$ and (a) holds.

For (b), it suffices to prove that $\mu: 1_{\mathcal{A}}+\mathcal{I}(\mathcal{O} D) \rightarrow 1_{\mathcal{A}}+\mathcal{I}(\mathcal{O} D)$ is surjective. Let $i \in \mathcal{I}(\mathcal{O} D)$. Then $1_{\mathcal{A}}+i=\left(1_{\mathcal{A}}+j\right)^{m}$ for some $j \in J(\mathcal{O} D)$ by Proposition 1.8. Also $1_{\mathcal{A}}+j=a 1_{\mathcal{A}}+s$ for some $a \in \mathcal{O}$ and some $s \in \mathcal{I}(\mathcal{O} D)$. As $\mathcal{A} / \mathcal{I}(\mathcal{O} D) \cong \mathcal{O}$ as rings, $a^{m}=1$. Thus $1_{\mathcal{A}}+j=a 1_{\mathcal{A}}\left(1_{\mathcal{A}}+a^{-1} s\right)$, where $1_{\mathcal{A}}+a^{-1} s \in 1_{\mathcal{A}}+J(\mathcal{A})$. Now Lemma 1.13(a) implies that $a=1, j=s$ and we are done.

Next assume that $\mathcal{A}$ is a $G$-graded crossed-product $\mathcal{O}$-algebra that is $\mathcal{O}$-free. Note that $1_{\mathcal{A}} \in \mathcal{A}_{1}$.

Proposition 1.15. Assume that $\mathcal{A}_{1} / J\left(\mathcal{A}_{1}\right) \cong M_{r}(k)$ for some positive integer r. Let $\mathcal{S}$ be a maximal $\mathcal{O}$-semisimple subalgebra of $\mathcal{A}_{1}$ so that $1_{\mathcal{A}} \in \mathcal{S}$ and let $u_{g} \in \mathcal{A}_{g} \cap \mathcal{A}^{\times}$for each $g \in G$. Then:

(a) $\mathcal{S} \cong M_{r}(\mathcal{O})$ as $\mathcal{O}$-algebras, $\mathcal{A}_{1}=\mathcal{S}+J\left(\mathcal{A}_{1}\right), \mathcal{S} \cap J\left(\mathcal{A}_{1}\right)=J(\mathcal{O}) \mathcal{S}=J(\mathcal{S})$ and $\mathcal{A}_{1}^{\times}=\mathcal{S}^{\times}\left(1_{\mathcal{A}}+J\left(\mathcal{A}_{1}\right)\right)=\left(1_{\mathcal{A}}+J\left(\mathcal{A}_{1}\right)\right) \mathcal{S}^{\times}$

(b) for each $g \in G$, there is an element $w_{g} \in \mathcal{A}_{1}^{\times}$such that $v_{g}=w_{g} u_{g} \in$ $C_{\mathcal{A}_{g}}(\mathcal{S}) \cap \mathcal{A}^{\times}$

(c) $C_{\mathcal{A}}(\mathcal{S})=\oplus_{g \in G}\left(C_{\mathcal{A}_{1}}(\mathcal{S}) v_{g}\right)$ is a $G$-graded crossed-product $\mathcal{O}$-subalgebra of $\mathcal{A}=\oplus_{g \in G} \mathcal{A}_{g}$, where $\left(C_{\mathcal{A}}(\mathcal{S})\right)_{g}=C_{\mathcal{A}_{1}}(\mathcal{S}) v_{g}$ for all $g \in G$

(d) multiplication $\mu: \mathcal{S} \otimes_{\mathcal{O}} C_{\mathcal{A}}(\mathcal{S}) \rightarrow \mathcal{A}$ such that $\sigma \otimes_{\mathcal{O}} \rho \mapsto \sigma \rho=\rho \sigma$ for all $\sigma \in \mathcal{S}$

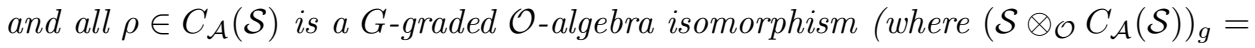
$\mathcal{S} \otimes_{\mathcal{O}}\left(C_{\mathcal{A}}(\mathcal{S})_{g}\right)$ for all $\left.g \in G\right)$; and

(e) $\mathcal{A} J\left(\mathcal{A}_{1}\right)$ is a $G$-graded ideal of $\mathcal{A}$ and, setting $\tilde{\mathcal{A}}=\mathcal{A} /\left(\mathcal{A} J\left(\mathcal{A}_{1}\right)\right)$, $\tilde{\mathcal{A}}$ becomes a $G$-graded crossed-product $k$-algebra with $\tilde{\mathcal{A}}_{g}=\mathcal{A}_{g}+\mathcal{A} J\left(\mathcal{A}_{1}\right)$ for all $g \in G$ and $\tilde{\mathcal{A}}_{1}=$ $\tilde{\mathcal{S}} \cong M_{r}(k)$ as $k$-algebras. Moreover, $C_{\tilde{\mathcal{A}}_{1}}\left(\tilde{\mathcal{A}}_{1}\right)=Z\left(\tilde{\mathcal{A}}_{1}\right)=\widetilde{\mathcal{O} 1_{\mathcal{A}}} \cong k, C_{\tilde{\mathcal{A}}}\left(\tilde{\mathcal{A}}_{1}\right)=$ $\oplus_{g \in G} C_{\tilde{\mathcal{A}}_{1}}\left(\tilde{\mathcal{A}}_{1}\right) \tilde{v}_{g}$ is a G-graded twisted group k-subalgebra of $\tilde{\mathcal{A}}=\oplus_{g \in G} \tilde{\mathcal{A}}_{g}$ and multiplication $\tilde{\mu}: \tilde{\mathcal{A}}_{1} \otimes_{k} C_{\tilde{\mathcal{A}}}\left(\tilde{\mathcal{A}}_{1}\right) \rightarrow \tilde{\mathcal{A}}$ such that $\tilde{\sigma} \otimes_{k} \tilde{\rho} \mapsto \tilde{\sigma} \tilde{\rho}=\tilde{\rho} \tilde{\sigma}$ for all $\tilde{\sigma} \in \tilde{\mathcal{A}}_{1}$ and all $\tilde{\rho} \in C_{\tilde{\mathcal{A}}}\left(\tilde{\mathcal{A}}_{1}\right)$ is a G-graded $k$-algebra isomorphism (where $\left(\tilde{\mathcal{A}}_{1} \otimes_{k} C_{\tilde{\mathcal{A}}}\left(\tilde{\mathcal{A}}_{1}\right)\right)_{g}=$ $\tilde{\mathcal{A}}_{1} \otimes_{k}\left(C_{\tilde{\mathcal{A}}}\left(\tilde{\tilde{\mathcal{A}}}_{1}\right)_{g}\right)$ for all $\left.g \in G\right)$.

Remark 1.16. In Proposition 1.15(e), $C_{\tilde{\mathcal{A}}}\left(\tilde{\mathcal{A}}_{1}\right)=\oplus_{g \in G} C_{\tilde{\mathcal{A}}_{1}}\left(\tilde{\mathcal{A}}_{1}\right) \tilde{v}_{g}$ is the "Clifford extension" of [3, (1.10) and (4.2)].

Proof. By [18, Theorem 7.3] and Proposition 1.12, we have (a). Fix $g \in G$. Since $u_{g} \mathcal{S}$ is also a maximal $\mathcal{O}$-semisimple subalgebra of $\mathcal{A}_{1}$, there is an element $j \in J\left(\mathcal{A}_{1}\right)$ such that $(1+j) u_{g} \mathcal{S}=\mathcal{S}$. $\operatorname{As} \operatorname{Aut}_{\mathcal{O}}(\mathcal{S})=\operatorname{Inn}_{\mathcal{O}}(\mathcal{S})$ (cf. [18. Theorem 7.2]), there is an element $s_{g} \in \mathcal{S}^{\times}$such that $s_{g}(1+j) u_{g} \in C_{\mathcal{A}_{g}}(\mathcal{S}) \cap \mathcal{A}^{\times}$and we may take $w_{g}=s_{g}(1+j) \in \mathcal{A}_{1}^{\times}$to conclude (b). Then (c) is immediate and (d) follows from [18, Proposition 7.5]. For similar reasons (e) also holds.

In the remainder of this section, $G$ will, as usual, denote a finite group and we shall also assume that $(\mathcal{K}, \mathcal{O}, k)$ is "big enough" for all subgroups of $G$. Let $b$ be a block idempotent of $\mathcal{O} G$ and let $-: \mathcal{O} G \rightarrow k G$ be the $\mathcal{O}$-algebra epimorphism 
induced by the natural epimorphism - : $\mathcal{O} \rightarrow k=\mathcal{O} / J(\mathcal{O})$. Thus $\bar{b}$ is a block idempotent of $k G$.

We shall need the following well-known "facts":

Lemma 1.17. (a) If $D$ is a defect group of b, then $D$ is a defect group of $\bar{b}$; and (b) if $P$ is a p-subgroup of $G$, then the $\mathcal{O} P$-module homorphism $m(b): \mathcal{O} P \rightarrow(\mathcal{O} G) b$ such that $p \mapsto p b$ for all $p \in P$ is an injection and the kP-module homorphism $\overline{m(b)}: k P \rightarrow(k G) \bar{b}$ induced by $m(b)$ is an injection.

Proof. Here (a) is a consequence of [5, III, Theorem 6.10]. Since $(\mathcal{O} G) b$ is projective and hence free in $\mathcal{O} P$-mod, the first part of (b) holds and similar arguments yield the remainder of (b).

Next assume that $b$ is a block of defect 0 of the finite group $G$ and let $V$ be an indecomposable module in $(\mathcal{O} G) b$-mod. Then $V$ is projective in $\mathcal{O} G b$-mod, $\bar{V}$ is irreducible in $k G b$-mod and $\mathcal{K} \otimes_{\mathcal{O}} V$ is irreducible in $\mathcal{K} G b$-mod. Let $\chi$ denote the character of $\mathcal{K} \otimes_{\mathcal{O}} V$ and let $\varphi$ denote the Brauer character of $\bar{V}$. Let $H \leq G$ and let $\beta$ be a block of defect 0 of $H$ and let $W$ be an indecomposable module in $(\mathcal{O} H) \beta$-mod so that we have similar conditions as above. Also let $\delta$ denote the character of $\mathcal{K} \otimes_{\mathcal{O}} W$ and let $\psi$ denote the Brauer character of $\bar{W}$. Finally let $\omega$ denote the multiplicity of $\bar{W}$ as a composition factor of $\operatorname{Res}_{H}^{G}(\bar{V})$.

Lemma 1.18. $\omega=\left(\operatorname{Res}_{H}^{G}(\chi), \delta\right)_{H}$.

Proof. Clearly $\omega=\frac{1}{|H|} \sum_{h \in H_{p^{\prime}}} \varphi(h) \psi\left(h^{-1}\right)$. Since $\chi$ and $\delta$ vanish on $p$-singular elements and $\chi$ and $\varphi$ and $\delta$ and $\psi$ agree on $p$-regular elements, we are done.

In the remainder of this section let the solvable finite group $\mathcal{A}$ act on $G$ on the right and be such that $(|G|,|A|)=1$ so that we are in the Glauberman correspondence situation (cf. [10, Chapter 13]). Set $C=C_{G}(A)$.

Let $N \unlhd G$ be $A$-invariant. Then $C_{N}(A)=C \cap N \unlhd C$ and $\pi(N, A): \operatorname{Irr}(N)^{A} \rightarrow$ $\operatorname{Irr}(C \cap N)$ is a bijection.

Lemma 1.19. Let $\chi \in \operatorname{Irr}(N)^{A}$ and let $c \in C$. Then:

(a) $\chi^{c} \in \operatorname{Irr}(N)^{A}$;

(b) $(\pi(N, A)(\chi))^{c}=\pi(N, A)\left(\chi^{c}\right)$; and

(c) $\operatorname{Stab}_{C}(\chi)=\operatorname{Stab}_{C}(\pi(N, A)(\chi))$.

Proof. Clearly (a) holds and, for (b), [10, Theorem 13.1] implies that we may assume that $A$ is a $q$-group for some prime $q \neq p$. Then $\pi(N, A)(\chi)$ is the unique irreducible constituent of $\operatorname{Res}_{C \cap N}^{N}(\chi)$ with multiplicity prime to $q$ by 10 , Theorem 13.4]. Thus $\pi(N, A)(\chi)^{c}$ is the unique irreducible constituent of $\operatorname{Res}_{C \cap N}^{N}\left(\chi^{c}\right)$ with multiplicity prime to $q$. Thus (b) follows and (c) is immediate.

Let $D$ be a $p$-subgroup of $C \cap N$ and let $\beta \in B \ell(N)^{A}$ have $D$ as a defect group. Then $W(\beta)$ is a block of $C \cap N$ with defect group $D$ and $\operatorname{Irr}(W(\beta))=\{\pi(N, A)(\chi) \mid$ $\chi \in \operatorname{Irr}(\beta)\}\left(\right.$ where $\left.\operatorname{Irr}(\beta) \subseteq \operatorname{Irr}(N)^{A}\right)$.

Our next result is an immediate consequence of Lemma 1.19.

Corollary 1.20. Let $c \in C$ (with $\beta$ and $D$ as above). Then:

(a) $\beta^{c} \in B \ell(N)^{A}$ and has $D^{c}$ as a defect group;

(b) $W\left(\beta^{c}\right)=W(\beta)^{c}$; and

(c) $\operatorname{Stab}_{C}(\beta)=\operatorname{Stab}_{C}(W(\beta))$.

We continue with the hypotheses above. 
Lemma 1.21. (a) Let $\beta \in B \ell(N)^{A}$ have $D$ as a defect group. Then there is an $A$-invariant block $b$ of $G$ that covers $\beta$ and such that $D=N \cap \Delta$ for some defect group $\Delta$ of b;

(b) assume that $D \unlhd N$ and let $b$ be an A-invariant block of $G$ with a defect group $D$. Then $D=O_{p}(G)=O_{p}(N)$ and there is an A-invariant block $\beta$ of $N$ covered by $b$ such that $D$ is a defect group of $\beta$; and

(c) let $b \in B \ell(G)^{A}$ have $D$ as a defect group and cover $\beta \in B \ell(N)^{A}$ such that $D$ is a defect group of $\beta$. Then $W(b) \in B \ell(C)$ (with defect group $D$ ) covers $W(\beta)$ of $C \cap N$ (with defect group $D$ ).

Proof. Assume the conditions of (a) and let $\psi \in \operatorname{Irr}(\beta)$, so that $\psi$ is $A$-stable by [19. Proposition 1]. Then [10, Theorem 13.28] yields an $A$-stable irreducible constituent $\chi$ of $\operatorname{Ind}_{N}^{G}(\psi)$ which must belong to an $A$-stable block $b$ of $G$ that covers $\beta$. Thus [12, Proposition 4.2], completes the proof.

Assume the conditions of (b). Thus $D \leq O_{p}(G)$ and hence $D=O_{p}(G)$ since $D$ is a defect group of $b$. Let $\chi \in \operatorname{Irr}(b)$ so that $\chi$ is $A$-stable. Here [10, Theorem 13.27] yields an $A$-stable irreducible constituent $\psi$ of $\operatorname{Res}_{N}^{G}(\chi)$. Then $\psi$ lies in an $A$-stable block $\beta$ of $N$. Let $\Delta$ be a defect group of $\beta$, so that $D=O_{p}(N) \leq \Delta$. As above $\Delta \leq \Delta^{\prime}$ for some defect group $\Delta^{\prime}$ of $b$. Since $|D|=\left|\Delta^{\prime}\right|$, we conclude that $D=\Delta=\Delta^{\prime}$ is a defect group of $\beta$.

Assume the conditions of (c), let $\chi \in \operatorname{Irr}(b)$ and let $\psi \in \operatorname{Irr}(\beta)$ be such that $\left[\operatorname{Res}_{N}^{G}(\chi), \psi\right]_{N} \neq 0$. Thus $\left[\chi, \operatorname{Ind}_{N}^{G}(\psi)\right]_{G} \neq 0$ and [10, Theorem 13.29] implies that $0 \neq\left[\operatorname{Ind}_{C \cap N}^{C}(\pi(N, A)(\psi)), \pi(G, A)(\chi)\right]_{C}=\left[\pi(N, A)(\psi), \operatorname{Res}_{C \cap N}^{C}(\pi(G, A)(\chi))\right]_{C \cap N}$. Thus $W(b)$ covers $W(\beta)$ and we are done.

For the final results of this section, we assume that $D \unlhd G$, where $D$ is a $p$ group. (These results may hold for arbitrary sets of primes.) Clearly if $g \in G$, then $D$ normalizes $g D=D g, G_{p^{\prime}}$ and $(g D) \cap G_{p^{\prime}}$.

Lemma 1.22. Let $g \in G$. Then the following five conditions are equivalent:

(a) $g D \in(G / D)_{p^{\prime}}$,

(b) $g_{p} \in D$,

(c) $x_{p} \in D$ for all $x \in g D$,

(d) $(g D) \cap G_{p^{\prime}} \neq \emptyset$; and

(e) $x D=x_{p^{\prime}} D$ for all $x \in g D$.

In which case, if $z \in(g D) \cap G_{p^{\prime}}$, then:

(f) $(g D) \cap G_{p^{\prime}}=z^{D}$;

(g) if $\mathcal{T}$ is a right transversal of $C_{D}(z)$ in $D$, then $g D=\bigcup_{t \in \mathcal{T}}\left(z C_{D}(z)\right)^{t}$, where the union is disjoint;

(h) if $\mathcal{T}$ is a right transversal of $G_{D}\left(g_{p^{\prime}}\right)$ in $D$, then $g D=\bigcup_{t \in \mathcal{T}}\left(g C_{D}\left(g_{p^{\prime}}\right)\right)^{t}$, where the union is disjoint; and

(i) if $\psi: g D \rightarrow \mathcal{K}$ is $D$-stable, then

$$
\sum_{x \in g D} \psi(x)=\left|D: C_{D}\left(g_{p^{\prime}}\right)\right|\left(\sum_{d \in C_{D}\left(g_{p^{\prime}}\right)} \psi(g d)\right) .
$$

Proof. The equivalence of (a)-(e) is well known and easy. Let $z \in(g D) \cap G_{p^{\prime}}$. Then $z^{D} \subseteq(g D) \cap G_{p^{\prime}}$ since $D$ normalizes $(g D) \cap G_{p^{\prime}}$. Let $u \in(g D) \cap G_{p^{\prime}}=(z D) \cap G_{p^{\prime}}$. Then $\langle u\rangle \leq D\langle z\rangle,\langle u\rangle$ is a $p^{\prime}$-subgroup and $\langle z\rangle$ is a $p^{\prime}$-subgroup complement to $D$ in $D\langle z\rangle$. Thus [7] Theorem 6.3.6] yields an element $d \in D$ such that $\langle u\rangle^{d} \leq\langle z\rangle$. 
Hence $u^{d} \in(z D) \cap\langle z\rangle=\{z\}$ and (f) holds. Let $u \in g D=z D$. Then $u=u_{p^{\prime}} u_{p}$, where $u_{p} \in D$ and $u_{p^{\prime}} \in(g D) \cap G_{p^{\prime}}=z^{D}$. Hence $u_{p^{\prime}}^{d}=z$ for some $d \in D$ and $u^{d}=z u_{p}^{d} \in z C_{D}(z)$. Thus $g D=\bigcup_{d \in D}\left(z C_{D}(z)\right)^{d}=\bigcup_{t \in \mathcal{T}}\left(z C_{D}(z)\right)^{t}$ since $C_{D}(z)$ normalizes $z C_{D}(z)$. Since $\left|\bigcup_{t \in \mathcal{T}}\left(z C_{D}(z)\right)^{t}\right| \leq\left|D: C_{D}(z)\right|\left|z C_{D}(z)\right|=|D|$, (g) holds. Note that $g=g_{p^{\prime}} g_{p}$, where $g_{p^{\prime}} \in(g D) \cap G_{p^{\prime}}$ and $g_{p} \in C_{D}\left(g_{p^{\prime}}\right)$. Then $g D=\bigcup_{t \in \mathcal{T}}\left(g_{p^{\prime}} C_{D}\left(g_{p^{\prime}}\right)\right)^{t}=\bigcup_{t \in \mathcal{T}}\left(g C_{D}\left(g_{p^{\prime}}\right)\right)^{t}$ and we are done.

Let $G_{p^{\prime}}=\bigcup_{i \in I} \mathcal{O}_{i}$ be the $D$-conjugation orbit decomposition of $G_{p^{\prime}}$.

Corollary 1.23. $\varphi:(G / D)_{p^{\prime}} \rightarrow\left\{\mathcal{O}_{i} \mid i \in I\right\}$ such that $x D \in(G / D)_{p^{\prime}} \mapsto(x D) \cap G_{p^{\prime}}$ is a bijection.

\section{Proofs of Theorems 1 And 2}

We begin with a proof of Theorem 1. Under the hypotheses of Theorem 1, let $H=N_{G}(D)$ and $K=D C_{G}(D) \unlhd H$. Then Lemma 1.21(b) yields a block $\beta \in B \ell(K)^{A}$ with defect group $D$ that is covered by $B r_{D}(b)$. Here $\beta$ is also a block of $C_{G}(D)$ and $(D, \beta)$ is a maximal $b$-subpair. By [19, Proposition $\left.4(\mathrm{i})\right],(D, W(\beta))$ is a maximal $W(b)$-subpair. Here $W(\beta)$ is a block of $D C_{C}(D)=C_{K}(A)$ and $W\left(B r_{D}(b)\right)$ covers $W(\beta) \in B \ell\left(C_{K}(A)\right)$ by Lemma $1.21(\mathrm{c})$. Since $(D, W(\beta))$ is a maximal $W(b)$-subpair, it follows that $B r_{D}(W(b))$ is the unique block of $N_{C}(D)$ that covers $W(\beta) \in B \ell\left(C_{K}(A)\right)$. Thus $W\left(B r_{D}(b)\right)=B r_{D}(W(b))$ and we are done.

Next we proceed to demonstrate Theorem 2. So we assume that $D \unlhd G$. Let $A, G, D \unlhd C=C_{G}(A), b \in B \ell(G)^{A}$ and $W(b) \in B \ell(C)$ be as in Theorem 2. Set $L=C_{G}(D) \unlhd G$ and $K=D C_{G}(D) \unlhd G$ and note that $D=O_{p}(G)$. By [10] Theorem 13.27], there is an $A$-stable block $\beta$ of $K$ that is covered by $b$. Since $D=O_{p}(K)$, 12, Proposition 4.2] implies that $D$ is the defect group of $\beta$. Let $T=\operatorname{Stab}_{G}(\beta)$ so that $K=D C_{G}(D) \leq T \leq G$ and $T$ is $A$-invariant. Set $L_{1}=$ $L \cap C=C_{C}(D) \unlhd C$ and $K_{1}=K \cap C=D C_{C}(D) \unlhd C$, so that $W(\beta) \in B \ell\left(K_{1}\right)$ has defect group $D$. Also $T \cap C=\operatorname{Stab}_{C}(\beta)=\operatorname{Stab}_{C}(W(\beta))$ by Corollary 1.20(c). As is well known, there is a unique block $b_{T}$ of $T$ that covers $\beta$ and is such that $\left\{\operatorname{Ind}_{T}^{G}(\psi) \mid \psi \in \operatorname{Irr}\left(b_{T}\right)\right\}=\operatorname{Irr}(b)$ and $b_{T}$ induces $b$ via Brauer block induction. Also $b_{T}$ is $A$-stable and has defect group $D$. Moreover, $(\mathcal{O} G) b \cong \operatorname{Ind}_{T}^{G}\left((\mathcal{O} T) b_{T}\right)$ as interior $G$-algebra and as $A$-algebras, $b=\operatorname{Tr}_{T}^{G}\left(b_{T}\right)$, and the Brauer categories of $(\mathcal{O} G) b$ and $(\mathcal{O} T) b_{T}$ are equivalent, and $(\mathcal{O} T) b_{T}$ and $(\mathcal{O} G) b$ are Morita equivalent $\mathcal{O}$ algebras by a categeorical equivalence that on the character level yields the bijection $\operatorname{Ind}_{T}^{G}: \operatorname{Irr}\left(b_{T}\right) \rightarrow \operatorname{Irr}(b)$.

Here $W(b) \in B \ell(C)$ covers $W(\beta)$ and both have defect group $D, W\left(b_{T}\right) \in$ $B \ell(T \cap C), W\left(b_{T}\right)$ covers $W(\beta)$ and also has defect group $D$ by [10, Theorem 13.29]. Moreover, $\operatorname{Irr}(W(b))=\left\{W\left(\operatorname{Ind}_{T}^{G}(\psi)\right) \mid \psi \in \operatorname{Irr}\left(b_{T}\right)\right\}=\left\{\operatorname{Ind}_{T \cap C}^{C}(\pi(T, A)(\psi)) \mid \psi \in\right.$ $\left.\operatorname{Irr}\left(b_{T}\right)\right\}$ by [8, Theorem 2.3(ii)]. Thus $(\mathcal{O} C) W(b) \cong \operatorname{Ind}_{C \cap T}^{C}\left((\mathcal{O}(C \cap T)) W\left(b_{T}\right)\right)$ as interior $C$-algebras, $W(b)=\operatorname{Tr}_{C \cap T}^{C}\left(W\left(b_{T}\right)\right)$, and the Brauer categories of $(\mathcal{O} C) W(b)$ and $(\mathcal{O}(C \cap T)) W\left(b_{T}\right)$ are equivalent, and $W\left(b_{T}\right)$ induces $W(b)$ via Brauer block induction, and $\mathcal{O}(C \cap T) W\left(b_{T}\right)$ and $(\mathcal{O} C) W(b)$ are Morita equivalent $\mathcal{O}$-algebras via a categorical equivalence that on the character level yields the bijection $\operatorname{Ind}_{C \cap T}^{C}$ : $\operatorname{Irr}\left(W\left(b_{T}\right)\right) \rightarrow \operatorname{Irr}(W(b))$.

Consequently it suffices to assume that $\beta$ is $G$-stable. In that case, $\beta=b$ and $b$ is a block idempotent of $\mathcal{O} G, \mathcal{O} K$ and $\mathcal{O} L$, where $Z(D)$ is a defect group of $b$ as a block of $\mathcal{O} L$. Also $|G / K|=m$ is relatively prime to $p$. Similarly $W(\beta)=W(b)$ 
is a block idempotent of $\mathcal{O} C, \mathcal{O} K_{1}$ and $\mathcal{O} L_{1}$ where $Z(D)$ is a defect group of $W(b)$ as a block of $\mathcal{O} L_{1}$ (cf. [5, V, Sections 3 and 4]).

Since $[G, A] \leq C_{G}(D)$, we have $G=C_{G}(D) C$. Since $D C_{C}(D) / C_{C}(D)$ is a normal Sylow $p$-subgroup of $C / C_{C}(D)$, there is a subgroup $H_{1}$ of $C$ with $C_{C}(D) \leq$ $H_{1}, C=H_{1} D$ and $H_{1} \cap D=Z(D)$. Thus the map $h C_{C}(D) \mapsto h K$ for $h \in H_{1}$ is an isomorphism of $H_{1} / L_{1}$ onto $G / K$. Let $\mathcal{T}$ be a transversal of $L_{1}$ in $H_{1}$ with $1 \in \mathcal{T}$, so that $\mathcal{T}$ is a transversal of $K$ in $G$ and $|\mathcal{T}|=\left|H_{1} / L_{1}\right|=|G / K|=\left|C / K_{1}\right|=m$.

Set $H=C_{G}(D) H_{1}$, so that $L \leq H \leq G=H D$ and $H \cap D=Z(D), H$ is $A$-invariant, $C_{H}(A)=C \cap H=H_{1}$ and $H_{1} \cap C_{G}(D)=C_{C}(D)$. As $L_{1}=C_{H_{1}}(D)$, $H_{1} / L_{1}$ acts faithfully by conjugation on $D$. Let $\mathcal{N}=D \rtimes\left(H_{1} / L_{1}\right)$.

As is well known, the block $b$ of $\mathcal{O} K$ contains exactly one irreducible character $\theta$ such that $D \leq \operatorname{Ker}(\theta)$ and $b$ contains exactly one irreducible Brauer character $\varphi$ and $\varphi(x)=\theta(x)$ for all $p^{\prime}$-elements $x$ of $K$. Let $V$ be an $\mathcal{O}$-free $\mathcal{O} K$-module that affords $\theta$ and let $r=\theta(1)=\operatorname{rank}(V / \mathcal{O})$. Then $D \leq \operatorname{Ker}(V)$ and $\bar{V}$ is an irreducible $k K$-module in $b$ with Brauer character $\varphi$. Let $P(\bar{V})$ denote a projective indecomposable $(\mathcal{O} K) b$-module corresponding to $\bar{V}$ and let $\Phi$ be the character of $P(\bar{V})$. Then $\Phi(x)=|D| \varphi(x)=|D| \theta(x)$ for all $p^{\prime}$-elements $x$ of $K$ by [5] V, Corollary 4.6] and hence $\operatorname{rank}(P(\bar{V}) / \mathcal{O})=r|D|$. Since $(\mathcal{O} K) b \cong P(\bar{V})^{r}$ in $(\mathcal{O} K) b$-mod, we conclude that $\operatorname{rank}((\mathcal{O} K) b / \mathcal{O})=r^{2}|D|$. Note that $\operatorname{Res}_{L}^{K}(\theta)$ is the unique irreducible character in the block $b$ of $L$ with $Z(D) \leq \operatorname{Ker}\left(\operatorname{Res}_{L}^{K}(\theta)\right)$; we similarly conclude that $\operatorname{rank}((\mathcal{O} L) b / \mathcal{O})=r^{2}|Z(D)|$. Also $\bar{\theta}=\operatorname{char}(\bar{V})$ is the unique irreducible character of $(k K) \bar{b}$, and $\operatorname{Res}_{L}^{K}(\bar{\theta})=\operatorname{char}\left(\operatorname{Res}_{L}^{K}(\bar{V})\right)$ is the unique irreducible character of $(k L) \bar{b}$ and $r_{p}=|K / D|_{p}=|G / D|_{p}$.

Clearly $(\mathcal{O} L) b,(\mathcal{O} K) b$ and $(\mathcal{O} H) b$ are $\mathcal{O}$-subalgebras of $(\mathcal{O} G) b$.

Let $\theta_{1}, \varphi_{1}, V_{1}, r_{1}=\theta_{1}(1), \bar{V}_{1}, P\left(\bar{V}_{1}\right), \Phi_{1}$ be the corresponding objects of the block $W(b)$ of $K_{1}$ with defect group $D$. Note that $\pi(K, A)(\theta)=\theta_{1}$ since $D \leq$ $\operatorname{ker}(\pi(K, A)(\theta))$ and $\pi(K, A)(\theta) \in \operatorname{Irr}_{k}(W(b))$.

Proposition 2.1. (a) the $\mathcal{O}$-algebra homomorphism $M(b): \mathcal{O} D \rightarrow(\mathcal{O} K) b$ such that $\alpha \mapsto \alpha b$ for all $\alpha \in \mathcal{O} D$ is a $G$-injection such that $M(b)(\mathcal{O} Z(D))=(\mathcal{O} Z(D)) b \leq$ $(\mathcal{O} L) b$

(b) there is an $\mathcal{O}$-simple subalgebra $\mathcal{S}$ of $(\mathcal{O} L)$ b such that $b \in \mathcal{S}, \mathcal{S} \cong M_{r}(\mathcal{O})$ as $\mathcal{O}$-algebras, $(\mathcal{O} L) b=\mathcal{S}+J((\mathcal{O} L) b),(\mathcal{O} K) b=\mathcal{S}+J((\mathcal{O} K) b), \mathcal{S}$ is a maximal $\mathcal{O}$ semi-simple $\mathcal{O}$-subalgebra of both $(\mathcal{O} L) b$ and $(\mathcal{O} K) b$, and $J(\mathcal{O}) \mathcal{S}=\mathcal{S} \cap J((\mathcal{O} K) b)=$ $\mathcal{S} \cap J((\mathcal{O} L) b) ;$

(c) $C_{(\mathcal{O} K) b}(\mathcal{S})=(\mathcal{O} D) b$ and $C_{(\mathcal{O} L) b}(\mathcal{S})=(\mathcal{O} Z(D)) b$ and the $\mathcal{O}$-linear "multiplication maps" $\mu: \mathcal{S} \otimes_{\mathcal{O}}((\mathcal{O} D) b) \rightarrow(\mathcal{O} K) b$ such that $s \otimes_{\mathcal{O}} \alpha \mapsto s \alpha$ for all $s \in \mathcal{S}$ and all $\alpha \in(\mathcal{O} D) b$, and $\mu: \mathcal{S} \otimes(\mathcal{O} Z(D)) b \rightarrow(\mathcal{O} L) b$ such that $s \otimes_{\mathcal{O}} \alpha \mapsto s \alpha$ for all $s \in \mathcal{S}$ and all $\alpha \in(\mathcal{O} Z(D)) b$ are $\mathcal{O}$-algebra isomorphisms;

(d) $(\mathcal{O} G) b=\oplus_{t \in \mathcal{T}}((\mathcal{O} K) b)(t b)$ and $(\mathcal{O} H) b=\oplus_{t \in \mathcal{T}}((\mathcal{O} L) b)(t b)$ exhibit $(\mathcal{O} G) b$ and $(\mathcal{O} H) b$ as $H_{1} / L_{1}$-crossed product $\mathcal{O}$-algebras with $((\mathcal{O} G) b)_{t L_{1}}=((\mathcal{O} K) b)(t b)$ and $((\mathcal{O} H) b)_{t L_{1}}=((\mathcal{O} L) b)(t b)$ for all $t \in \mathcal{T}$, respectively;

(e) for each $t \in \mathcal{T}$, there is an element $w_{t} \in((\mathcal{O} L) b)^{\times}$such that

$$
\begin{gathered}
v_{t}=w_{t}(t b) \in C_{((\mathcal{O} L) b)(t b)}(\mathcal{S}) \cap((\mathcal{O} H) b)^{\times}, v_{1}=w_{1}=b, \\
C_{(O H) b}(\mathcal{S})=\oplus_{t \in \mathcal{T}}\left(((\mathcal{O} Z(D)) b) v_{t}\right), \\
C_{(\mathcal{O} G) b}(\mathcal{S})=\oplus_{t \in \mathcal{T}}\left(((\mathcal{O} D) b) v_{t}\right)
\end{gathered}
$$

and $v_{t} \alpha v_{t}^{-1}=t \alpha t^{-1}={ }^{t} \alpha$ for all $\alpha \in(\mathcal{O} D) b, v_{t} \alpha v_{t}^{-1}=w_{t}\left({ }^{t} \alpha\right) w_{t}^{-1}$ for all $\alpha \in$

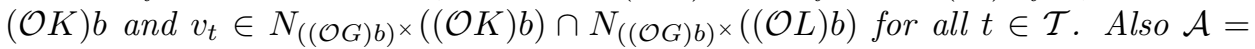


$\oplus_{t \in \mathcal{T}} \mathcal{O} v_{t}$ is a twisted $H_{1} / L_{1}$-group $\mathcal{O}$-subalgebra of $(\mathcal{O} H) b$ with associated $c \in$ $Z^{2}\left(H_{1} / L_{1}, f\left(k^{\times}\right)\right)$such that $c^{m}=1$ and $c\left(t L_{1}, L_{1}\right)=c\left(L_{1}, t L_{1}\right)=1$ for all $t \in \mathcal{T}$, $\Omega=\left\{c\left(t_{1} L_{1}, t_{2} L_{1}\right) \mid t_{1}, t_{2} \in \mathcal{T}\right\}$ is a finite subgroup of $\mathcal{O}^{\times}$of order $n$ dividing $m$ and $\left\{\omega v_{t} \mid \omega \in \Omega, t \in \mathcal{T}\right\}$ is a subgroup of $\mathcal{A}^{\times}$of order $m n$;

(f) multiplication $\mu: \mathcal{S} \otimes_{\mathcal{O}} C_{(\mathcal{O} G) b}(\mathcal{S}) \rightarrow(\mathcal{O} G) b$ such that $s \otimes \alpha \mapsto s \alpha$ for all $s \in \mathcal{S}$ and all $\alpha \in C_{(\mathcal{O} G) b}(\mathcal{S})$ is an $\mathcal{O}$-algebra isomorphism;

(g) $(\mathcal{O} H) b J((\mathcal{O} L) b)$ is an $H_{1} / L_{1}$-graded ideal of $(\mathcal{O H}) b$ and hence $\widetilde{(\mathcal{O} H) b}=$ $(\mathcal{O H}) b /((\mathcal{O H}) b J((\mathcal{O} L) b))$ is an $H_{1} / L_{1}$-crossed product $k$-algebra with $(\widetilde{(\widetilde{\mathcal{O} H}) b})_{t L_{1}}=$ $[((\mathcal{O} L) b)(t b)+(\mathcal{O} H) b J((\mathcal{O} L) b)] /[(\mathcal{O H}) b J((\mathcal{O} L) b)]$ for all $t \in \mathcal{T}$ and $(\widetilde{(\mathcal{O} H)} b)_{L_{1}} \cong$ $\mathcal{S} / J(\mathcal{O}) \mathcal{S} \cong M_{r}(k)$ as k-algebras. Moreover, the injection $i: \mathcal{A}=\oplus_{t \in \mathcal{T}} \mathcal{O} v_{t} \rightarrow$ $(\mathcal{O} H) b$ induces an $H_{1} / L_{1}$-graded isomorphism of $\overline{\mathcal{A}}=\mathcal{A} / J(\mathcal{O}) \mathcal{A}$ onto the associated "Clifford extension" of $(\mathcal{O H}) b$ with respect to $(\mathcal{O} H) b J((\mathcal{O} L) b)$; and

(h) let $\mathcal{L}$ denote the $\mathcal{O}$-free twisted $\mathcal{N}$-group algebra with $\mathcal{O}$-free basis $\left\{\left(d, t L_{1}\right) \mid\right.$ $d \in D, t \in \mathcal{T}\}$ such that $\left(d_{1}, t_{1} L_{1}\right)\left(d_{2}, t_{2} L_{1}\right)=c\left(t_{1} L_{1}, t_{2} L_{1}\right)\left(d_{1}\left({ }^{t_{1}} d_{2}\right), t_{3} L_{1}\right)$, where $d_{1}, d_{2} \in D$ and $t_{1}, t_{2} \in \mathcal{T}$ and $\left(t_{1} L_{1}\right)\left(t_{2} L_{1}\right)=t_{3} L_{1}$ for a unique $t_{3} \in \mathcal{T}$. Then $\mathcal{L}$ is $\mathcal{O}$-algebra isomorphic to $C_{(\mathcal{O} G) b}(\mathcal{S})=\oplus_{t \in \mathcal{T}}\left(((\mathcal{O} D) b) v_{t}\right)$ via the $\mathcal{O}$-linear map such that $\left(d, t L_{1}\right) \mapsto(d b) v_{t}$ for all $d \in D$ and all $t \in \mathcal{T}$.

Remark 2.2. The "Clifford extension" of Proposition 2.1(g) is the "Clifford extension" of $k H$ with respect to the irreducible character $\operatorname{Res}_{L}^{K}(\bar{\theta})=\operatorname{char}\left(\operatorname{Res}_{L}^{K}(\bar{V})\right)$ of $k L$.

Proof. Clearly Lemma 1.17 yields (a). Since $\varphi$ is the unique irreducible Brauer character of $b \in B \ell(\mathcal{O} K)$ and $\operatorname{Res}_{L}^{K}(\varphi)$ is the unique irreducible Brauer character of $b \in B \ell(\mathcal{O} L)$, Propositions 1.12 and 1.15 and [18, Theorem 7.3] yield (b). Here the $\mathcal{O}$-linear "multiplication" map $\mu: \mathcal{S} \otimes C_{(\mathcal{O} K) b}(\mathcal{S}) \rightarrow(\mathcal{O} K) b$ is an $\mathcal{O}$-algebra isomorphism by [18, Proposition 7.5]. Thus $\operatorname{rank}\left(C_{(\mathcal{O} K) b}(\mathcal{S}) / \mathcal{O}\right)=$ $|D|=\operatorname{rank}((\mathcal{O} D) b / \mathcal{O})$ and $(\mathcal{O} D) b \leq C_{(\mathcal{O} K) b}(\mathcal{S})$. Also "reducing $\bmod p$ ", we similarly have $\operatorname{dim}((k D) \bar{b} / k)=|D|=\operatorname{dim}\left(C_{k K \bar{b}}(\overline{\mathcal{S}}) / k\right)$. Thus $C_{k K \bar{b}}(\overline{\mathcal{S}})=(k D) \bar{b}$ and $(\mathcal{O} D) b \leq C_{(\mathcal{O} K) b}(\mathcal{S}) \leq(\mathcal{O} D) b+J(\mathcal{O})(\mathcal{O} K) b$. Let $0 \neq x \in J(\mathcal{O})(\mathcal{O} K) b \cap C_{(\mathcal{O} K) b}(\mathcal{S})$. Then there are elements $0 \neq j \in J(\mathcal{O})$ and $0 \neq u \in(\mathcal{O} K) b$ such that $x=j u$ and we readily conclude that $u \in C_{(\mathcal{O} K) b}(\mathcal{S})$. Thus $(\mathcal{O} D) b \leq C_{(\mathcal{O} K) b}(\mathcal{S}) \leq$ $(\mathcal{O} D) b+J(\mathcal{O}) C_{(\mathcal{O} K) b}(\mathcal{S})$ and Nakayama's Lemma implies that $C_{(\mathcal{O} K) b}(\mathcal{S})=(\mathcal{O} D) b$. Similarly $C_{(\mathcal{O} L) b}(\mathcal{S})=(\mathcal{O} Z(D)) b$ and (c) holds. Clearly (d) holds.

For (e), recall that $1 \in \mathcal{T}$ and that $((\mathcal{O} L) b)^{\times}=\mathcal{S}^{\times}(b+J((\mathcal{O} L) b))=$ $(b+J(\mathcal{O} L) b)) \mathcal{S}^{\times}$by Proposition 1.12.

For each $t \in \mathcal{T}$, there is an element $w_{t}^{\prime} \in((\mathcal{O} L) b)^{\times}$such that

$$
w_{t}^{\prime}(t b) \in C_{(\mathcal{O} L) b(t b)}(\mathcal{S}) \cap((\mathcal{O} H) b)^{\times},
$$

where we may assume that $w_{1}^{\prime}=b$ if $t=1$ by Proposition 1.15(b). Consequently $(\mathcal{O} H) b=\oplus_{t \in \mathcal{T}}(\mathcal{O} L) b\left(\left(w_{t}^{\prime}(t b)\right)\right.$ and $C_{(\mathcal{O} H) b}(\mathcal{S})=\oplus_{t \in \mathcal{T}} \mathcal{O} Z(D) b\left(w_{t}^{\prime}(t b)\right)$, where $\mathcal{O} Z(D) b$ is a commutative $\mathcal{O}$-algebra. Lemma 1.13 implies that there is a set $\left\{u_{t} \mid t \in \mathcal{T}\right\} \subseteq(\mathcal{O} Z(D) b)^{\times}$such that $\oplus_{t \in \mathcal{T}} \mathcal{O}\left(u_{t} w_{t}^{\prime}(t b)\right)$ is a twisted $H_{1} / L_{1}$-group $\mathcal{O}$-algebra with associated $c \in Z^{2}\left(H_{1} / L_{1}, f\left(k^{\times}\right)\right)$. Then, since $k^{\times}$is $m=\left|H_{1} / L_{1}\right|$ divisible, Lemma 1.4 implies that we may replace each $u_{t}$ by an element of $\mathcal{O}^{\times} u_{t}$ to assure that $c^{m}=1$. Then, referring to Lemma 1.2, we may also assume that $c\left(t L_{1}, L_{1}\right)=c\left(L_{1}, t L_{1}\right)=1$ for all $t \in \mathcal{T}$. Then $\left(u_{1} b\right)\left(u_{1} b\right)=\left(u_{1} b\right)$ and hence $u_{1}=b$ and with $w_{t}=u_{t} w_{t}^{\prime}$ for all $t \in \mathcal{T}$ we have (e).

Finally [18, Proposition 7.5] implies (f), and (g) and (h) are clear. 
For similar reasons, we have:

Proposition 2.3. (a) the $\mathcal{O}$-algebra homomorphism $M(W(b)): \mathcal{O} D \rightarrow\left(\mathcal{O} K_{1}\right) W(b)$ such that $\alpha \mapsto \alpha W(b)$ for all $\alpha \in \mathcal{O} D$ is a $C$-injection such that $M(W(b))(\mathcal{O} Z(D))$ $=(\mathcal{O} Z(D)) W(b) \leq\left(\mathcal{O} L_{1}\right) W(b)$,

(b) there is an $\mathcal{O}$-simple subalgebra $\mathcal{S}_{1}$ of $\left(\mathcal{O} L_{1}\right) W(b)$ such that $W(b) \in \mathcal{S}_{1}, \mathcal{S}_{1} \cong$ $M_{r_{1}}(\mathcal{O})$ as $\mathcal{O}$-algebras, $\left(\mathcal{O} L_{1}\right) W(b)=\mathcal{S}_{1}+J\left(\left(\mathcal{O} L_{1}\right) W(b)\right), \quad\left(\mathcal{O} K_{1}\right) W(b)=\mathcal{S}_{1}+$ $J\left(\left(\mathcal{O} K_{1}\right) W(b)\right), \mathcal{S}_{1}$ is a maximal $\mathcal{O}$-semi-simple $\mathcal{O}$-subalgebra of both $\left(\mathcal{O} L_{1}\right) W(b)$ and $\left(\mathcal{O} K_{1}\right) W(b)$ and $J(\mathcal{O}) \mathcal{S}_{1}=\mathcal{S}_{1} \cap J\left(\left(\mathcal{O} K_{1}\right) W(b)\right)=\mathcal{S}_{1} \cap J\left(\left(\mathcal{O} L_{1}\right) W(b)\right)$;

(c) $C_{\left(\mathcal{O} K_{1}\right) W(b)}\left(\mathcal{S}_{1}\right)=(\mathcal{O} D) W(b)$ and $C_{\left(\mathcal{O} L_{1}\right) W(b)}\left(\mathcal{S}_{1}\right)=(\mathcal{O Z}(D)) W(b)$ and the $\mathcal{O}$-linear "multiplication maps" $\mu: \mathcal{S}_{1} \otimes_{\mathcal{O}}((\mathcal{O} D) W(b)) \rightarrow\left(\mathcal{O} K_{1}\right) W(b)$ such that $s_{1} \otimes_{\mathcal{O}} \alpha \mapsto s_{1} \alpha$ for all $s_{1} \in \mathcal{S}_{1}$ and all $\alpha \in(\mathcal{O} D) W(b)$, and $\mu: \mathcal{S}_{1} \otimes_{\mathcal{O}}$ $((\mathcal{O} Z(D)) W(b)) \rightarrow\left(\mathcal{O} L_{1}\right) W(b)$ such that $s_{1} \otimes_{\mathcal{O}} \alpha \mapsto s_{1} \alpha$ for all $s_{1} \in \mathcal{S}_{1}$ and all $\alpha \in(\mathcal{O} Z(D)) W(b)$ are $\mathcal{O}$-algebra isomorphisms;

(d)

$$
(\mathcal{O C}) W(b)=\oplus_{t \in \mathcal{T}}\left(\left(\mathcal{O} K_{1}\right) W(b)\right)(t W(b))
$$

and

$$
\left(\mathcal{O} H_{1}\right) W(b)=\oplus_{t \in \mathcal{T}}\left(\left(\mathcal{O} L_{1}\right) W(b)\right)(t W(b))
$$

exhibit $(\mathcal{O} C) W(b)$ and $\left(\mathcal{O} H_{1}\right) W(b)$ as $H_{1} / L_{1}$-crossed product $\mathcal{O}$-algebras with

$$
((\mathcal{O} C) W(b))_{t L_{1}}=\left(\left(\mathcal{O} K_{1}\right) W(b)\right)(t W(b))
$$

and

$$
\left(\left(\mathcal{O} H_{1}\right) W(b)\right)_{t L_{1}}=\left(\left(\mathcal{O} L_{1}\right) W(b)\right)(t W(b))
$$

for all $t \in \mathcal{T}$;

(e) for each $t \in \mathcal{T}$, there is an element $w_{t}^{\prime} \in\left(\left(\mathcal{O} L_{1}\right) W(b)\right)^{\times}$such that

$$
\begin{gathered}
v_{t}^{\prime}=w_{t}^{\prime}(t W(b)) \in C_{\left(\mathcal{O} L_{1}\right) W(b)(t W(b))}\left(\mathcal{S}_{1}\right) \cap\left(\left(\mathcal{O} H_{1}\right) W(b)\right)^{\times}, \quad v_{1}^{\prime}=w_{1}^{\prime}=W(b), \\
C_{\left(\mathcal{O} H_{1}\right) W(b)}\left(\mathcal{S}_{1}\right)=\oplus_{t \in \mathcal{T}}\left(((\mathcal{O} Z(D)) W(b)) v_{t}^{\prime}\right), \\
C_{(\mathcal{O} C) W(b)}\left(\mathcal{S}_{1}\right)=\oplus_{t \in \mathcal{T}}\left(((\mathcal{O} D) W(b)) v_{t}^{\prime}\right),
\end{gathered}
$$

$v_{t}^{\prime} \alpha\left(v_{t}^{\prime}\right)^{-1}=t \alpha t^{-1}={ }^{t} \alpha$ for all $\alpha \in(\mathcal{O} D) W(b), v_{t}^{\prime} \alpha\left(v_{t}^{\prime}\right)^{-1}=\left(w_{t}^{\prime}\right)\left({ }^{t} \alpha\right)\left(w_{t}^{\prime}\right)^{-1}$ for all

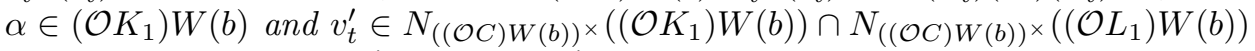
for all $t \in \mathcal{T}$. Also $\mathcal{A}^{\prime}=\oplus_{t \in \mathcal{T}} \mathcal{O} v_{t}^{\prime}$ is a twisted $H_{1} / L_{1}$-group $\mathcal{O}$-subalgebra of $\left(\mathcal{O} H_{1}\right) W(b)$ with associated $c^{\prime} \in Z^{2}\left(H_{1} / L_{1}, f\left(k^{\times}\right)\right)$such that $\left(c^{\prime}\right)^{m}=1$ and $c^{\prime}\left(t L_{1}, L_{1}\right)=c^{\prime}\left(L_{1}, t L_{1}\right)=1$ for all $t \in \mathcal{T}$;

(f) multiplication

$$
\mu_{1}: \mathcal{S}_{1} \otimes C_{(\mathcal{O} C) W(b)}\left(\mathcal{S}_{1}\right) \rightarrow(\mathcal{O} C) W(b)
$$

such that $s_{1} \otimes \alpha \mapsto s_{1} \alpha$ for all $s_{1} \in \mathcal{S}_{1}$ and all $\alpha \in C_{(\mathcal{O C}) W(b)}\left(\mathcal{S}_{1}\right)$ is an $\mathcal{O}$-algebra isomorphism;

(g) $\left(\mathcal{O} H_{1}\right) W(b) J\left(\left(\mathcal{O} L_{1}\right) W(b)\right)$ is an $H_{1} / L_{1}$-graded ideal of $\left(\mathcal{O} H_{1}\right) W(b)$ and hence $\left(\mathcal{O} \widetilde{\left.H_{1}\right) W}(b)=\left(\left(\mathcal{O} H_{1}\right) W(b)\right) /\left(\left(\mathcal{O} H_{1}\right) W(b) J\left(\left(\mathcal{O} H_{1}\right) W(b)\right)\right)\right.$ is an $H_{1} / L_{1}$-crossed product k-algebra with

$$
\begin{gathered}
\left(\left(\widetilde{\left.\mathcal{O} H_{1}\right)} W(b)\right)_{t L_{1}}=\left[\left(\left(\mathcal{O} L_{1}\right) W(b)(t b)+\left(\mathcal{O} H_{1}\right) W(b) J\left(\left(\mathcal{O} L_{1}\right) W(b)\right)\right]\right.\right. \\
/\left[\left(\mathcal{O} H_{1}\right) W(b) J\left(\left(\mathcal{O} L_{1}\right) W(b)\right)\right]
\end{gathered}
$$


for all $t \in \mathcal{T}$ and $\left(\widetilde{\mathcal{O}} \widetilde{\left.H_{1}\right) W}(b)_{L_{1}} \cong \mathcal{S}_{1} /\left(J(\mathcal{O}) \mathcal{S}_{1}\right) \cong M_{r_{1}}(k)\right.$ as $k$-algebras. Moreover, the injection $i^{\prime}: \mathcal{A}^{\prime}=\oplus_{t \in \mathcal{T}} \mathcal{O} v_{t}^{\prime} \rightarrow\left(\mathcal{O} H_{1}\right) W(b)$ induces an $H_{1} / L_{1}$-graded isomorphism of $\mathcal{A}^{\prime}=\mathcal{A}^{\prime} /\left(J(\mathcal{O}) \mathcal{A}^{\prime}\right)$ onto the associated "Clifford extension" of $\left(\mathcal{O} H_{1}\right) W(b)$ with respect to $\left(\mathcal{O} H_{1}\right) W(b) J\left(\left(\mathcal{O} L_{1}\right) W(b)\right)$; and

(h) let $\mathcal{L}^{\prime}$ denote the $\mathcal{O}$-free twisted $\mathcal{N}$-group algebra with $\mathcal{O}$-free basis $\left\{\left(d, t L_{1}\right) \mid\right.$ $d \in D, t \in \mathcal{T}\}$ such that $\left(d_{1}, t_{1} L_{1}\right)\left(d_{2}, t_{2} L_{1}\right)=c^{\prime}\left(t_{1} L_{1}, t_{2} L_{1}\right)\left(d_{1}\left({ }^{t_{1}} d_{2}\right), t_{3} L_{1}\right)$, where $d_{1}, d_{2} \in D$ and $t_{1}, t_{2} \in \mathcal{T}$ and $\left(t_{1} L_{1}\right)\left(t_{2} L_{1}\right)=t_{3} L_{1}$ for a unique $t_{3} \in \mathcal{T}$. Then $\mathcal{L}^{\prime}$ is $\mathcal{O}$-algebra isomorphic to $C_{(\mathcal{O C}) W(b)}\left(\mathcal{S}_{1}\right)=\oplus_{t \in \mathcal{T}}((\mathcal{O} D) W(b)) v_{t}^{\prime}$ via the $\mathcal{O}$-linear map such that $\left(d, t L_{1}\right) \mapsto d W(b) v_{t}^{\prime}$ for all $d \in D$ and all $t \in \mathcal{T}$.

Remark 2.4. The "Clifford extension" of Proposition 2.3(g) is the "Clifford extension" of $k H_{1}$ with respect to the irreducible character $\operatorname{Res}_{L_{1}}^{K_{1}}\left(\bar{\theta}_{1}\right)=\operatorname{char}\left(\operatorname{Res}_{L_{1}}^{K_{1}}\left(\bar{V}_{1}\right)\right)$ of $k L_{1}$.

By Propositions 2.1 and 2.3, a Morita equivalence will follow from the proof that the $\mathcal{O}$-free twisted $H_{1} / L_{1}$-group algebras $\mathcal{A}$ and $\mathcal{A}^{\prime}$ are $H_{1} / L_{1}$-graded isomorphic $\mathcal{O}$-algebras. Applying Propositions 2.1 and 2.3, Remarks 2.2 and 2.4 and Lemma 1.13(b), it suffices to prove that the associated "Clifford extensions" of $(\mathcal{O H}) b$ with respect to $(\mathcal{O} H) b J((\mathcal{O} L) b)$ and of $\left(\mathcal{O} H_{1}\right) W(b)$ with respect to $\left(\mathcal{O} H_{1}\right) W(b) J\left(\left(\mathcal{O} L_{1}\right) W(b)\right)$ are isomorphic.

At this point, we observe that it suffices to assume that $A$ is cyclic of prime order $q$.

Consequently, we can apply the proof of [14 Proposition 3.3] that is a consequence of [14, Lemma 3.2] of E.C. Dade working over $k$ as follows.

Let $\tilde{H}=H \rtimes A$ and $\tilde{L}=L \rtimes A$, so that $L \unlhd \tilde{H}$ and $\tilde{L} \unlhd \tilde{H}$ since $H=L H_{1}$ and $A$ centralizes $H_{1}$. We note here that $(\mathcal{K}, \mathcal{O}, k)$ is "big enough" for all subgroups of $\tilde{H}$. As $\gamma=\operatorname{Res}_{L}^{K}(\theta) \in \operatorname{Irr}(L)^{\tilde{H}}$ and $(|L|,|A|)=1, \gamma$ has a unique canonical extension $\tilde{\gamma} \in \operatorname{Irr}(\tilde{L})^{\tilde{H}}$ such that $A \leq \operatorname{Ker}(\operatorname{det}(\tilde{\gamma}))$. Then $\bar{\gamma}=\operatorname{Res}_{L}^{K}(\bar{\theta})$ is the unique irreducible character of $(k L) \bar{b}$ and $\overline{\tilde{\gamma}}$ is an $\tilde{H}$-stable irreducible character of $k \tilde{L}$ that extends $\bar{\gamma}$. As in [14, Lemma 3.2], since $\tilde{H}=\tilde{L} H$, 3, Theorem 4.4] implies that the "Clifford extensions" of $k H$ with respect to $\bar{\gamma}=\operatorname{Res}_{L}^{K}(\bar{\theta})$ and of $k \tilde{H}$ with respect to $\overline{\tilde{\gamma}}$ are isomorphic twisted $H_{1} / L_{1}$-graded $k$-algebras.

Note that $C_{H}(A)=H_{1}$ and let $\tilde{H}_{1}=H_{1} \times A$ and $\tilde{L}_{1}=L_{1} \times A$, so that $L_{1} \unlhd \tilde{H}_{1}$ and $\tilde{L}_{1} \unlhd \tilde{H}_{1}$. Set $\delta=\operatorname{Res}_{L_{1}}^{K_{1}}\left(\theta_{1}\right) \in \operatorname{Irr}\left(L_{1}\right)^{\tilde{H}_{1}}$. Then $\delta$ has $q$ different extensions to irreducible characters $\delta \times \lambda \in \operatorname{Irr}\left(\tilde{L}_{1}\right)$ for all $\lambda \in \operatorname{Irr}(A)$. Similarly $\bar{\delta}$ is the unique irreducible character of $k L_{1} \overline{W(b)}$ and $\overline{\delta \times \lambda}$ is an $\tilde{H}_{1}$-stable irreducible character of $k \tilde{L}_{1}$ that extends $\bar{\delta}$ for each $\lambda \in \operatorname{Irr}(A)$.

As above, the "Clifford extensions" of $k H_{1}$ with respect to $\bar{\delta}$ and of $k \tilde{H}_{1}$ with respect to $\overline{\delta \times \lambda}$ are isomorphic twisted $H_{1} / L_{1}$-graded $k$-algebras for each $\lambda \in$ $\operatorname{Irr}(A)$.

Clearly $\gamma=\operatorname{Res}_{L}^{K}(\theta) \in \operatorname{Irr}(b)$, where $b \in B \ell(L)$ has defect group $Z(D)$ and $Z(D) \leq \operatorname{Ker}(\gamma)$. Also $\tilde{\gamma}$ lies in a block of $\tilde{L}$ with defect group $Z(D)$ that covers $b$ and $Z(D) \leq \operatorname{Ker}(\tilde{\gamma})$. Similarly $\delta=\operatorname{Res}_{L_{1}}^{K_{1}}\left(\theta_{1}\right) \in \operatorname{Irr}(W(b))$, where $W(b) \in B \ell\left(L_{1}\right)$ has defect group $Z(D), Z(D) \leq \operatorname{Ker}(\delta)$ and $\delta \times \lambda$ lies in a block of $\tilde{L}_{1}$ with defect group $Z(D)$ that covers $W(b)$ and $Z(D) \leq \operatorname{Ker}(\delta \times \lambda)$ for all $\lambda \in \operatorname{Irr}(A)$.

Fix $\lambda \in \operatorname{Irr}(A)$. 
We may view $\tilde{\gamma}$ and $\delta \times \lambda$ as elements of $\operatorname{Irr}(\tilde{L} / Z(D))$ and $\operatorname{Irr}\left(\tilde{L}_{1} / Z(D)\right)$, resp.; in which case we have

$$
\left(\operatorname{Res}_{\tilde{L}_{1}}^{\tilde{L}}(\tilde{\gamma}), \delta \times \lambda\right)_{\tilde{L}_{1}}=\left(\operatorname{Res}_{\tilde{L}_{1} / Z(D)}^{\tilde{L} / Z(D)}(\tilde{\gamma}), \delta \times \lambda\right)_{\tilde{L}_{1} / Z(D)} .
$$

Let $\tilde{V}$ be an $\mathcal{O}$-free indecomposable $\mathcal{O} \tilde{L}$-module that affords $\tilde{\gamma}$ and let $\tilde{W}$ be an $\mathcal{O}$-free indecomposable $\mathcal{O} \tilde{L}_{1}$-module that affords $\delta \times \lambda$. Here $Z(D) \leq \operatorname{Ker}(\tilde{V})$ and $\tilde{V}$ lies in a block of $\tilde{L}$ with defect group $Z(D)$ so that $\overline{\tilde{V}}$ is an irreducible $k \tilde{L}$-module with character $\overline{\tilde{\gamma}}$. Moreover, we may view $\overline{\tilde{V}}$ as an irreducible $k(\tilde{L} / Z(D))$-module that lies in a block of defect 0 of $\tilde{L} / Z(D)$. Similarly, $Z(D) \leq \operatorname{Ker}(\tilde{W})$ and $\tilde{W}$ lies in a block of $\tilde{L}_{1}$ with defect group $Z(D)$ so that $\overline{\tilde{W}}$ is an irreducible $k \tilde{L}_{1}$-module with character $\overline{\delta \times \lambda}$. Clearly we may view $\bar{W}$ as an irreducible $k\left(L_{1} / Z(D)\right)$-module that lies in a block of defect 0 of $L_{1} / Z(D)$. Here the multiplcity $\omega$ of $\overline{\tilde{W}}$ as a $k \tilde{L}_{1}$-module composition factor of $\left.\operatorname{Res}_{\tilde{L}_{1}} \tilde{L}_{(\bar{V}}\right)$ is the same as the multiplicity of $\overline{\tilde{W}}$ as a $k\left(\tilde{L}_{1} / Z(D)\right)$-module composition factor of $\operatorname{Res}_{\tilde{L}_{1} / Z(D)}^{\tilde{L} / Z(D)}(\overline{\tilde{V}})$. Now Lemma 1.18 and (2.1) imply that $\omega=\left(\operatorname{Res}_{\tilde{L}_{1}}^{\tilde{L}}(\tilde{\gamma}), \delta \times \lambda\right)_{\tilde{L}_{1}}$. Consequently, the proof of [14, Lemma 3.2] implies that we may choose $w_{t}^{\prime} \in\left(\left(\mathcal{O} L_{1}\right) W(b)\right)^{\times}$for each $t \in \mathcal{T}$, where $w_{1}^{\prime}=W(b)$ such that, setting $v_{t}^{\prime}=t W(b) w_{t}^{\prime}$ for all $t \in \mathcal{T}$, we have

$$
C_{(\mathcal{O} C) W(b)}\left(\mathcal{S}_{1}\right)=\oplus_{t \in \mathcal{T}}\left(((\mathcal{O} D) W(b)) v_{t}^{\prime}\right),
$$

$v_{t}^{\prime} \alpha v_{t}^{\prime}=t \alpha t^{-1}={ }^{t} \alpha$ for all $\alpha \in(\mathcal{O} D) W(b)$, and such that there is an $\mathcal{O}$ algebra isomorphism $\Phi: C_{(\mathcal{O} C) W(b)}\left(\mathcal{S}_{1}\right)=\oplus_{t \in \mathcal{T}}\left(((\mathcal{O} D) W(b)) v_{t}^{\prime}\right) \rightarrow C_{(\mathcal{O} G) b}(\mathcal{S})=$ $\oplus_{t \in \mathcal{T}}\left(((\mathcal{O} D) b) v_{t}\right)$ such that $(\alpha W(b)) v_{t}^{\prime} \mapsto(\alpha b) v_{t}$ for all $\alpha \in \mathcal{O} D$ and all $t \in \mathcal{T}$. Consequently with this choice, we have $c^{\prime}\left(t_{1} L_{1}, t_{2} L_{1}\right)=c\left(t_{1} L_{1}, t_{2} L_{1}\right)$ for all $t_{1}, t_{2} \in \mathcal{T}$. Now Propositions 2.1(e), (f) and 2.3(e), (f) imply that $\mathcal{O} G b$-mod and $\mathcal{O} C W(b)$-mod are Morita equivalent.

Let $\mathcal{S}=\operatorname{End}_{\mathcal{O}}(U)$ and $\mathcal{S}_{1}=\operatorname{End}_{\mathcal{O}}\left(U_{1}\right)$, where $U, U_{1}$ are $\mathcal{O}$-free modules of ranks $r, r_{1}$, respectively. Then, as in [8] Section 4], since $\mu: \mathcal{S} \otimes_{\mathcal{O}} C_{(\mathcal{O} G) b}(\mathcal{S}) \rightarrow(\mathcal{O} G) b$ and $\mu_{1}: \mathcal{S}_{1} \otimes C_{(\mathcal{O} C) W(b)}\left(\mathcal{S}_{1}\right) \rightarrow(\mathcal{O} C) W(b)$ of Propositions 2.1(f) and 2.3(f) are $\mathcal{O}$ algebra isomorphisms, the $(\mathcal{O} G) b$-mod- $(\mathcal{O} C) W(b)$ bimodule $M$ inducing the Morita equivalence above is given explicitly by

$$
M=\left(U \otimes_{\mathcal{O}}\left(C_{(\mathcal{O} G) b}(\mathcal{S})_{\Phi}\right)\right) \otimes_{C_{(\mathcal{O C}) W(b)}\left(\mathcal{S}_{1}\right)}\left(C_{(\mathcal{O} C) W(b)}\left(\mathcal{S}_{1}\right) \otimes_{\mathcal{O}} U_{1}^{*}\right),
$$

where $\Phi: C_{(\mathcal{O} C) W(b)}\left(\mathcal{S}_{1}\right) \rightarrow C_{(\mathcal{O} G) b}(\mathcal{S})$ is the $\mathcal{O}$-algebra isomorphism above and $U_{1}^{*}=\operatorname{Hom}_{\mathcal{O}}\left(U_{1}, \mathcal{O}\right)$ is the dual module of $U_{1}$.

Thus $M \cong U \otimes_{\mathcal{O}}\left(C_{(\mathcal{O} G) b}(\mathcal{S})_{\Phi}\right) \otimes_{\mathcal{O}} U_{1}^{*}$ in $(\mathcal{O} G) b$-mod- $(\mathcal{O} C) W(b)$.

Using the method of [8, Section 4], we show that the indecomposible $(\mathcal{O} G) \otimes_{\mathcal{O}}$ $\left(\mathcal{O} C\right.$ )-module $\mathcal{M}=U \otimes_{\mathcal{O}}\left(C_{(\mathcal{O} G) b}(\mathcal{S})_{\Phi}\right) \otimes_{\mathcal{O}} U_{1}^{*}$ has $\Delta D$ as a vertex and a trivial source.

Here $D \times D$ is the defect group of the block corresponding to $b \otimes_{\mathcal{O}} W(b)$ in $(\mathcal{O} G) \otimes_{\mathcal{O}}(\mathcal{O} C) \stackrel{\sim}{=}(G \times C)$. Thus $\mathcal{M}$, viewed in $(\mathcal{O} G) \otimes_{\mathcal{O}}(\mathcal{O} C)$-mod, is $D \times D$ projective. Following [8] Section 4] and noting that $D \unlhd G$, that the isomorphism $\mu: \mathcal{S} \otimes C_{(\mathcal{O} G) b}(\mathcal{S}) \rightarrow(\mathcal{O} G) b$ sends $b \otimes \mathcal{O} d b \rightarrow d b$ for all $d \in D$ and that the isomorphism $\mu_{1}: \mathcal{S}_{1} \otimes_{\mathcal{O}} C_{(\mathcal{O} C) W(b)}\left(\mathcal{S}_{1}\right) \rightarrow(\mathcal{O} C) W(b)$ sends $W(b) \otimes d W(b) \mapsto d W(b)$ for all $d \in D$, we observe that the restriction of $\mathcal{M}$ to $D \times D$ is isomorphic to a direct sum of the modules $((\mathcal{O} D) b) v_{t}$ for all $t \in \mathcal{T}$. Here $(\mathcal{O} D) t \cong((\mathcal{O} D) b) v_{t}$ in $\mathcal{O}(D \times D)$-mod and so $((\mathcal{O} D) b) v_{t} \cong \operatorname{Ind}_{R_{t}}^{\mathcal{O}(D \times D)}(\mathcal{O})$ in $\mathcal{O}(D \times D)$-mod, where 
$R_{t}=\left\{\left(d, t^{-1} d t\right) \mid d \in D\right\}$ for each $t \in \mathcal{T}$. Thus [5, III, Lemma 4.6] implies that $\mathcal{M}$ has $\Delta D$ as a vertex and a trivial source.

Now we proceed to demonstrate that such a Morita equivalence can be chosen so as to satisfy (ii) of Theorem 2.

Let $\mathcal{I}((\mathcal{O} D) b)=\sum_{d \in D^{\#}} \mathcal{O}(d-1) b$ and $\mathcal{I}((\mathcal{O} Z(D)) b)=\sum_{d \in Z(D) \#} \mathcal{O}(d-1) b$ denote the augmentation ideals of $(\mathcal{O} D) b$ and $(\mathcal{O} Z(D)) b$, resp. as in Lemma 1.14. Set $\mathcal{I}((\mathcal{O} L) b)=(\mathcal{O} L) b \mathcal{I}((\mathcal{O} Z(D)) b)$ and $\mathcal{I}((\mathcal{O} K) b)=(\mathcal{O} K) b \mathcal{I}((\mathcal{O} D) b)$. Then $\mathcal{I}((\mathcal{O} L) b)=\mathcal{S I}(\mathcal{O} Z(D) b)=\mathcal{I}(\mathcal{O} Z(D) b) \mathcal{S}, \mathcal{I}((\mathcal{O} L) b)$ is an ideal of $(\mathcal{O} L) b$, $\mathcal{I}((\mathcal{O} L) b) \leq J((\mathcal{O} L) b)$, and $\mathcal{I}((\mathcal{O} L) b)=\oplus_{d \in Z(D) \#} \mathcal{S}(d-1)$ and $(\mathcal{O} L) b=\mathcal{S} \oplus$ $\mathcal{I}((\mathcal{O} L) b)$ in $\mathcal{S}$-mod- $\mathcal{S}$. Thus the $\mathcal{O}$-algebra projection $\pi_{\mathcal{S}}:(\mathcal{O} L) b=\mathcal{S} \oplus \mathcal{I}((\mathcal{O} L) b) \rightarrow$ $\mathcal{S}$ in $\mathcal{S}$-mod-S is an epimorphism with $\operatorname{Ker}\left(\pi_{\mathcal{S}}\right)=\mathcal{I}((\mathcal{O} L) b)$. Moreover Proposition 2.1(c) implies that $C_{(\mathcal{O} L) b}(\mathcal{S})=\mathcal{O} Z(D) b$.

Similar facts hold for $(\mathcal{O} K) b: \mathcal{I}((\mathcal{O} K) b)=\mathcal{S I}((\mathcal{O} D) b)=\mathcal{I}((\mathcal{O} D) b) \mathcal{S}, \mathcal{I}((\mathcal{O} K) b)$ is an ideal of $(\mathcal{O} K) b, \mathcal{I}((\mathcal{O} K) b) \leq J((\mathcal{O} K) b), \mathcal{I}((\mathcal{O} K) b)=\oplus_{d \in D^{\#} \mathcal{S}(d-1) b \text { and }}$ $(\mathcal{O} K) b=\mathcal{S} \oplus \mathcal{I}((\mathcal{O} K) b)$ in $\mathcal{S}$-mod-S . Also the projection $\pi_{\mathcal{S}}:(\mathcal{O} K) b=\mathcal{S} \oplus$ $\mathcal{I}((\mathcal{O} K) b) \rightarrow \mathcal{S}$ in $\mathcal{S}$-mod- $\mathcal{S}$ is an epimorphism with $\operatorname{Ker}\left(\pi_{\mathcal{S}}\right)=\mathcal{I}((\mathcal{O} K) b)$ and $C_{(\mathcal{O} K) b}(\mathcal{S})=(\mathcal{O} D) b$.

Similarly, we define $\mathcal{I}((\mathcal{O} D) W(b)), \quad \mathcal{I}((\mathcal{O} Z(D)) W(b)), \quad \mathcal{I}\left(\left(\mathcal{O} L_{1}\right) W(b)\right)$, $\mathcal{I}\left(\left(\mathcal{O} K_{1}\right) W(b)\right), \pi_{\mathcal{S}_{1}}:\left(\mathcal{O} L_{1}\right) W(b) \rightarrow \mathcal{S}_{1}$ and $\pi_{\mathcal{S}_{1}}:\left(\mathcal{O} K_{1}\right) W(b) \rightarrow \mathcal{S}_{1}$ and we have the corresponding facts.

Extending to $\mathcal{K}$, it is clear that

$$
\begin{aligned}
\theta & =T r_{\mathcal{S}} \circ \pi_{\mathcal{S}}:(\mathcal{O} K) b \rightarrow \mathcal{O} \text { and } \\
\theta_{1} & =T r_{\mathcal{S}_{1}} \circ \pi_{\mathcal{S}_{1}}:\left(\mathcal{O} K_{1}\right) W(b) \rightarrow \mathcal{O}
\end{aligned}
$$

canonically induce irreducible characters of $K$ and $K_{1}$, respectively, with $D$ contained in their kernels and such that $\theta \in \operatorname{Irr}_{\mathcal{K}}(b)$ and $\theta_{1} \in \operatorname{Irr}_{\mathcal{K}}(W(b))$.

Lemma 2.5. (a) $b+\mathcal{I}((\mathcal{O} L) b) \unlhd((\mathcal{O} L) b)^{\times},((\mathcal{O} L) b)^{\times}=\mathcal{S}^{\times}(b+\mathcal{I}((\mathcal{O} L) b))=$ $(b+\mathcal{I}((\mathcal{O} L) b)) \mathcal{S}^{\times}$and $\mathcal{S}^{\times} \cap(b+\mathcal{I}((\mathcal{O} L) b))=b ;$

(b)

$$
N_{((\mathcal{O} L) b) \times}\left(\mathcal{S}^{\times}\right)=\mathcal{S}^{\times} \times(b+\mathcal{I}(\mathcal{O} Z(D) b))
$$

and

$$
N_{b+\mathcal{I}((\mathcal{O} L) b)}\left(\mathcal{S}^{\times}\right)=C_{b+\mathcal{I}((\mathcal{O} L) b)}\left(\mathcal{S}^{\times}\right)=b+\mathcal{I}(\mathcal{O} Z(D) b) .
$$

Similar results hold for $(\mathcal{O} K) b$ with $(\mathcal{O D}) b$ in place of $\mathcal{O} Z(D) b$.

Proof. Clearly $b+\mathcal{I}((\mathcal{O} L) b) \subseteq b+J((\mathcal{O} L) b) \unlhd((\mathcal{O} L) b)^{\times}$. Let $i \in \mathcal{I}((\mathcal{O} L) b)$. Then there is an element $j \in J((\mathcal{O} L) b)$ such that $(b+i)(b+j)=b$. Thus $j=-i-i j \in$ $\mathcal{I}((\mathcal{O} L) b)$ and hence $b+\mathcal{I}((\mathcal{O} L) b) \unlhd((\mathcal{O} L) b)^{\times}$. Let $x \in((\mathcal{O} L) b)^{\times}$, so that $x=s+i$ for some $s \in \mathcal{S}$ and $i \in \mathcal{I}((\mathcal{O} L) b)$. The projection $\pi_{\mathcal{S}}:(\mathcal{O} L) b \rightarrow \mathcal{S}$ yields an element $t \in \mathcal{S}$ such that $s t=t s=b$. Thus $x=s t x=s(b+t i) \in \mathcal{S}^{\times}(b+\mathcal{I}((\mathcal{O} L) b))$ and, since $\pi_{\mathcal{S}}(x)=s$, (a) holds. For (b), note that $\mathcal{S}^{\times} \unlhd N_{((\mathcal{O} L) b) \times}\left(\mathcal{S}^{\times}\right)=$ $\mathcal{S}^{\times} N_{b+\mathcal{I}((\mathcal{O} L) b)}\left(\mathcal{S}^{\times}\right)$and $N_{b+\mathcal{I}((\mathcal{O} L) b)}\left(\mathcal{S}^{\times}\right) \unlhd N_{((\mathcal{O} L) b) \times}\left(\mathcal{S}^{\times}\right)$. Thus

$$
\left[\mathcal{S}^{\times}, N_{b+\mathcal{I}(\mathcal{O} L) b)}\left(\mathcal{S}^{\times}\right)\right] \subseteq \mathcal{S}^{\times} \cap(b+\mathcal{I}((\mathcal{O} L) b))=b
$$

and (b) follows from Propositions 2.1(c) and 1.12(e).

Corollary 2.6. $b+J((\mathcal{O} L) b)=(b+J(\mathcal{S}))(b+\mathcal{I}((\mathcal{O} L) b))=(b+\mathcal{I}((\mathcal{O} L) b))(b+J(\mathcal{S}))$. A similar result holds for $(\mathcal{O} K) b$. 
Proof. Since $b+J((\mathcal{O} L) b)=\left((b+J((\mathcal{O} L) b)) \cap \mathcal{S}^{\times}\right)(b+\mathcal{I}((\mathcal{O} L) b))$ by Lemma 2.5 (a) and $(b+J((\mathcal{O} L) b)) \cap \mathcal{S}^{\times}=b+J(\mathcal{S})$ by Proposition $1.12($ a $)$, we are done.

Lemma 2.7. (a) If $x \in((\mathcal{O} G) b)^{\times}$, acting by conjugation, normalizes $(\mathcal{O} L) b$ and $\mathcal{I}((\mathcal{O} L) b)$, and if $u \in(\mathcal{O} L) b$, then $\left.{ }^{x} \pi_{\mathcal{S}}(u)=\pi_{x_{\mathcal{S}}}{ }^{x} u\right)$; and

(b) if $x \in b+\mathcal{I}((\mathcal{O} L) b)$ and $u \in(\mathcal{O} L) b$, then $x^{-1} \in b+\mathcal{I}((\mathcal{O} L) b)$ and $\pi_{\mathcal{S}}\left({ }^{x} u\right)=$ $\pi_{\mathcal{S}}(u)$. Similar results hold for $(\mathcal{O} K) b$.

Proof. Assume the conditions of (a) so that $u=s+i$ for unique $s \in \mathcal{S}$ and $i \in \mathcal{I}((\mathcal{O} L) b)$. Then ${ }^{x} u={ }^{x} s+{ }^{x} i$, so that $\pi_{x_{\mathcal{S}}}\left({ }^{x} u\right)={ }^{x} s={ }^{x} \pi_{\mathcal{S}}(u)$. Assume the conditions of (b) so that, $x^{-1} \in b+\mathcal{I}((\mathcal{O} L) b)$ by Lemma 2.5(a) and $\pi_{\mathcal{S}}\left({ }^{x} u\right)=$ $\pi_{\mathcal{S}}\left(x u x^{-1}\right)=\pi_{\mathcal{S}}(x) \pi_{\mathcal{S}}(u) \pi_{\mathcal{S}}\left(x^{-1}\right)=\pi_{\mathcal{S}}(u)$ and we are done.

Remark 2.8. If $g \in G$, then every element of $((\mathcal{O} L) b)^{\times}(g b)$ normalizes both $(\mathcal{O} L) b$ and $\mathcal{I}((\mathcal{O} L) b)$.

Let $\Sigma$ denote the set of maximal $\mathcal{O}$-semi-simple $\mathcal{O}$-subalgebras of $(\mathcal{O} L) b$. Here $\mathcal{S} \in \Sigma$, all elements of $\Sigma$ are $\mathcal{O}$-simple and $(\mathcal{O} L) b=\mathcal{S}^{\times}(b+\mathcal{I}((\mathcal{O} L) b))$. Thus $b+\mathcal{I}((\mathcal{O} L) b)$ acts transitively on $\Sigma$ by conjugation ([18, Lemma 45.6]).

Lemma 2.9. Let $X$ be a finite subgroup of $A u t_{\mathcal{O}}((\mathcal{O} L) b)$ of order prime to $p$ that leaves $\mathcal{I}((\mathcal{O} L) b)$ invariant. Then there is an $x \in b+\mathcal{I}((\mathcal{O} L) b)$ such that ${ }^{x} \mathcal{S}$ is $X$-invariant.

Proof. Set $\mathcal{G}=(b+J((\mathcal{O} L) b)) \rtimes X$, so that $\mathcal{G}$ permutes $\Sigma$. Since $b+\mathcal{I}((\mathcal{O} L) b)$ and $b+$ $J((\mathcal{O} L) b)$ are transitive on $\Sigma, \mathcal{G}=(b+J((\mathcal{O} L) b)) N_{\mathcal{G}}\left(\mathcal{S}^{\times}\right)=(b+\mathcal{I}((\mathcal{O} L) b)) N_{\mathcal{G}}\left(\mathcal{S}^{\times}\right)$ since $b+\mathcal{I}((\mathcal{O} L) b) \unlhd \mathcal{G}$ by Lemma 2.5. Applying [18, Lemma 45.6], it suffices to prove that $N_{b+J((\mathcal{O} L) b)}\left(\mathcal{S}^{\times}\right)$has a complement in $N_{\mathcal{G}}\left(\mathcal{S}^{\times}\right)$. Here $N_{b+J((\mathcal{O} L) b)}\left(\mathcal{S}^{\times}\right)=$ $N_{b+J((\mathcal{O} L) b)}(\mathcal{S})=(b+\mathcal{I}(\mathcal{O} Z(D) b)) \times(b+J(\mathcal{S}))$ by Lemma $2.5(\mathrm{~b})$ and Lemma 1.5 (b). Also $b+J(\mathcal{S})$ and $b+\mathcal{I}(\mathcal{O} Z(D) b)=N_{b+\mathcal{I}((\mathcal{O} L) b)}\left(\mathcal{S}^{\times}\right)$are normal subgroups of $N_{\mathcal{G}}\left(\mathcal{S}^{\times}\right)$and $N_{\mathcal{G}}\left(\mathcal{S}^{\times}\right) / N_{b+J((\mathcal{O} L) b)}\left(\mathcal{S}^{\times}\right) \simeq X$. Set

$$
\overline{N_{\mathcal{G}}\left(\mathcal{S}^{\times}\right)}=N_{\mathcal{G}}\left(\mathcal{S}^{\times}\right) /(b+\mathcal{I}(\mathcal{O} Z(D) b)) .
$$

Then $b+J(\mathcal{S}) \tilde{=} \overline{b+J(\mathcal{S})} \unlhd \overline{N_{\mathcal{G}}\left(\mathcal{S}^{\times}\right)}$and [18, Lemma 45.6] implies that $\overline{b+J(\mathcal{S})}$ has a complement $\bar{Y}$ in $\overline{N_{\mathcal{G}}\left(\mathcal{S}^{\times}\right)}$. The inverse image $Y$ of $\bar{Y}$ in $N_{\mathcal{G}}\left(\mathcal{S}^{\times}\right)$satisfies $b+\mathcal{I}(\mathcal{O} Z(D) b) \leq Y$ and $Y /(b+\mathcal{I}(\mathcal{O} Z(D) b)) \tilde{=} X$. Since $b+\mathcal{I}(\mathcal{O} Z(D) b)$ is an Abelian group, 9, I, Hauptsatz 17.4] and Lemma 1.14(b) imply that $b+\mathcal{I}(\mathcal{O} Z(D) b)$ has a complement $B$ in $Y$. Clearly $B$ is a complement to $N_{b+J((\mathcal{O} L) b)}\left(\mathcal{S}^{\times}\right)$in $N_{\mathcal{G}}\left(\mathcal{S}^{\times}\right)$ and we are done.

Corollary 2.10. A leaves invariant an element of $\Sigma$.

We shall henceforth assume that $\mathcal{S} \in \Sigma$ is $A$-invariant. It follows that $\pi_{\mathcal{S}}$ : $(\mathcal{O} L) b \rightarrow \mathcal{S}$ and $\pi_{\mathcal{S}}:(\mathcal{O} K) b \rightarrow \mathcal{S}$ are $A$-projections and that $C_{(\mathcal{O} H) b}(\mathcal{S})=$ $\oplus_{t \in \mathcal{T}}(\mathcal{O} Z(D) b) v_{t}$ and $C_{(\mathcal{O} G) b}(\mathcal{S})=\oplus_{t \in \mathcal{T}}((\mathcal{O} D) b) v_{t}$ are $A$-invariant. In fact, for each $t \in \mathcal{T}, C_{((\mathcal{O} L) b)(t b)}(\mathcal{S})=C_{((\mathcal{O} L) b) v_{t}}(\mathcal{S})=\mathcal{O} Z(D) b v_{t}$ and $C_{(\mathcal{O} G) b(t b)}(\mathcal{S})=$ $(\mathcal{O D}) b v_{t}$ are $A$-invariant and, from Proposition 2.1(e), $v_{t}=w_{t}(t b)$ for some $w_{t} \in$ $((\mathcal{O} L) b)^{\times}$. Consequently $w_{t}=s_{t} \alpha_{t}$ for unique $s_{t} \in \mathcal{S}^{\times}$and $\alpha_{t} \in b+\mathcal{I}((\mathcal{O} L) b)$ by Lemma 2.5(a) for each $t \in \mathcal{T}$. Since $w_{1}=b$, we have $s_{1}=\alpha_{1}=b$.

Lemma 2.11. (a) A acts trivially on $C_{(\mathcal{O} H) b}(\mathcal{S})=\oplus_{t \in \mathcal{T}} \mathcal{O} Z(D) b v_{t}$ and $C_{(\mathcal{O} G) b}(\mathcal{S})$ $=\oplus_{t \in \mathcal{T}}(\mathcal{O} D) b v_{t} ;$ and

(b) $s_{t}$ and $\alpha_{t}$ are fixed by $A$ for all $t \in \mathcal{T}$. 
Proof. Let $t \in \mathcal{T}$ and $a \in A$. Then $v_{t}^{a}=\alpha v_{t}$ for a unique $\alpha \in \mathcal{O} Z(D) b$. Hence $\alpha^{|a|}=b$. Since $(|a|, p)=1$, Lemma 1.13(a) and Proposition 1.8 imply that $\alpha=\gamma b$ for a unique $\gamma \in \mathcal{O}^{\times}$such that $\gamma^{|a|}=1$. However $v_{t}^{m}=\delta b$ for a unique $\delta \in \Omega \leq$ $\left\{x \in \mathcal{O}^{\times} \mid x^{m}=1\right\}$ by Proposition 2.1. Hence $\left(v_{t}^{a}\right)^{m}=\gamma^{m}(\delta b)=(\delta b)^{a}=\delta b$ so that $\gamma^{m}=1$. As $(|a|, m)=1$, we conclude that $\gamma=1$ and (a) follows. Since $v_{t}=s_{t} \alpha_{t}(t b)$ for unique $s_{t} \in \mathcal{S}^{\times}$and $\alpha_{t} \in b+\mathcal{I}((\mathcal{O} L) b)$ and both $\mathcal{S}^{\times}$and $b+\mathcal{I}((\mathcal{O} L) b)$ are $A$ invariant, Lemma 2.5(a) implies (b). Our proof is complete.

Recall that $G=\bigcup_{t \in \mathcal{T}} K t$, where the union is disjoint. We define $\pi_{\mathcal{S}}^{*}: G \rightarrow \mathcal{S}^{\times}$ by $k t \mapsto \pi_{\mathcal{S}}(k b) s_{t}^{-1}$ for all $k \in K$ and all $t \in \mathcal{T}$ and we extend $\pi_{\mathcal{S}}^{*}$ to an $\mathcal{O}$-linear map $\pi_{\mathcal{S}}^{*}: \mathcal{O} G \rightarrow \mathcal{S}$. Since $s_{1}=b, \pi_{\mathcal{S}}^{*}$ extends the $\mathcal{O}$-algebra $A$-epimorphism $\pi_{\mathcal{S}}:(\mathcal{O} K) b \rightarrow \mathcal{S}$. Clearly $\pi_{\mathcal{S}}^{*}(\alpha)=\pi_{\mathcal{S}}^{*}(\alpha b)$ for all $\alpha \in \mathcal{O} G$.

Lemma 2.12. Let $\alpha, \beta \in \mathcal{O} K$ and $t \in \mathcal{T}$. Then:

(a) $\pi_{\mathcal{S}}^{*}(\alpha) \pi_{\mathcal{S}}^{*}(\beta t)=\pi_{\mathcal{S}}((\alpha \beta) b) s_{t}^{-1}=\pi_{\mathcal{S}}^{*}(\alpha \beta t)$;

(b) $\pi_{\mathcal{S}}^{*}(\alpha t \beta)=\pi_{\mathcal{S}}^{*}\left(\alpha\left({ }^{t} \beta\right) t\right)=\pi_{\mathcal{S}}\left(\alpha\left({ }^{t} \beta\right) b\right) s_{t}^{-1}=\pi_{\mathcal{S}}^{*}(\alpha) \pi_{\mathcal{S}}^{*}\left(\left({ }^{t} \beta\right) t\right)=\pi_{\mathcal{S}}^{*}(\alpha) \pi_{\mathcal{S}}^{*}(t \beta)$;

(c) $\pi_{\mathcal{S}}^{*}(1)=b=\pi_{\mathcal{S}}^{*}(b), \pi_{\mathcal{S}}^{*}((1-b) \mathcal{O} G)=\pi_{\mathcal{S}}^{*}(\mathcal{O} G(1-b))=0$ and $\pi_{\mathcal{S}}^{*}:(\mathcal{O} G) b \rightarrow \mathcal{S}$ is a surjective $\mathcal{O}$-linear map; and

(d) $\pi_{\mathcal{S}}^{*}\left(v_{t}\right)=b$ and $s_{t}^{-1} \pi_{\mathcal{S}}(\beta b) s_{t}=\pi_{\mathcal{S}}\left({ }^{t}(\beta b)\right)$.

Proof. Clearly (a)-(c) hold. Since $v_{t}=s_{t} \alpha_{t}(t b)$, we have $\pi_{\mathcal{S}}^{*}\left(v_{t}\right)=\pi_{\mathcal{S}}\left(s_{t} \alpha_{t}\right) s_{t}^{-1}$ $=s_{t} s_{t}^{-1}=b$. Also $s_{t}^{-1} v_{t}=\alpha_{t}(t b) \in N_{((\mathcal{O} G) b) \times}(\mathcal{S})$. Thus

$$
s_{t}^{-1} \pi_{\mathcal{S}}(\beta b) s_{t}=s_{t}^{-1} v_{t} \pi_{\mathcal{S}}(\beta b) v_{t}^{-1} s_{t}=\pi_{\mathcal{S}}\left({ }^{\alpha_{t}(t b)}(\beta b)\right)=\pi_{\mathcal{S}}\left({ }^{t b}(\beta b)\right)=\pi_{\mathcal{S}}\left({ }^{t}(\beta b)\right)
$$

using Lemma 2.7, the fact that $\alpha_{t}$ and $t b$ normalize $(\mathcal{O} L) b$ and $\mathcal{I}((\mathcal{O} L) b)$ and the fact that $\alpha_{t} \in \operatorname{Ker}\left(\pi_{\mathcal{S}}\right)$. Our proof is complete.

Lemma 2.13. Let $k_{1}, k_{2} \in K$ and $t_{1}, t_{2} \in \mathcal{T}$. Here $t_{1} t_{2}=\ell t_{3}$ and $t_{1}^{-1}=\ell^{\prime} t_{4}$ for unique $\ell, \ell^{\prime} \in L_{1}$ and $t_{3}, t_{4} \in \mathcal{T}$. Then:

(a) $\pi_{\mathcal{S}}^{*}\left(k_{1} t_{1}\right) \pi_{\mathcal{S}}^{*}\left(k_{2} t_{2}\right)=c\left(t_{1} L_{1}, t_{2} L_{1}\right)^{-1} \pi_{\mathcal{S}}^{*}\left(k_{1} t_{1} k_{2} t_{2}\right)$;

(b) $\pi_{\mathcal{S}}^{*}\left(k_{1} t_{1}\right)^{-1}=c\left(t_{1} L_{1}, t_{4} L_{1}\right) \pi_{\mathcal{S}}^{*}\left(\left(k_{1} t_{1}\right)^{-1}\right)$; and

(c) $\pi_{\mathcal{S}}^{*}\left(\left(k_{1} t_{1}\right)^{a}\right)=\pi_{\mathcal{S}}^{*}\left(k_{1}^{a} t_{1}\right)=\pi_{\mathcal{S}}^{*}\left(k_{1}^{a}\right) s_{t_{1}}^{-1}=\pi_{\mathcal{S}}^{*}\left(k_{1} t_{1}\right)^{a}$ for all $a \in A$ and $\pi_{\mathcal{S}}^{*}$ : $\mathcal{O} G \rightarrow \mathcal{S}$ is an $A$-epimorphism in $\mathcal{O}$-mod.

Proof. Clearly $\left(k_{1} t_{1}\right)\left(k_{2} t_{2}\right)=k_{1}\left({ }^{t_{1}} k_{2}\right) \ell t_{3}$ and $\pi_{\mathcal{S}}^{*}\left(k_{1} t_{1} k_{2} t_{2}\right)=\pi_{\mathcal{S}}\left(k_{1}\left({ }^{t_{1}} k_{2}\right) \ell b\right) s_{t_{3}}^{-1}$. Also $\pi_{\mathcal{S}}^{*}\left(k_{1} t_{1}\right) \pi_{\mathcal{S}}^{*}\left(k_{2} t_{2}\right)=\pi_{\mathcal{S}}\left(k_{1} b\right) s_{t_{1}}^{-1} \pi_{\mathcal{S}}\left(k_{2} b\right) s_{t_{2}}^{-1}=\pi_{\mathcal{S}}\left(k_{1} b\right) \pi_{\mathcal{S}}\left(\left({ }^{t_{1}} k_{2}\right) b\right) s_{t_{1}}^{-1} s_{t_{2}}^{-1}=$ $\pi_{\mathcal{S}}\left(k_{1}\left({ }^{t_{1}} k_{2}\right) b\right) s_{t_{1}}^{-1} s_{t_{2}}^{-1}$ using Lemma $2.12(\mathrm{~d})$. Since $v_{t_{1}} v_{t_{2}}=c\left(t_{1} L_{1}, t_{2} L_{2}\right) v_{t_{3}}$, we have $s_{t_{1}}^{-1} v_{t_{1}} s_{t_{2}}^{-1} v_{t_{2}}=s_{t_{1}}^{-1} s_{t_{2}}^{-1} c\left(t_{1} L_{1}, t_{2} L_{1}\right) v_{t_{3}}=\alpha_{t_{1}}\left(t_{1} b\right) \alpha_{t_{2}}\left(t_{2} b\right)=\alpha_{t_{1}}\left({ }^{t_{1}} \alpha_{t_{2}}\right)\left(\ell t_{3}\right) b=$ $\alpha_{t_{1}}\left({ }^{t_{1}} \alpha_{t_{2}}\right)(\ell b) \alpha_{t_{3}}^{-1} s_{t_{3}}^{-1} v_{t_{3}}$. Thus, $c\left(t_{1} L_{1}, t_{2} L_{1}\right) s_{t_{1}}^{-1} s_{t_{2}}^{-1}=\alpha_{t_{1}}\left({ }^{t_{1}} \alpha_{t_{2}}\right)(\ell b) \alpha_{t_{3}}^{-1} s_{t_{3}}^{-1}$ and applying $\pi_{\mathcal{S}}$, we conclude that $c\left(t_{1} L_{1}, t_{2} L_{1}\right) s_{t_{1}}^{-1} s_{t_{2}}^{-1}=\pi_{\mathcal{S}}(\ell b) s_{t_{3}}^{-1}$ and (a) holds. Then (b) and (c) are immediate and we are done.

Recall the $\mathcal{O}$-algebra isomorphism $\Phi: C_{(\mathcal{O} C) W(b)}\left(\mathcal{S}_{1}\right)=\oplus_{t \in \mathcal{T}}\left((\mathcal{O} D) W(b) v_{t}^{\prime}\right) \rightarrow$ $C_{(\mathcal{O} G) b}(\mathcal{S})=\oplus_{t \in \mathcal{T}}(\mathcal{O} D) b v_{t}$ such that $(\alpha W(b)) v_{t}^{\prime} \mapsto(\alpha b) v_{t}$ for all $\alpha \in \mathcal{O} D$ and all $t \in \mathcal{T}$. Thus $v_{t_{1}}^{\prime} v_{t_{2}}^{\prime}=c\left(t_{1} L_{1}, t_{2} L_{1}\right) v_{t_{3}}^{\prime}$, where $t_{1} t_{2} \in L_{1} t_{3}$. For $t \in \mathcal{T}, v_{t}^{\prime}=$ $w_{t}^{\prime}(t W(b))$, where $w_{t}^{\prime} \in\left(\left(\mathcal{O} L_{1}\right) W(b)\right)^{\times}=\mathcal{S}_{1}^{\times}\left(W(b)+\mathcal{I}\left(\left(\mathcal{O} L_{1}\right) W(b)\right)\right)$, so that $w_{t}^{\prime}=$ $s_{t}^{\prime} \alpha_{t}^{\prime}$ for unique $s_{t}^{\prime} \in \mathcal{S}_{1}^{\times}$and $\alpha_{t}^{\prime} \in W(b)+\mathcal{I}\left(\left(\mathcal{O} L_{1}\right) W(b)\right)$. As above, define $\pi_{\mathcal{S}_{1}}^{*}$ : $C=\bigcup_{t \in \mathcal{T}} K_{1} t \rightarrow \mathcal{S}_{1}^{\times}$by $k_{1} t \mapsto \pi_{\mathcal{S}_{1}}\left(k_{1} W(b)\right)\left(s_{t}^{\prime}\right)^{-1}$ for all $k_{1} \in K_{1}$ and $t \in \mathcal{T}$ and extend $\pi_{\mathcal{S}_{1}}^{*}$ to an $\mathcal{O}$-linear map $\pi_{\mathcal{S}_{1}}^{*}: \mathcal{O} C \rightarrow \mathcal{S}_{1}$. The proof of Lemma 2.13 with $C, K_{1}, \mathcal{S}_{1}$ in place of $G, K, \mathcal{S}$ yields the following lemma. 
Lemma 2.14. Let $k_{1}, k_{2} \in K_{1}$ and $t_{1}, t_{2} \in \mathcal{T}$. Here $t_{1} t_{2}=\ell t_{3}$ and $t_{1}^{-1}=\ell^{\prime} t_{4}$ for unique $\ell, \ell^{\prime} \in L_{1}$ and $t_{3}, t_{4} \in \mathcal{T}$. Then:

(a) $\pi_{\mathcal{S}_{1}}^{*}\left(k_{1} t_{1}\right) \pi_{\mathcal{S}_{1}}^{*}\left(k_{2} t_{2}\right)=c\left(t_{1} L_{1}, t_{2} L_{1}\right)^{-1} \pi_{\mathcal{S}_{1}}^{*}\left(k_{1} t_{1} k_{2} t_{2}\right)$;

(b) $\pi_{\mathcal{S}_{1}}^{*}\left(k_{1} t_{1}\right)^{-1}=c\left(t_{1} L_{1}, t_{4} L_{1}\right) \pi_{\mathcal{S}_{1}}^{*}\left(\left(k_{1} t_{1}\right)^{-1}\right)$; and

(c) $\pi_{\mathcal{S}_{1}}^{*}: \mathcal{O} \rightarrow \mathcal{S}_{1}$ is an epimorphism of $\mathcal{O}$-modules.

Observe that $(\mathcal{O} H) b$ can be viewed as an $H / L$-crossed product $\mathcal{O}$-algebra with $((\mathcal{O} H) b)_{t L}=(\mathcal{O} L) b(t b)$ for all $t \in \mathcal{T}$.

Thus Lemma 1.6 yields:

Lemma 2.15. $((\mathcal{O H}) b)^{\times} \cap((\mathcal{O} L) b)=((\mathcal{O} L) b)^{\times}$.

Lemma 2.16. Let $t \in \mathcal{T}$. Then:

(a) if $y \in(K t)_{p^{\prime}}$, then $y$ is $D$-conjugate to an element $u \in(L t)_{p^{\prime}}$; and

(b) if $u \in(L t)_{p^{\prime}}$, then $C_{D}(u)=C_{D}(t), u b \in(\mathcal{O} L) b(t b)=(\mathcal{O} L) v_{t}$ and there is an element $\gamma \in b+\mathcal{I}((\mathcal{O} L) b)$ such that $(u b)^{\gamma}=s v_{t}$, where $s \in \mathcal{S}^{\times}$. Moreover, if $d \in C_{D}(u)$, then $(u d b)^{\gamma}=s\left((d b) v_{t}\right)$ and $\pi_{\mathcal{S}}^{*}(u d b)=s$.

Proof. If $y \in(K t)_{p^{\prime}}$, then, as $G=H D, y$ is $D$-conjugate to an element $u \in$ $(H \cap(K t))_{p^{\prime}}=(L t)_{p^{\prime}}$ and (a) holds. Let $u \in(L t)_{p^{\prime}}$. Then $C_{D}(u)=C_{D}(t)$ and $u b \in((\mathcal{O} L) b) v_{t}$. Set $X=\langle u\rangle$. Then Lemma 2.9 yields an element $z \in b+\mathcal{I}((\mathcal{O} L) b)$ such that $X$ leaves ${ }^{z} \mathcal{S}$ invariant. Consequently $(u b)^{z} \in N_{((\mathcal{O} H) b) \times}(\mathcal{S}) \cap\left((\mathcal{O} L) b v_{t}\right)$. Note that $\left.((\mathcal{O H}) b)^{\times} \cap\left((\mathcal{O} L) b v_{t}\right)=(\mathcal{O} L) b\right)^{\times} v_{t}$ because of Lemma 1.6. Thus $(u b)^{z} \in$ $\left(N_{((\mathcal{O} H) b) \times}(\mathcal{S}) \cap((\mathcal{O} L) b)^{\times}\right) v_{t}=N_{((\mathcal{O} L) b) \times}(\mathcal{S}) v_{t}=\left(\mathcal{S}^{\times} \times(b+\mathcal{I}(\mathcal{O} Z(D) b))\right) v_{t}$ because of Lemma 2.5. Thus $(u b)^{z}=s \alpha v_{t}$ for unique $s \in \mathcal{S}^{\times}$and $\alpha \in b+\mathcal{I}(\mathcal{O} Z(D) b)$. Set $f=|u|$, so that $(f, p)=1$ and $s^{f}\left(\alpha v_{t}\right)^{f}=b$. Consequently, $s^{f} \in \mathcal{S}^{\times} \cap$ $C_{(\mathcal{O} G) b}(\mathcal{S})=\mathcal{O}^{\times} b$ and $s^{f}=\sigma b$ for a unique $\sigma \in \mathcal{O}^{\times}$. Since $(f, p)=1$, there is an element $\delta \in \mathcal{O}^{\times}$such that $\delta^{f}=\sigma$. Then $(u b)^{z}=\left(\delta^{-1} s\right)\left(\alpha\left(\delta v_{t}\right)\right)$, where $\left(\delta^{-1} s\right)^{f}=b=\left(\alpha\left(\delta v_{t}\right)\right)^{f}$. Here $\delta^{f} v_{t}^{f}=\left(\delta v_{t}\right)^{f} \in(b+\mathcal{O} Z(D) b) \cap\left(\oplus_{t \in \mathcal{T}} \mathcal{O} v_{t}\right)=b$. Since $\alpha\left(\delta v_{t}\right) \in(b+J(\mathcal{O} Z(D) b))\left(\delta v_{t}\right) \subseteq(\mathcal{O} Z(D) b)^{\times}\left(\delta v_{t}\right)$ and $v_{t} \alpha v_{t}^{-1}=t \alpha t^{-1}$ for all $\alpha \in(\mathcal{O} D) b$ by Proposition 2.1(e), Lemma 1.11 with $X=(b+J(\mathcal{O} Z(D) b)) E$ where $e=\delta v_{t}$ and $E=\left\langle\delta v_{t}\right\rangle$ and Lemma 1.14(a) imply the existence of an element $x \in b+\mathcal{I}(\mathcal{O} Z(D) b)$ such that $\left(\alpha\left(\delta v_{t}\right)\right)^{x}=\delta v_{t}$. Thus $\gamma=z x \in(b+\mathcal{I}((\mathcal{O} L) b))$ is such that $(u b)^{\gamma}=\left(\delta^{-1} s\right)\left(\alpha\left(\delta v_{t}\right)\right)^{x}=\left(\delta^{-1} s\right)\left(\delta v_{t}\right)=s v_{t}$. If $d \in C_{D}(u)=C_{D}(t)$, then $((u d) b)^{\gamma}=(u b)^{\gamma}(d b)=s\left((d b) v_{t}\right)$. Let $\gamma=b+j$ and $\gamma^{-1}=b+j^{\prime}$, where $j, j^{\prime} \in$ $\mathcal{I}((\mathcal{O} L) b)$. Then $\left(b+j^{\prime}\right)((u d) b)(b+j)=\left(b+j^{\prime}\right)\left(b+{ }^{u} j\right)(u d) b$, where $\left(b+j^{\prime}\right)\left(b+{ }^{u} j\right) \in$ $b+\mathcal{I}((\mathcal{O} L) b)$, so Lemma 2.12 implies that $\pi_{\mathcal{S}}^{*}\left(((u d) b)^{\gamma}\right)=\pi_{\mathcal{S}}^{*}\left(\left(b+j^{\prime}\right)(u d) b(b+j)\right)=$ $\pi_{\mathcal{S}}^{*}((u d) b)=\pi_{\mathcal{S}}^{*}\left(s(d b) v_{t}\right)=s$ and we are done.

We extend $\theta=T r_{\mathcal{S}} \circ \pi_{\mathcal{S}}:(\mathcal{O} K) b \rightarrow \mathcal{O}$ and $\theta_{1}=\operatorname{Tr}_{\mathcal{S}_{1}} \circ \pi_{\mathcal{S}_{1}}:\left(\mathcal{O} K_{1}\right) W(b) \rightarrow \mathcal{O}$ to $\mathcal{O}$-linear maps $\theta=T r_{\mathcal{S}} \circ \pi_{\mathcal{S}}^{*}: \mathcal{O} G \rightarrow \mathcal{O}$ such that $g \mapsto \operatorname{Tr}_{\mathcal{S}}\left(\pi_{\mathcal{S}}^{*}(g b)\right)$ for all $g \in G$ and $\theta_{1}=T r_{\mathcal{S}_{1}} \circ \pi_{\mathcal{S}_{1}}^{*}: \mathcal{O} C \rightarrow \mathcal{O}$ such that $g \mapsto T r_{\mathcal{S}_{1}}\left(\pi_{\mathcal{S}_{1}}^{*}(g W(b))\right)$ for all $g \in C$. Set $\mathcal{B}=C_{(\mathcal{O} G) b}(\mathcal{S})$ and $\mathcal{B}^{\prime}=C_{\mathcal{O} C W(b)}\left(\mathcal{S}_{1}\right)$.

For the remainder of this article we extend coefficients to $\mathcal{K}$ so that $\mathcal{K} \mathcal{S} \simeq M_{r}(\mathcal{K})$, $\mathcal{K} \mathcal{S}_{1} \tilde{=} M_{r_{1}}(\mathcal{K}), \mathcal{K} C_{(\mathcal{O} G) b}(\mathcal{S})=\mathcal{K B}=\bigoplus_{t \in \mathcal{T}}\left(\mathcal{K}(d b) v_{t}\right), \mathcal{K} C_{(\mathcal{O} C) W(b)}\left(\mathcal{S}_{1}\right)=\mathcal{K} \mathcal{B}^{\prime}=$

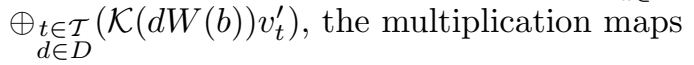

$$
\begin{aligned}
\mu:(\mathcal{K S}) \otimes_{\mathcal{K}}(\mathcal{K} \mathcal{B}) & \rightarrow(\mathcal{K} G) b, \\
\mu_{1}:\left(\mathcal{K} \mathcal{S}_{1}\right) \otimes_{\mathcal{K}}\left(\mathcal{K} \mathcal{B}^{\prime}\right) & \rightarrow(\mathcal{K} C) W(b)
\end{aligned}
$$

are $\mathcal{K}$-algebra isomorphisms, etc. 
Clearly

$$
\operatorname{Irr}_{\mathcal{K}}\left((\mathcal{K S}) \otimes_{\mathcal{K}}(\mathcal{K} \mathcal{B})\right)=\left\{\left(\operatorname{Tr}_{\mathcal{S}} * \psi\right) \mid \psi \in \operatorname{Irr}_{\mathcal{K}}(\mathcal{K} \mathcal{B})\right\},
$$

where $\left(\operatorname{Tr}_{\mathcal{S}} * \psi\right)(s \otimes \mathcal{K} \beta)=\operatorname{Tr}_{\mathcal{S}}(s) \psi(\beta)$ for all $s \in \mathcal{S}$ and $\beta \in \mathcal{B}$ and for all $\psi \in$

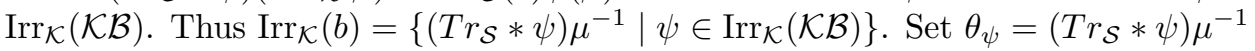
for all $\psi \in \operatorname{Irr}_{\mathcal{K}}(\mathcal{K} \mathcal{B})$. We view $\operatorname{Irr}_{\mathcal{K}}(\mathcal{K} B)$ as a subset of $\operatorname{Irr}_{\mathcal{K}}(\mathcal{K} G)$ in the canonical fashion.

Fix $\psi \in \operatorname{Irr}_{\mathcal{K}}(\mathcal{K} \mathcal{B})$ and $t \in \mathcal{T}$ and let $g \in K t$. Since $D$ is the defect group of $b$, $\theta_{\psi}(g)=0$ if $g_{p} \notin D$ by [5] IV, Lemma 2.4]. Hence, by Lemma 1.22, we have

$$
\theta_{\psi}(g)=0 \text { if } g D \notin(G / D)_{p^{\prime}} .
$$

Suppose that $g D \in(G / D)_{p^{\prime}}$. Then, by Lemma 1.22, $g_{p} \in D$ and $g_{p^{\prime}} \in K t$. Since $G=H D, g_{p^{\prime}}$ is $D$-conjugate to an element $u \in H \cap(K t)=L t$ and $u D=g D \subseteq K t$. By Lemma 2.16, there is an element $\sigma \in b+\mathcal{I}((\mathcal{O} L) b)$ such that $(u b)^{\sigma}=s v_{t}$, where $s \in \mathcal{S}$ and $\pi_{\mathcal{S}}^{*}(g b)=\pi_{\mathcal{S}}^{*}(u b)=s$. Also $C_{D}(u) b=C_{D}(t) b=C_{D b}\left(v_{t}\right)$ since $u \in L t$ and we have $g D=u D=\bigcup_{f \in \mathcal{F}}\left(u C_{D}(u)\right)^{f}$, where $\mathcal{F}$ is a right transversal of $C_{D}(u)$ in $D$ and the union is disjoint. Moreover if $d \in C_{D}(u)$, then $(u d b)^{\sigma}=s\left((d b) v_{t}\right)$ since $\sigma \in b+\mathcal{I}((\mathcal{O} L) b)$. Thus:

if $d \in C_{D}(u)$, then $\theta_{\psi}(u d)=\operatorname{Tr}_{\mathcal{S}}(s) \psi\left((d b) v_{t}\right)$, where $\pi_{\mathcal{S}}^{*}((u d) b)=s$; and

$$
\begin{aligned}
\sum_{d \in D} \theta_{\psi}(u d) \theta_{\psi}\left((u d)^{-1}\right)=\mid D & : C_{D}(t) \mid \operatorname{Tr}_{\mathcal{S}}(s) T r_{\mathcal{S}}\left(s^{-1}\right) \\
& \left.\cdot \sum_{d \in C_{D}(t)}\left(\psi\left((d b) v_{t}\right) \psi\left((d b) v_{t}\right)^{-1}\right)\right) .
\end{aligned}
$$

Remark 2.17. Clearly (2.3) and (2.4) reduce to [16, Theorem 7] (cf. [5, V, Theorem 4.7]) when $G=K$ and present a description of $\operatorname{Irr}_{\mathcal{K}}(b)$ that differs from the description of $\operatorname{Irr}_{\mathcal{K}}(b)$ given in [16, Section 3] and that is consonant with [16, Theorems 5 and 6$]$ and with [15, Theorem A].

Utilizing $\Phi: \mathcal{K} \mathcal{B}^{\prime} \rightarrow \mathcal{K B}$ (the canonic extension of the isomorphism $\Phi: \mathcal{B}^{\prime} \rightarrow \mathcal{B}$ ), we have $\operatorname{Irr}_{\mathcal{K}}\left(\mathcal{K B}^{\prime}\right)=\left\{\psi \Phi \mid \psi \in \operatorname{Irr}_{\mathcal{K}}(\mathcal{K} \mathcal{B})\right\}$.

Clearly $\operatorname{Irr}_{\mathcal{K}}\left(\mathcal{K S}_{1} \otimes_{\mathcal{K}} \mathcal{K} \mathcal{B}^{\prime}\right)=\left\{\operatorname{Tr}_{\mathcal{S}_{1}} *(\psi \Phi) \mid \psi \in \operatorname{Irr}_{\mathcal{K}}(\mathcal{K} \mathcal{B})\right\}$, where $\operatorname{Tr}_{\mathcal{S}_{1}} *(\psi \Phi)$ is as defined above for all $\psi \in \operatorname{Irr}_{\mathcal{K}}(\mathcal{K} \mathcal{B})$. Also $\operatorname{Irr}_{\mathcal{K}}(W(b))=\left\{\left(\operatorname{Tr}_{\mathcal{S}_{1}} *(\psi \Phi)\right) \mu_{1}^{-1} \mid\right.$ $\left.\psi \in \operatorname{Irr}_{\mathcal{K}}(\mathcal{K B})\right\}$ and we set $\theta_{1 \psi}=\left(\operatorname{Tr}_{\mathcal{S}_{1}} *(\psi \Phi)\right) \mu_{1}^{-1}$ for all $\psi \in \operatorname{Irr}_{\mathcal{K}}(\mathcal{K} \mathcal{B})$. Clearly the Morita equivalence above induces the bijection of $\operatorname{Irr}_{\mathcal{K}}(b)$ onto $\operatorname{Irr}_{\mathcal{K}}(W(b))$ that sends $\theta_{\psi} \mapsto \theta_{1 \psi}$ for all $\psi \in \operatorname{Irr}_{\mathcal{K}}(\mathcal{K B})$.

Note that $C_{G / D}(A)=C / D$ and let $g \in K_{1} t$. Then, as above,

$$
\theta_{1 \psi}(g)=0 \text { if } g D \notin(C / D)_{p^{\prime}} .
$$

Suppose that $g D \in(C / D)_{p^{\prime}}$. Then, as above, $g D=u D$ for some $u \in\left(L_{1} t\right) \cap C_{p^{\prime}}$ and $u D=\bigcup_{f \in \mathcal{F}}\left(u C_{D}(u)\right)^{f}$, where $\mathcal{F}$ is a right transversal of $C_{D}(u)$ in $D$ and the union is disjoint. Set $s_{1}=\pi_{\mathcal{S}_{1}}^{*}(u)$, so that $\theta_{1 \psi}(u d)=\operatorname{Tr}_{\mathcal{S}_{1}}\left(s_{1}\right) \psi\left((d b) v_{t}\right)$ for all $d \in C_{D}(u)$. Consequently,

$$
\begin{gathered}
\sum_{d \in D} \theta_{1 \psi}(u d) \theta_{1 \psi}\left((u d)^{-1}\right)=\left|D: C_{D}(t)\right| \operatorname{Tr}_{\mathcal{S}_{1}}\left(s_{1}\right) \operatorname{Tr}_{\mathcal{S}_{1}}\left(s_{1}^{-1}\right) \\
\cdot \sum_{d \in C_{D}(t)}\left(\psi\left((d b) v_{t}\right) \psi\left(\left((d b) v_{t}\right)^{-1}\right)\right)
\end{gathered}
$$


and

$$
\begin{gathered}
\sum_{d \in D} \theta_{\psi}(u d) \theta_{1 \psi}\left((u d)^{-1}\right)=\left|D: C_{D}(t)\right|\left(\operatorname{Tr}_{\mathcal{S}}(s)\right)\left(\operatorname{Tr}_{\mathcal{S}_{1}}\left(s_{1}^{-1}\right)\right) \\
\cdot \sum_{d \in C_{D}(t)}\left(\psi\left((d b) v_{t}\right) \psi\left(\left((d b) v_{t}\right)^{-1}\right)\right),
\end{gathered}
$$

where $\pi_{\mathcal{S}}^{*}(u d)=s$ and $\pi_{\mathcal{S}_{1}}^{*}((u d))=s_{1}$, for all $d \in D$.

Recall that $\Omega=\left\langle c\left(t_{1} L_{1}, t_{2} L_{1}\right) \mid t_{1}, t_{2} \in \mathcal{T}\right\rangle$ is a subgroup of $\mathcal{O}^{\times}$of order $n$ dividing $m$ so that $(n, p)=1$. Let $\gamma: \Omega \rightarrow \mathcal{O}^{\times}$denote the inclusion linear character (such that $\omega \mapsto \omega$ for all $\omega \in \Omega$ ).

We inflate $c \in Z^{2}\left(H_{1} / L_{1}, \mathcal{O}^{\times}\right)$to an element $\hat{c} \in Z^{2}\left(G, \mathcal{O}^{\times}\right)$, where $\hat{c}\left(k_{1} t_{1}, k_{2} t_{2}\right)$ $=c\left(t_{1} L_{1}, t_{2} L_{1}\right)$ for all $t_{1}, t_{1} \in \mathcal{T}$ and all $k_{1}, k_{1} \in K$.

Using $\hat{c}^{-1} \in Z^{2}\left(G, \mathcal{O}^{\times}\right)$, we construct the group $\hat{G}=\Omega \tilde{\times} G$ as in Lemma 1.2(d), where $|\hat{G}|=n|G|$. Note that

$$
\left(\omega_{1}, k\right)\left(\omega_{2}, g\right)=\left(\omega_{1} \omega_{2}, k g\right), \quad\left(\omega_{2}, g\right)\left(\omega_{1}, k\right)=\left(\omega_{1} \omega_{2}, g k\right)
$$

and

$$
\left(\omega_{2}, g\right)\left(\omega_{1}, k\right)\left(\omega_{2}, g\right)^{-1}=\left(\omega_{1}, g k g^{-1}\right)
$$

for all $\omega_{1}, \omega_{2} \in \Omega$, all $g \in G$ and all $k \in K$ since $\hat{c}(g, h)=1$ if $g \in K$ or $h \in$ $K$. Thus $\hat{K}=\{(1, k) \mid k \in K\}, \hat{L}=\{(1, \ell) \mid \ell \in L\}$ and $\hat{D}=\{(1, d) \mid d \in D\}$ are normal subgroups of $\hat{G}$ and are isomorphic, in the obvious way: $(x \mapsto(1, x))$, to $K, L, D$ respectively. Let $\hat{b}$ denote the image of $b \in Z(\mathcal{O} L)$ in $\mathcal{O} \hat{L}$ under the above isomorphism $L \cong \hat{=} \hat{L}$. Thus $\hat{b}$ is a $\hat{G}$-stable block idempotent of $\mathcal{O} \hat{L}$ and of $\mathcal{O} \hat{K}$ with defect group $Z(\hat{D})$ in $\mathcal{O} \hat{L}$ and defect group $\hat{D}$ in $\mathcal{O} \hat{K}$. Here $C_{\hat{G}}(\hat{D})=i(\Omega) \times \hat{L}$ and $\hat{D} C_{\hat{G}}(\hat{D})=i(\Omega) \times \hat{K}$, where $i$ is described in Lemma $1.2(\mathrm{~d})$.

Let $\hat{\gamma}=\gamma \circ i^{-1}: i(\Omega) \rightarrow \mathcal{O}^{\times}$denote the linear character of $i(\Omega)$ corresponding to $\gamma: \Omega \rightarrow \mathcal{O}^{\times}$and set $\hat{e}=\frac{1}{n} \sum_{\omega \in \Omega} \omega^{-1}(\omega, 1)$ so that $\hat{e}$ is the $\hat{G}$-stable block idempotent of $\mathcal{O} i(\Omega)$ corresponding to $\hat{\gamma}$. Thus $\hat{e} \hat{b}$ is a $\hat{G}$-stable block idempotent of $\mathcal{O}\left(\hat{D} C_{\hat{G}}(\hat{D})\right)$ with defect group $\hat{D}$ and $\hat{e} \hat{b}$ is also a block idempotent of $\mathcal{O} \hat{G}$ with defect group $\hat{D}$.

Let $\hat{\theta} \in \operatorname{Irr}_{\mathcal{K}}(\hat{b})$, where $\hat{b}$ is a block of $\hat{K}$ correspond to $\theta \in \operatorname{Irr}_{\mathcal{K}}(b)$ so that $\hat{D} \leq \operatorname{Ker}(\hat{\theta})$. Clearly $\hat{G}=\bigcup_{t \in \mathcal{T}}(i(\Omega) \times \hat{K})(1, t)$ and the union is disjoint.

Let $\hat{L}_{1}=\left\{\left(1, \ell_{1}\right) \mid \ell_{1} \in L_{1}\right\}$ and $\hat{K}_{1}=\left\{\left(1, k_{1}\right) \mid k_{1} \in K_{1}\right\}$, so that $L_{1} \tilde{=} \hat{L}_{1}$ and $K_{1} \tilde{=} \hat{K}_{1}$ canonically. Also $C_{\hat{K}}(A)=\hat{K}_{1}, C_{\hat{L}}(A)=\hat{L}_{1}$ and $W(\hat{b})$ is a block idempotent of $\mathcal{O} \hat{K}_{1}$ and of $\mathcal{O} \hat{L}_{1}$ with defect groups $\hat{D}, Z(\hat{D})$, respectively.

Since $\hat{c}\left(g_{1}^{a}, g_{2}^{a}\right)=\hat{c}\left(g_{1}, g_{2}\right)$ for all $g_{1}, g_{1} \in G$ and all $a \in A$ by Lemma 2.11, $A$ acts on the right on $\hat{G}$ according to: $(\omega, g)^{a}=\left(\omega, g^{a}\right)$ for all $\omega \in \Omega, g \in G$ and $a \in A$. Here $\hat{D} \leq C_{\hat{G}}(A)=i(\Omega) \times C_{G}(A)$ and $C_{\hat{G}}(A)=\bigcup_{t \in \mathcal{T}}\left(i(\Omega) \times \hat{K}_{1}\right)(1, t)$, where the union is disjoint. Also $C_{\hat{G}}(A) \cap C_{\hat{G}}(\hat{D})=i(\Omega) \times \hat{L}_{1}$ and $\left(\hat{D} C_{\hat{G}}(D)\right) \cap C_{\hat{G}}(A)=$ $i(\Omega) \times \hat{K}_{1}$. Moreover $\widehat{W(b)}$ (the image of $W(b)$ under the isomorphism $L \tilde{=} \hat{L}$ ) is a $C_{\hat{G}}(A)$-stable block of $\mathcal{O} \hat{K}_{1}$ with defect group $\hat{D}, W(\hat{b})=\widehat{W(b)}$ and $\hat{e} \widehat{W(b)}$ is a $C_{\hat{G}}(A)$-stable block idempotent of $i(\Omega) \times \hat{K}_{1}=\hat{D}\left(C_{\hat{G}}(A) \cap C_{\hat{G}}(D)\right)$ with defect group $\hat{D}$. Thus $\hat{e} \widehat{W(b)}$ is a block idempotent of $\mathcal{O} C_{\hat{G}}(A)$ with defect group $\hat{D}$ and $W(\hat{e} \hat{b})$ is also a block idempotent of $\mathcal{O} C_{\hat{G}}(A)$ with defect group $\hat{D}$ by $[5$, V, Lemma 3.10]. Set $\hat{\theta}_{1}=\pi(\hat{K}, A)(\hat{\theta})$ so that $\hat{\theta}_{1} \in \operatorname{Irr}_{\mathcal{K}}(W(\hat{b}))$. 
Lemma 2.18. Let $\hat{\pi}_{\mathcal{S}}^{*}: \hat{G} \rightarrow \mathcal{S}^{\times}$be such that $(\omega, g) \mapsto \omega \pi_{\mathcal{S}}^{*}(g)$ for all $\omega \in \Omega$ and all $g \in G$ and set $\hat{\theta}^{*}=T r_{\mathcal{S}} \circ \hat{\pi}_{\mathcal{S}}^{*}: \hat{G} \rightarrow \mathcal{O}$. Then

(a) $\hat{\pi}_{\mathcal{S}}^{*}$ is a group homomorphism with $\hat{D} \leq \operatorname{Ker}\left(\hat{\pi}_{\mathcal{S}}^{*}\right)=\operatorname{Ker}\left(\widehat{\theta}^{*}\right)$;

(b) $\hat{\pi}_{\mathcal{S}}=\operatorname{Res}_{\hat{K}}^{\hat{G}}\left(\hat{\pi}_{\mathcal{S}}^{*}\right): \hat{K} \rightarrow \mathcal{S}^{\times}$is an irreducible representation of $\hat{K}$ over $\mathcal{K}$ and $\hat{\pi}_{\mathcal{S}}^{*}$ is an irreducible respresentation of $\hat{G}$ over $\mathcal{K}$ with character $\hat{\theta}^{*}$;

(c) $\hat{\theta}^{*}$ lies in the A-stable block $\hat{e} \hat{b}$ of $\mathcal{O} \hat{G}$ and $\hat{\theta}^{*}(\hat{x})=0$ for all $\hat{x} \in \hat{G}$ such that $\hat{x} \hat{D} \notin(\hat{G} / \hat{D})_{p^{\prime}}$;

(d) $\operatorname{Res}_{i(\Omega) \times \hat{K}^{\hat{G}}}\left(\hat{\theta}^{*}\right)=\hat{\gamma} \times \hat{\theta}$ is an A-stable irreducible character that lies in the block $\hat{e} \hat{b}$ of $\mathcal{O}(i(\Omega) \times \hat{K})$ with defect group $\hat{D}$; and

(e) $\pi(\hat{G}, A)\left(\hat{\theta}^{*}\right) \in \operatorname{Irr}_{\mathcal{K}}(W(\hat{e} \hat{b}))$ and $\operatorname{Res}_{i(\Omega) \times \hat{K}}^{C_{\hat{G}}(A)}\left(\pi(\hat{G}, A)\left(\hat{\theta}^{*}\right)\right)=\hat{\gamma} \times \hat{\theta}_{1}$ is an irreducible character that lies in the block $\hat{e} W(\hat{b})$ of $\mathcal{O}\left(i(\Omega) \times \hat{K}_{1}\right)$ with defect group $\hat{D}$.

Proof. Clearly Lemma 2.13 and [5, IV, Lemma 2.4] furnish a proof of (a)-(d). For (e), note that $\operatorname{Res}_{i(\Omega) \times \hat{K}}^{\hat{G}}\left(\hat{\theta}^{*}\right)=\hat{\gamma} \times \hat{\theta}$ and that $\pi(i(\Omega) \times \hat{K}, A)(\hat{\gamma} \times \hat{\theta})=\hat{\gamma} \times \hat{\theta}_{1}$ is an irreducible character that lies in the block $\hat{e} W(\hat{b})$ of $\mathcal{O}\left(i(\Omega) \times \hat{K}_{1}\right)$ since $\pi(K, A)(G)=\theta_{1}$. Now [11, Theorem A(b)] implies that $\operatorname{Res}_{i(\Omega) \times \hat{K}_{1}}^{C_{\hat{G}}(A)}\left(\pi(\hat{G}, A)\left(\hat{\theta}^{*}\right)\right)=$ $\hat{\gamma} \times \hat{\theta}_{1}$ and we are done.

Lemma 2.19. Let $\widehat{\pi_{\mathcal{S}_{1}}^{*}}: C_{\hat{G}}(A) \rightarrow \mathcal{S}_{1}^{\times}$be such that $(\omega, g) \mapsto \omega \pi_{\mathcal{S}_{1}}^{*}(g)$ for all $\omega \in \Omega$ and all $g \in C_{G}(A)$ and set $\widehat{\theta_{1}^{*}}=T r_{\mathcal{S}_{1}} \circ \widehat{\pi_{\mathcal{S}_{1}}^{*}}: C_{\hat{G}}(A) \rightarrow \mathcal{O}$. Then

(a) $\widehat{\pi_{\mathcal{S}_{1}}^{*}}$ is a group homomorphism with $\hat{D} \leq \operatorname{Ker}\left(\widehat{\pi_{\mathcal{S}_{1}}^{*}}\right)=\operatorname{Ker}\left(\widehat{\theta_{1}^{*}}\right)$;

(b) $\operatorname{Res}_{\hat{K}_{1}}^{C_{\hat{G}}(A)}\left(\widehat{\pi_{\mathcal{S}_{1}}^{*}}\right)=\hat{\pi}_{\mathcal{S}_{1}}: \hat{K}_{1} \rightarrow \mathcal{S}_{1}^{\times}$is an irreducible representation of $\hat{K}_{1}$ over $\mathcal{K}$ with character $\hat{\theta}_{1}$ and $\hat{\pi}_{\mathcal{S}_{1}}^{*}$ is an irreducible representation of $C_{\hat{G}}(A)$ over $\mathcal{K}$ with character $\hat{\theta}_{1}^{*}$;

(c) $\operatorname{Res}_{i(\Omega) \times \hat{K}_{1}}^{C_{\hat{G}}(A)}\left(\hat{\theta}_{1}^{*}\right)=\hat{\gamma} \times \hat{\theta}_{1}$;

(d) $\pi(\hat{G}, A)\left(\hat{\theta}^{*}\right)=\lambda \widehat{\theta}_{1}^{*}$ for a unique linear character $\lambda$ of $C_{\hat{G}}(A)$ such that $i(\Omega) \times$ $\hat{K}_{1} \leq \operatorname{Ker}(\lambda)$

(e) $\hat{e} \widehat{W(b)}=W(\hat{e} \hat{b}) ;$ and

(f) $\hat{\theta}_{1}^{*} \in \operatorname{Irr}_{\mathcal{K}}(W(\hat{e} \hat{b}))$ and $\hat{\theta}_{1}^{*}(\hat{x})=0$ for all $\hat{x} \in C_{\hat{G}}(A)$ such that $\hat{x} \hat{D} \notin$ $\left(C_{\hat{G}}(A) / \hat{D}\right)_{p^{\prime}}$.

Proof. Clearly (a)-(c) hold and Lemma 2.18(e) yields (d). We have seen that $\hat{e} W(\hat{b})$ is a block idempotent with defect group $\hat{D}$ of both $\mathcal{O}\left(i(\Omega) \times \hat{K}_{1}\right)$ and $\mathcal{O} C_{\hat{G}}(A)$. Also $W(\hat{e} \hat{b})$ is a block idempotent of $\mathcal{O} C_{\hat{G}}(A)$ with defect group $\hat{D}$ that, by Lemma $2.18(\mathrm{e})$, covers the $C_{\hat{G}}(A)$-stable block $\hat{e} W(\hat{b})$ of $\mathcal{O}\left(i(\Omega) \times \hat{K}_{1}\right)$. Thus (e) holds by [5, V, Lemma 3.10]. By (c), $\hat{\theta}_{1}^{*}$ lies in a block of $\mathcal{O} C_{\hat{G}}(A)$ that covers the block $\hat{e} W(\hat{b})$, and again [5, V, Lemma 3.10] and the proof of Lemma 2.18(c) yield (f) .

Set $\overline{\hat{G}}=\hat{G} / \hat{D}$ and let $-: \hat{G} \rightarrow \overline{\hat{G}}$ denote the canonic group epimorphism. Clearly $A$ induces an action on $\overline{\hat{G}}$ and $-: \overline{\hat{G}} \rightarrow \hat{G} / \hat{D}$ is an $A$-epimorphism. Here $|\overline{\hat{G}}|=n|G / D|, \overline{\hat{G}}=\bigcup_{t \in \mathcal{T}}(\overline{i(\Omega)} \times \overline{\hat{K}}) \overline{(1, t)}$, where the union is disjoint and $C_{\overline{\hat{G}}}(A)=$ $\left.\overline{C_{\hat{G}}(A)}=C_{\hat{G}}(A) / \hat{D}=\bigcup_{t \in \mathcal{T}} \overline{(i(\Omega)} \times \bar{K}_{1}\right) \overline{(1, t)}$, where the union is also disjoint. 
Since $\hat{D} \leq \operatorname{Ker}\left(\hat{\pi}_{\mathcal{S}}^{*}\right)=\operatorname{Ker}\left(\hat{\theta}^{*}\right)$, we can view $\hat{\pi}_{s}^{*}$ and $\hat{\theta}^{*}$ as lying in an $A$-stable block of defect 0 of $\hat{G}$ that is contained in the block $\hat{e} \hat{b}$ of $\hat{G}$ since $\hat{\theta}^{*}(1)=r$, where $r_{p}=|G / D|_{p}=|\hat{G} / \hat{D}|_{p}$. Similar statements hold for $\pi_{\mathcal{S}_{1}}^{*}, \hat{\theta}_{1}^{*}, G_{\overline{\hat{G}}}(A)$ and $C_{\hat{G}}(A)$.

Remark 2.20. Let $(\omega, k t) \in \hat{G}=\Omega \times G$, where $\omega \in \Omega, k \in K, t \in \mathcal{T}$ and let $k t=(k t)_{p}(k t)_{p^{\prime}}$. Then $(k t)_{p} \in K$ since $|G / K|_{p}=1$ and $\left(\omega,(k t)_{p^{\prime}}\right) \in \hat{G}_{p^{\prime}}$. Thus $(\omega, k t)_{p}=\left(1,(k t)_{p}\right),(\omega, k t)_{p^{\prime}}=\left(\omega,(k t)_{p^{\prime}}\right)$. It follows that $(\omega, k t) \hat{D} \in(\overline{\hat{G}})_{p^{\prime}}$ if and only if $(k t) D \in(G / D)_{p^{\prime}}$. A similar result holds for $\left(\omega, k_{1} t\right) \in C_{\hat{G}}(A)=\Omega \times C$, where $\omega \in \Omega, k_{1} \in K_{1}, t \in \mathcal{T}$.

As in Lemma 2.19(d), let $\lambda$ be the unique linear character of $C_{\hat{G}}(A)$ with $i(\Omega) \times$ $\hat{K}_{1} \leq \operatorname{Ker}(\lambda)$ such that $\pi(\hat{G}, A)\left(\hat{\theta}^{*}\right)=\lambda \hat{\theta}_{1}^{*}$. Since $\hat{G}=\hat{K} C_{\hat{G}}(A)$, there is a unique linear character $\hat{\lambda}$ of $\hat{G}$ such that $i(\Omega) \times \hat{K} \leq \operatorname{Ker}(\hat{\lambda})$ and $\operatorname{Res}_{C_{\hat{G}}(A)}^{\hat{\hat{\lambda}}}(\hat{\lambda})=\lambda$. It follows that $\hat{\lambda}^{-1} \hat{\theta}^{*} \in \operatorname{Irr}_{\mathcal{K}}(\hat{e} \hat{b})^{A}$ and $\pi(\hat{G}, A)\left(\hat{\lambda}^{-1} \hat{\theta}^{*}\right)=\hat{\theta}_{1}^{*}$. At this point, for each $t \in \mathcal{T}$, we replace $w_{t}$ by $\hat{\lambda}(t) w_{t}$ and $v_{t}$ by $\hat{\lambda}(t) v_{t}$ in Proposition 2.1. Then, since $w_{t}=s_{t} \alpha_{t}$ where $s_{t} \in \mathcal{S}^{\times}$and $\left.\alpha_{t} \in b+\mathcal{I}(\mathcal{O} L) b\right), s_{t}$ is replaced by $\lambda(t) s_{t}$ for each $t \in \mathcal{T}$. With this replacement, we obtain the new $\mathcal{O}$-linear map $\tilde{\pi}_{\mathcal{S}}^{*}: \mathcal{O} G \rightarrow \mathcal{S}$ sending $k t$ to $\pi_{\mathcal{S}}(k b) \hat{\lambda}(t)^{-1} s_{t}^{-1}$ so that $\operatorname{Tr}_{\mathcal{S}}\left(\pi_{\mathcal{S}}^{*}(k t)\right)=\left(\hat{\lambda}^{-1} \hat{\theta}^{*}\right)(k t)$ for all $k \in K$ and $t \in \mathcal{T}$. Consequently after replacement, we may assume that

$$
\pi(\hat{G}, A)\left(\hat{\theta}^{*}\right)=\hat{\theta}_{1}^{*} .
$$

We have set

$$
\mathcal{B}=C_{(\mathcal{O} G) b}(\mathcal{S})=\oplus_{t \in \mathcal{T}}\left(((\mathcal{O} D) b) v_{t}\right)=\oplus_{\substack{t \in \mathcal{T} \\ d \in D}} \mathcal{O}(d b) v_{t},
$$

so that $\mathcal{B}$ can be viewed as an $\mathcal{N}=D \rtimes\left(H_{1} / L_{1}\right)$-twisted group $\mathcal{O}$-algebra with $\mathcal{O}$-bases $\left\{(d b) v_{t} \mid d \in D, t L_{1} \in H_{1} / L_{1}\right\}$, where

$$
\left(\left(d_{1} b\right) v_{t_{1}}\right)\left(\left(d_{2} b\right) v_{t_{2}}\right)=c\left(t_{1} L_{1}, t_{2} L_{1}\right)\left(d_{1}\left({ }^{t_{1}} d_{2}\right)\right) v_{t_{3}}
$$

if $t_{1} t_{2} \in t_{3} L_{1}$ for a unique $t_{3} \in \mathcal{T}$ and $\mathcal{B}_{\left(d, t L_{1}\right)}=\mathcal{O}(d b) v_{t}$ for all $d \in D$ and $t \in \mathcal{T}$. Also we have set $\mathcal{B}^{\prime}=C_{(\mathcal{O C}) W(b)}\left(\mathcal{S}_{1}\right)=\oplus_{t \in \mathcal{T}}\left(((\mathcal{O} D) W(b)) v_{t}^{\prime}\right)$ and we view $\mathcal{B}^{\prime}$ as an $\mathcal{N}=D \rtimes\left(H_{1} / L_{1}\right)$-twisted group $\mathcal{O}$-algebra, where $\mathcal{B}_{\left(d, t L_{1}\right)}^{\prime}=\mathcal{O}(d W(b)) v_{t}^{\prime}$ for all $d \in D$ and $t \in \mathcal{T}$. Thus $\Phi: \mathcal{B}^{\prime} \rightarrow \mathcal{B}$ is an $\mathcal{N}$-graded $\mathcal{O}$-algebra isomorphism sending $(d W(b)) v_{t}^{\prime}$ to $(d b) v_{t}$ for all $d \in D$ and $t \in \mathcal{T}$.

We inflate $c \in Z^{2}\left(H_{1} / L_{1}, \mathcal{O}^{\times}\right)$to an element $\tilde{c} \in Z^{2}\left(\mathcal{N}, \mathcal{O}^{\times}\right)$, where

$$
\tilde{c}\left(\left(d_{1}, t_{1} L_{1}\right),\left(d_{2}, t_{2} L_{1}\right)\right)=c\left(t_{1} L_{1}, t_{2} L_{2}\right)
$$

for all $d_{1}, d_{2} \in D$ and all $t_{1}, t_{2} \in \mathcal{T}$. Then, using $\tilde{c}$ and Lemma 1.2, we obtain a finite group $\tilde{\mathcal{N}}=\Omega \tilde{\times} \mathcal{N}$, where $|\tilde{\mathcal{N}}|=n|D| m$ and we let $A$ act trivially on $\tilde{\mathcal{N}}$. Here $\tilde{D}=\left\{\left(1,\left(d, L_{1}\right)\right) \mid d \in D\right\}$ is a normal Sylow $p$-subgroup of $\tilde{\mathcal{N}}, D \tilde{D} \tilde{D}$ via the map $d \mapsto\left(1,\left(d, L_{1}\right)\right)$ for all $d \in D,\left\{\left(\omega,\left(1, t L_{1}\right)\right) \mid \omega \in \Omega, t \in \mathcal{T}\right\}$ is a complement to $D$ and $\tilde{\mathcal{N}}=\bigcup_{t \in \mathcal{T}}(i(\Omega) \times \tilde{D})\left(1,\left(1, t L_{1}\right)\right)$, where the union is disjoint.

Set $\tilde{e}=\frac{1}{n} \sum \omega^{-1}\left(\omega,\left(1, L_{1}\right)\right) \in \mathcal{O} i(\Omega)$, so that $\left(\omega,\left(d, t L_{1}\right)\right) \tilde{e}=\omega\left(1,\left(d, t L_{1}\right)\right) \tilde{e}$ for all $\omega \in \Omega, d \in D$ and $t \in \mathcal{T}$. Then $\tilde{e}$ is an $\tilde{\mathcal{N}}$-stable block idempotent of $\mathcal{O} i(\Omega)$ (corresponding to $\left.\tilde{\gamma}=\gamma \circ i^{-1}\right), \tilde{e} \in Z(\mathcal{O} \tilde{\mathcal{N}}),(\mathcal{O} \tilde{\mathcal{N}}) \tilde{e}=\underset{\substack{\oplus_{t \in \mathcal{T}} \\ \mathcal{O}}}{ }\left(1,\left(d, t L_{1}\right)\right) \tilde{e}$ in $\mathcal{O}$-mod and the $\mathcal{O}$-linear map

$$
\Psi:(\mathcal{O} \tilde{\mathcal{N}}) \tilde{e} \rightarrow \mathcal{B}^{\prime}=\underset{\substack{d \in \mathcal{T} \\ t \in \mathcal{T}}}{\mathcal{O}}\left((d W(b)) v_{t}^{\prime}\right)
$$


such that $\left(1,\left(d, t L_{1}\right)\right) \tilde{e} \mapsto(d W(b)) v_{t}^{\prime}$ for all $d \in D$ and $\in \mathcal{T}$ is an $\mathcal{O}$-algebra isomorphism.

Let $A$ act diagonally on the right on $\overline{\hat{G}} \times \tilde{\mathcal{N}}$ so that $C_{\overline{\hat{G}} \times \mathcal{N}}(A)=C_{\overline{\hat{G}}}(A) \times \tilde{\mathcal{N}}$. Set $\Delta=\left\{\left(\overline{(\omega, k t)},\left(\omega^{-1},\left(d, t L_{1}\right)\right)\right) \mid \omega \in \Omega, d \in D, k \in K\right.$ and $\left.t \in \mathcal{T}\right\}$.

The following result is easily verified:

Lemma 2.21. (a) $\Delta$ is an A-invariant subgroup of $\overline{\hat{G}} \times \tilde{\mathcal{N}}$ with $|\Delta|=n|G|$ and $C_{\Delta}(A)=\left\{\left(\overline{\left(\omega, k_{1} t\right)},\left(\omega^{-1},\left(d, t L_{1}\right)\right)\right) \mid \omega \in \Omega, d \in D, t \in \mathcal{T}\right.$ and $\left.k_{1} \in K_{1}\right\} ;$

(b) $(i(\Omega) \times i(\Omega)) \cap \Delta=\left\{\left(\overline{(\omega, 1)},\left(\omega^{-1},\left(1, L_{1}\right)\right)\right) \mid \omega \in \Omega\right\} \leq Z(\Delta) \cap C_{\Delta}(A)$ and the map of $\Omega \rightarrow(i(\Omega) \times i(\Omega)) \cap \Delta$ such that $\left.\omega \mapsto \overline{(\omega, 1)},\left(\omega^{-1},\left(1, L_{1}\right)\right)\right)$ for all $\omega \in \Omega$ is an isomorphism; and

(c) $\mathcal{D}=\left\{\left(\overline{(1,1)},\left(1,\left(d, L_{1}\right)\right)\right) \mid d \in D\right\} \leq \Delta$ and the map of $D \rightarrow \mathcal{D}$ such that $\left.d \mapsto \overline{(1,1)},\left(1,\left(d, L_{1}\right)\right)\right)$ for all $d \in D$ is an isomorphism.

Recall the $\mathcal{O}$-algebra isomorphism $\Phi: \mathcal{B}^{\prime} \rightarrow \mathcal{B}$ and $\Psi:(\mathcal{O} \tilde{\mathcal{N}}) \tilde{e} \rightarrow \mathcal{B}^{\prime}$. Thus $\operatorname{Irr}_{\mathcal{K}}((\mathcal{K} \tilde{\mathcal{N}}) \tilde{e})=\left\{\psi \Phi \Psi \mid \psi \in \operatorname{Irr}_{\mathcal{K}}(\mathcal{K} \mathcal{B})\right\}$. Also $\hat{\theta}^{*}=\operatorname{Tr}_{\mathcal{S}} \circ \hat{\pi}_{\mathcal{S}}^{*} \in \operatorname{Irr}_{\mathcal{K}}(\hat{G})$ and $\hat{\theta}_{1}^{*}=$ $\operatorname{Tr}_{\mathcal{S}_{1}} \circ \hat{\pi}_{\mathcal{S}_{1}}^{*} \in \operatorname{Irr}_{\mathcal{K}}\left(C_{\hat{G}}(A)\right)$. Let $\overline{\hat{\theta}}^{*}$ and $\overline{\hat{\theta}}_{1}^{*}$ denote the irreducible characters of $\overline{\hat{G}}=\hat{G} / \hat{D}$ and of $C_{\overline{\hat{G}}}(A)=C_{\hat{G}}(A) / \hat{D}$ from which $\hat{\theta}^{*}$ and $\hat{\theta}_{1}^{*}$ are inflated, respectively. Thus $\overline{\hat{\theta}}^{*} \times(\psi \Phi \Psi) \in \operatorname{Irr}_{\mathcal{K}}(\overline{\hat{G}} \times \tilde{\mathcal{N}})$ and $\overline{\hat{\theta}}_{1}^{*} \times(\psi \Phi \Psi) \in \operatorname{Irr}_{\mathcal{K}}\left(C_{\overline{\hat{G}} \times \tilde{\mathcal{N}}}(A)\right)$ and $\Delta \cap i(\Omega) \leq$ $\operatorname{Ker}\left(\overline{\hat{\theta}}^{*} \times(\psi \Phi \Psi)\right) \cap \operatorname{Ker}\left(\overline{\hat{\theta}}_{1}^{*} \times(\psi \Phi \Psi)\right)$ for all $\psi \in \operatorname{Irr}_{\mathcal{K}}(\mathcal{K} \mathcal{B})$.

Let $\omega \in \Omega, t \in \mathcal{T}, d \in D, k \in K$ and $k_{1} \in K_{1}$ and $\psi \in \operatorname{Irr}_{\mathcal{K}}(\mathcal{B})$. Note that $\overline{(\omega, k t)} \in \overline{\hat{G}}_{p^{\prime}}$ if and only if $k_{1} t D \in(G / D)_{p^{\prime}}$ and $\overline{\left(\omega, k_{1} t\right)} \in C_{\overline{\hat{G}}}(A)_{p^{\prime}}$ if and only if $k_{1} t D \in\left(C_{G}(A) / D\right)_{p^{\prime}}$ by Remark 2.20. Also $\left.\overline{(\omega, k t)},\left(\omega^{-1},\left(d, t L_{1}\right)\right)\right) \in \Delta$ and $\left.\overline{\left(\left(\omega, k_{1} t\right)\right.},\left(\omega^{-1},\left(d, t L_{1}\right)\right)\right) \in C_{\Delta}(A)$.

Thus we have

$$
\begin{gathered}
\left.\left(\overline{\hat{\theta}}^{*} \times(\psi \Phi \Psi)\right) \overline{(\overline{(\omega, k t)},}\left(\omega^{-1},\left(d, t L_{1}\right)\right)\right) \\
= \begin{cases}0 & \text { if } k t D \notin(G / D)_{p^{\prime}}, \\
\left(T r_{\mathcal{S}^{\circ}} \pi_{\mathcal{S}}^{*}\right)((k t) b) \psi\left((d b) v_{t}\right) & \text { if } k t D \in(G / D)_{p^{\prime}} ;\end{cases}
\end{gathered}
$$

and

$$
\begin{gathered}
\left(\overline{\hat{\theta}}_{1}^{*} \times(\psi \Phi \Psi)\right) \overline{\left(\overline{\left(\omega, k_{1} t\right)},\left(\omega^{-1},\left(d, t L_{1}\right)\right)\right)} \\
= \begin{cases}0 & \text { if } k_{1} t D \notin(C / D)_{p^{\prime}}, \\
\left(\operatorname{Tr}_{\mathcal{S}_{1}^{o}} \pi_{\mathcal{S}_{1}}^{*}\right)\left(\left(k_{1} t\right) W(b)\right) \psi\left((d b) v_{t}\right) & \text { if } k_{1} t D \in(C / D) p^{\prime} .\end{cases}
\end{gathered}
$$

Consequently

(2.11) if $(k t) D \in(G / D)_{p^{\prime}}$, then

$$
\begin{gathered}
\sum_{d \in D}\left(\left(\overline{\hat{\theta}}^{*} \times(\psi \Phi \Psi)\right) \overline{((\omega, k t)},\left(\omega^{-1},\left(d, t L_{1}\right)\right)\right) \\
\cdot\left(\left(\overline{\hat{\theta}}^{*} \times \psi \Phi \Psi\right)\left(\left(\overline{(\omega, k t)},\left(\omega^{-1}, d t L_{1}\right)\right)^{-1}\right)\right) \\
=\left(\left(\operatorname{Tr}_{\mathcal{S}^{\circ}} \pi_{\mathcal{S}}^{*}\right)((k t) b)\right)\left(\left(\operatorname{Tr}_{\mathcal{S}^{\circ}} \pi_{\mathcal{S}}^{*}\right)\left(((k t) b)^{-1}\right)\right) \\
\cdot\left|D: C_{D}(t)\right| \sum_{d \in C_{D}(t)}\left(\psi\left((d b) v_{t}\right)\right)\left(\psi\left(\left((d b) v_{t}\right)^{-1}\right)\right) ;
\end{gathered}
$$


(2.12) if $k_{1} t D \in(C / D)_{p^{\prime}}$, then

$$
\begin{aligned}
& \sum_{d \in D}\left(\left(\overline{\hat{\theta}}_{1}^{*} \times \psi \Phi \Psi\right)\left(\overline{\left(\omega, k_{1} t\right)},\left(\omega^{-1},\left(d, t L_{1}\right)\right)\right)\right. \\
& \left.\cdot\left(\left(\overline{\hat{\theta}}_{1}^{*} \times \psi \Phi \Psi\right) \overline{\left(\omega, k_{1} t\right)},\left(\omega^{-1},\left(d, t L_{1}\right)\right)^{-1}\right)\right) \\
& =\left(\left(\operatorname{Tr}_{\mathcal{S}_{1}^{\circ}} \pi_{\mathcal{S}_{1}}^{*}\right)\left(\left(k_{1} t\right) w(b)\right)\right)\left(\left(\operatorname{Tr}_{\mathcal{S}_{1}^{0}} \pi_{\mathcal{S}_{1}}^{*}\right)\left(\left(\left(k_{1} t\right) W(b)\right)^{-1}\right)\right) \\
& \cdot\left|D: C_{D}(t)\right| \sum_{d \in C_{D}(t)}\left(\psi(d b) v_{t}\right)\left(\psi\left(\left((d b) v_{t}\right)^{-1}\right)\right) ;
\end{aligned}
$$

and

$$
\begin{aligned}
\sum_{d \in D}\left(\left(\overline{\hat{\theta}}^{*} \times(\psi \Phi \Psi)\right) \overline{\left(\omega, k_{1} t\right)},\left(\omega^{-1},\left(d, t L_{1}\right)\right)\right) \\
\left.\cdot\left(\left(\overline{\hat{\theta}}_{1}^{*} \times \psi \Phi \Psi\right)\right)\left(\overline{\left(\left(\omega, k_{1} t\right)\right.},\left(\omega^{-1},\left(d, t L_{1}\right)\right)^{-1}\right)\right) \\
=\left(\left(T r_{\mathcal{S}^{0}} \pi_{\mathcal{S}}^{*}\right)\left(\left(k_{1} t\right) b\right)\right)\left(\left(T r_{\mathcal{S}_{1}^{0}} \pi_{\mathcal{S}_{1}}\right)\left(\left(\left(k_{1} t\right) W(b)\right)^{-1}\right)\right) \\
\cdot\left|D: C_{D}(t)\right| \sum_{d \in C_{D}(t)}\left(\psi\left((d b) v_{t}\right)\right)\left(\psi\left(\left((d b) v_{t}\right)^{-1}\right)\right) .
\end{aligned}
$$

Now compare (2.3), (2.4), (2.5), (2.6), (2.9), (2.10), (2.11) and (2.12).

Thus we conclude that $\left(\operatorname{Res}_{\Delta}^{\overline{\hat{G}} \times \tilde{\mathcal{N}}}\left(\overline{\hat{\theta}}^{*} \times(\psi \Phi \Psi)\right), \operatorname{Res}_{\Delta}^{\hat{G} \times \mathcal{N}}\left(\overline{\hat{\theta}}^{*} \times(\psi \Phi \Psi)\right)\right)_{\Delta}=1$ so that $\operatorname{Res}_{\Delta}^{\overline{\hat{G}} \times \tilde{\mathcal{N}}}\left(\overline{\hat{\theta}}^{*} \times(\psi \Phi \Psi)\right) \in \operatorname{Irr}_{\mathcal{K}}(\Delta)$. Similarly $\operatorname{Res}_{C_{\Delta}}^{C_{\overline{\hat{G}}}(A) \times \tilde{\mathcal{N}}}\left(\overline{\hat{\theta}}_{1}^{*} \times(\psi \Phi \Psi)\right) \in$ $\operatorname{Irr}_{\mathcal{K}}\left(C_{\Delta}(A)\right)$.

Since we have assured that $\pi(\hat{G}, A)\left(\hat{\theta}^{*}\right)=\hat{\theta}_{1}^{*}$, we conclude that $\pi(\overline{\hat{G}}, A)\left(\overline{\hat{\theta}}^{*}\right)=\overline{\hat{\theta}}_{1}^{*}$. Then [11, Theorem A(b)] (with $H=\Delta$ ) implies that

$$
\begin{aligned}
\pi(\Delta, A)\left(\operatorname{Res}_{\Delta}^{\overline{\hat{G}} \times \tilde{\mathcal{N}}}\left(\overline{\hat{\theta}}^{*} \times(\psi \Phi \Psi)\right)\right) & =\operatorname{Res}_{C_{\Delta}(A)}^{C_{\bar{G}}(A) \times \tilde{\mathcal{N}}}\left(\pi(\overline{\hat{G}} \times \tilde{\mathcal{N}}, A)\left(\overline{\hat{\theta}}^{*} \times(\psi \Phi \Psi)\right)\right) \\
& =\operatorname{Res}_{C_{\Delta}(A)}^{C_{\overline{\tilde{G}}}(A) \times \tilde{\mathcal{N}}}\left(\overline{\hat{\theta}}_{1}^{*} \times(\psi \Phi \Psi)\right) .
\end{aligned}
$$

Hence $\rho=\left(\operatorname{Res}_{C_{\Delta}(A)}^{\overline{\hat{G}} \times \tilde{\mathcal{N}}}\left(\overline{\hat{\theta}}^{*} \times(\psi \Phi \Psi)\right), \operatorname{Res}_{C_{\Delta}(A)}^{C_{\overline{\hat{G}}}(A) \times \tilde{\mathcal{N}}}\left(\overline{\hat{\theta}}_{1}^{*} \times(\psi \Phi \Psi)\right)\right)_{C_{\Delta}(A)}$ is relatively prime to $q$. However (2.7) and (2.13) imply that $\rho=\left(\operatorname{Res}_{C}^{G}\left(\theta_{\psi}\right), \theta_{1 \psi}\right)_{C}$. Consequently $\pi(G, A)\left(\theta_{\psi}\right)=\theta_{1 \psi}$ by [10, Theorem 13.1(c)] which concludes our proof of Theorem 2 .

\section{REFERENCES}

1. R. Brauer, zur Darstellungstheorie der Gruppen endlicher Ordnung I, Math. Zeit. 63 (1956), 406-444. MR 17:824g

2. R. Brauer, zur Darstellungstheorie der Gruppen endlicher Ordnung. II, Math. Zeit. 72 (1959), 25-46. MR 21:7258

3. E.C. Dade, Isomorphisms of Clifford extensions, Ann. of Math. 92 (1970), 375-433. MR 42:4645

4. E.C. Dade, Group-graded rings and modules, Math. Z. 174 (1980), 241-262. MR 82c:16028

5. W. Feit, "The Representation Theory of Finite Groups", North-Holland, New York, 1982. MR 83g:20001

6. G. Glauberman, Correspondence of characters for relatively prime operator groups, Canad. J. Math. 20 (1968), 1465-1488. MR 38:1189

7. D. Gorenstein, "Finite Groups", Harper and Row, New York, 1968. MR 38:229

8. M.E. Harris and M. Linckelmann, On the Glauberman and Watanabe correspondence for blocks of finite $p$-solvable groups, Trans. of the A.M.S. 354 (9) (2002), 3435-3453. MR 2003c:20008 
9. B. Huppert, "Endliche Gruppen I", Springer-Verlag, Berlin, 1967. MR 37:302

10. I.M. Isaacs, "Character Theory of Finite Groups", Academic Press, New York, 1976. MR 57:417

11. I.M. Isaacs and G. Navarro, Character correspondences and irreducible induction and restriction, J. of Algebra 140 (1991), 131-140. MR 93a:20015

12. R. Knörr, Blocks, vertices and normal subgroups, Math. Z. 148 (1976), 53-60. MR 53:5723

13. S. Koshitani, personal communication.

14. S. Koshitani and G.O. Michler, Glauberman Correspondence of $p$-Blocks of Finite Groups, J. of Algebra 243 (2001), 504-517. MR 2002g:20024

15. B. Külshammer, Crossed products and blocks with normal defect groups, Comm. Algebra 13 (1985), 147-168. MR 86c:20015

16. W.F. Reynolds, Blocks and normal subgroups, Nagoya Math. J. 22 (1963), 15-32. MR 27:3690

17. J.-P. Serre, "Corps locaux", Hermann, Paris, 1962.

18. J. Thevenaz, "G-Algebras and Modular Representation Theory", Oxford University Press, New York, 1995. MR 96j:20017

19. A. Watanabe, The Glauberman character correspondence and perfect isometries for blocks of finite groups, J. Algebra (1999), 548-565. MR 2000f:20015

School of Mathematics, University of Minnesota, Minneapolis, Minnesota 55455

E-mail address: harris@math.umn.edu 